\title{
Equivalence of three wall-crossing formulæ
}

\author{
ASHOKE SEN
}

\begin{abstract}
The wall-crossing formula of Kontsevich and Soibelman gives an implicit relation between the BPS indices on two sides of the wall of marginal stability by equating two symplectomorphisms constructed from the indices on two sides of the wall. The wall-crossing formulæ of Manschot, Pioline and the author give two apparently different explicit expressions for the BPS index on one side of the wall in terms of the BPS indices on the other side. We prove the equivalence of all the three formulæ.
\end{abstract}

1. Introduction

2. "Higgs branch" wall-crossing formula

3. KS wall-crossing formula

4. Recursion relations for the KS wall-crossing formula

5. Equivalence of KS and "Hhiggs branch" wall-crossing formulæ

\subsection{Comparison with the constraints on $\sigma$} for non-vanishing $\mathrm{N}_{\text {higgs }}^{(n+1)}\left(\left\{\alpha_{i}\right\} ; \sigma\right)$

5.5. Proof of $\mathrm{N}_{\mathrm{KS}}^{(n+1)}=\mathrm{N}_{\text {higgs }}^{(n+1)}$

6. An alternative approach to proving the equivalence of the Higgs branch and KS wall-crossing formulæ 


\section{Equivalence of "Higgs" and "Coulomb" branch wall-crossing formulæ}

\section{References}

\section{Introduction}

The central objects in a wall-crossing formula are a BPS index in some Hilbert space $\mathcal{H}$, and a moduli space over which the Hilbert space could vary. The BPS index remains constant over most of the moduli space but could jump across certain codimension one subspaces known as the walls of marginal stability. When this happens, wall-crossing formula gives a relation between the BPS indices on two sides of the wall [1-39].

In situations relevant to supersymmetric string theory/gauge theory the Hilbert space that is of relevance is the space of quantum states carrying some fixed set of gauge charges, collectively denoted by a vector $\gamma$. The moduli space is parametrized by the asymptotic values of certain scalar fields of the theory. If the theory also contains some global conserved $U(1)$ charge $Q$, then we can define a refined index $\Omega_{\text {ref }}(\gamma, y)$, which computes the index weighted by $y^{Q}$ for some continuous variable $y$. Under certain circumstances this refined index also remains constant over most of the moduli space and jumps only across the walls of marginal stability. For example in supersymmetric gauge theories we can define such an index by taking $Q$ to be an appropriate linear combination of the angular momentum and R-symmetry generator [27]. In string theory, there are no global R-symmetry charges, but we can define a refined index by taking $Q$ to be one of the angular momentum generators [40,41]. Such an index is not protected under a change in the string coupling, i.e., it can jump even without crossing a wall of marginal stability, but we could nevertheless study its jump across the walls of marginal stability keeping the string coupling fixed at some small value. A refined wall-crossing formula is a relation between the refined indices on two sides of the wall of marginal stability. This is more general than ordinary (also referred to as "numerical") wall-crossing formula, since by setting $y=1$ in the former we recover the latter. 
The known wall-crossing formulæ take simpler form in terms of the "rational refined index" defined as [14-16, 42-44]

$$
\bar{\Omega}_{\mathrm{ref}}(\gamma, y) \equiv \sum_{m \mid \gamma} \frac{y-y^{-1}}{m\left(y^{m}-y^{-m}\right)} \Omega_{\mathrm{ref}}\left(\gamma / m, y^{m}\right)
$$

In the $y \rightarrow 1$ limit this gives $\bar{\Omega}(\gamma)=\sum_{m \mid \gamma} m^{-2} \Omega(\gamma / m)$. We shall denote by $\Omega_{\text {ref }}^{ \pm}(\gamma, y)$ the refined indices on two sides of a wall of marginal stability, and by $\bar{\Omega}_{\text {ref }}^{ \pm}(\gamma, y)$ the corresponding rational refined indices. A wall-crossing formula corresponds to a relation between $\Omega_{\text {ref }}^{+}$and $\Omega_{\text {ref }}^{-}$, or equivalently between $\bar{\Omega}_{\text {ref }}^{+}$and $\bar{\Omega}_{\text {ref }}^{-}$. We shall work with the indices $\bar{\Omega}_{\text {ref }}^{ \pm}$but if needed we can invert (1.1) to calculate $\Omega_{\text {ref }}^{ \pm}$in terms of $\bar{\Omega}_{\text {ref }}^{ \pm}[30,33]$.

The charge $\gamma$ is a member of some charge lattice equipped with a symplectic inner product. Given a pair of vectors $\gamma_{1}, \gamma_{2}$ on the charge lattice, we denote by $\left\langle\gamma_{1}, \gamma_{2}\right\rangle$ the symplectic inner product between $\gamma_{1}$ and $\gamma_{2}$. This inner product is anti-symmetric under the exchange of $\gamma_{1}$ and $\gamma_{2}$ and is linear in $\gamma_{1}, \gamma_{2}$. Typically, the charge lattice has dimension $d$ for some even integer $d$, but for a given wall of marginal stability the relevant charge vectors for which the index jumps across the wall are of the form $r \alpha+s \beta$, where $\alpha, \beta$ are two vectors whose central charges align at the wall and $r$ and $s$ are two non-negative rational numbers. We denote by $\Lambda$ the set of all such non-zero charge vectors in the lattice. Without any loss of generality we can choose a convention in which $\langle\beta, \alpha\rangle>0$ and represent $r \alpha+s \beta \in \Lambda$ by a vector $(r, s)$ in a two-dimensional (2D) plane. In this convention, all the elements of $\Lambda$ are represented as vectors in the first quadrant of this $2 \mathrm{D}$ plane, and given $\gamma_{1}, \gamma_{2} \in \Lambda, \gamma_{1}$ and $\gamma_{2}$ are arranged in a clockwise (anti-clockwise) order if $\left\langle\gamma_{1}, \gamma_{2}\right\rangle>0\left(\left\langle\gamma_{1}, \gamma_{2}\right\rangle<0\right)$ (see figure 1$)$. We denote by $Z_{\gamma}$ the central charge for any vector $\gamma \in \Lambda$ - a function of the moduli and a linear function of $\gamma$ such that the mass of a BPS state of charge $\gamma$ is given by $\left|Z_{\gamma}\right|$ - and choose our convention such that

$$
\left\langle\gamma_{1}, \gamma_{2}\right\rangle \operatorname{Im}\left(Z_{\gamma_{1}} \bar{Z}_{\gamma_{2}}\right)<0, \quad \forall \gamma_{1}, \gamma_{2} \in \Lambda
$$

on the side of the wall in which we label the index by $\Omega_{\text {ref }}^{+}$. On the other side of the wall $\left\langle\gamma_{1}, \gamma_{2}\right\rangle \operatorname{Im}\left(Z_{\gamma_{1}} \bar{Z}_{\gamma_{2}}\right)>0$ and the refined index is denoted by $\Omega_{\mathrm{ref}}^{-}$. Then the wall-crossing formula, written in the notation of [30], takes the form:

$$
\bar{\Omega}_{\text {ref }}^{-}(\gamma, y)=\sum_{n \geq 1} \sum_{\begin{array}{c}
\text { unordered set } \alpha_{1}, \ldots, \alpha_{n} \in \Lambda \\
\alpha_{1}+\cdots+\alpha_{n}=\gamma
\end{array}} \frac{g_{\text {ref }}\left(\left\{\alpha_{i}\right\}, y\right)}{\left|\operatorname{Aut}\left(\left\{\alpha_{i}\right\}\right)\right|} \prod_{i=1}^{n} \bar{\Omega}_{\text {ref }}^{+}\left(\alpha_{i}, y\right),
$$


where $g_{\mathrm{ref}}\left(\left\{\alpha_{i}\right\}, y\right)$ is a function to be specified later, and $\left|\operatorname{Aut}\left(\left\{\alpha_{i}\right\}\right)\right|$ is a symmetry factor defined as follows. If the set $\left\{\alpha_{i}\right\}$ consists of $m_{1}$ copies of $\beta_{1}, m_{2}$ copies of $\beta_{2}$ etc. then $\left|\operatorname{Aut}\left(\left\{\alpha_{i}\right\}\right)\right|=\prod_{k} m_{k}$ !. The sum in (1.3) runs over all possible unordered decompositions of the vector $\gamma$ into the vectors $\alpha_{1}, \ldots, \alpha_{n} \in \Lambda$. However this can also be rewritten as a sum over the ordered decomposition of the vector $\gamma$ into the vectors $\alpha_{1}, \ldots, \alpha_{n} \in \Lambda$ as follows:

$$
\begin{aligned}
\bar{\Omega}_{\mathrm{ref}}^{-}(\gamma, y)= & \sum_{n \geq 1} \frac{1}{n !} \sum_{\text {ordered decomposition } \alpha_{1}+\cdots+\alpha_{n}=\gamma} g_{\mathrm{ref}}\left(\left\{\alpha_{i}\right\}, y\right) \\
& \times \prod_{i=1}^{n} \bar{\Omega}_{\mathrm{ref}}^{+}\left(\alpha_{i}, y\right) .
\end{aligned}
$$

For a single argument $g_{\text {ref }}(\alpha ; y)$ is taken to be 1 , so that the $n=1$ term on the right-hand side of (1.3) just gives $\bar{\Omega}_{\text {ref }}^{+}(\gamma, y)$. The wall-crossing formula for rational numerical index can be found by taking the $y \rightarrow 1$ limit of the above formula.

One of the results discussed in [30] is that once we use the index $\bar{\Omega}$ instead of $\Omega$, the effect of having two or more identical $\alpha_{i}$ 's is captured completely by the symmetry factor $\left|\operatorname{Aut}\left(\left\{\alpha_{i}\right\}\right)\right|=\prod_{k} m_{k}$ !. In order to make full utilization of this fact, it is useful to regard the $\alpha_{i}$ 's as elements of a $2 D$ vector space spanned by $\alpha$ and $\beta$, not necessarily lying on the lattice, and $g_{\text {ref }}$ as continuous function of these $\alpha_{i}$ 's. We shall give the expressions for $g_{\text {ref }}$ for generic non-identical, non-parallel vectors $\alpha_{i}$ lying in the first quadrant of the $2 \mathrm{D}$ plane spanned by $\alpha, \beta$. From this, we can recover the results for two or more identical or parallel $\alpha_{i}$ 's as limits of this general formula. ${ }^{1}$

The wall-crossing formula of Kontsevich and Soibelman (KS) [11-13] and Manschot, Pioline and the author (MPS) [30] differ in their specification of the functions $g_{\text {ref }}$. Manschot et al. [30] actually proposed two different versions of the wall-crossing formula. The first one, called the "Higgs branch formula", is based on Reineke's result on quiver moduli spaces [45] (see also $[14,46]$ for related results), and the second one, called the "coulomb branch formula", is based on quantum mechanics of multiple black holes [7-10]. We shall describe the Higgs branch formula for $g_{\text {ref }}$ in Section 2 and the KS formula for $g_{\text {ref }}$ in Section 3. In either case, we shall describe the

\footnotetext{
${ }^{1}$ For the KS wall-crossing formula, this prescription was proved in [30] (last paragraph of Section 4.4). For the MPS wall-crossing formulæ this is included in the prescription for computing $g_{\text {ref }}$, and follows from the ability to replace Bose/Fermi statistics by Boltzmann statistics at the cost of replacing $\Omega$ by $\bar{\Omega}$. To our knowledge this has not been proved for the Joyce-Song (JS) wall-crossing formula [14-16], but the agreement between JS and other wall-crossing formulæ in explicit examples indicate that this is valid also in that case.
} 


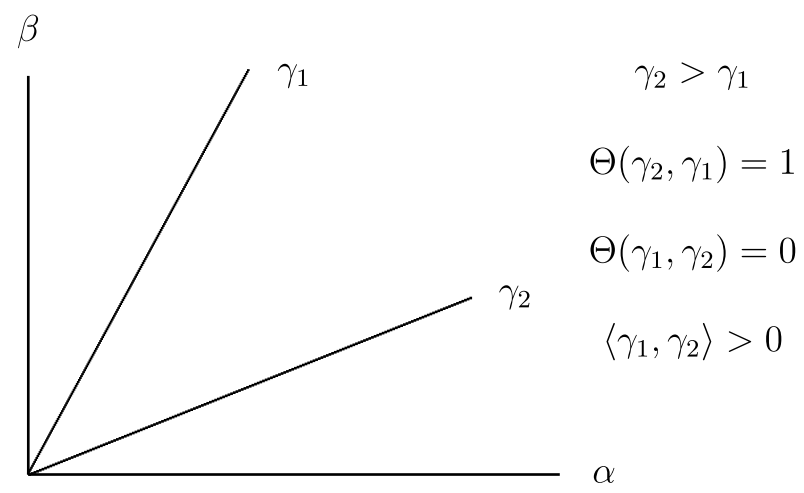

Figure 1: Figure illustrating the definition of $\gamma_{2}>\gamma_{1}$ and $\Theta\left(\gamma_{2}, \gamma_{1}\right)$.

formula as a function of generic non-identical, non-parallel vectors $\alpha_{i}$ in the first quadrant of the plane spanned by $\alpha$ and $\beta$. The equivalence of the two formulæ was tested in [30] for low values of $n$ but was not proven. In Sections 4 and 5, we prove the equality of these two apparently different formulæ for $g_{\text {ref }}$. Finally in Section 7, we describe the coulomb branch formula for $g_{\text {ref }}$ and prove its equality with the Higgs branch formula.

Since our Higgs branch formula is based on Reineke's formula on quiver moduli spaces [45] and since the latter has close relationship with both the wall-crossing formulæ of $\mathrm{KS}$ [11] as well as that of Joyce and Song [14-16], the equality of the Higgs branch formula and the KS formula is not unexpected [47]. Nevertheless our analysis gives a direct combinatorial proof of this equivalence. The equivalence with the coulomb branch formula is new, - to our knowledge this has not appeared in connection with the wall-crossing formula before [30].

Notations and conventions: We shall end this section by describing some useful notations and conventions, which we shall use. We define:

$$
\begin{aligned}
& \gamma_{1}<\gamma_{2} \text { if }\left\langle\gamma_{1}, \gamma_{2}\right\rangle>0, \\
& \gamma_{1}>\gamma_{2} \text { if }\left\langle\gamma_{1}, \gamma_{2}\right\rangle<0 \\
& \Theta\left(\gamma_{1}, \gamma_{2}\right)= \begin{cases}1 & \text { for } \gamma_{1}>\gamma_{2} \\
0 & \text { for } \gamma_{1}<\gamma_{2}\end{cases}
\end{aligned}
$$

Thus for example if $\left(\gamma_{1}, \gamma_{2}\right)$ follows a clockwise order then $\gamma_{1}<\gamma_{2}$ and $\Theta\left(\gamma_{2}, \gamma_{1}\right)=1, \Theta\left(\gamma_{1}, \gamma_{2}\right)=0$. Since this notation will be used extensively in the rest of the paper, it will be useful to keep in mind the physical picture shown in figure 1 . We shall also sometimes describe the situation in figure 1 
by saying that $\gamma_{1}$ is to the left of $\gamma_{2}$ or that $\gamma_{2}$ is to the right of $\gamma_{1}$. (1.5) satisfies useful identities like:

$$
\begin{gathered}
\gamma_{1}<\gamma_{2} \Leftrightarrow \gamma_{1}<\gamma_{1}+\gamma_{2} \Leftrightarrow \gamma_{1}+\gamma_{2}<\gamma_{2}, \quad \gamma_{1}>\gamma_{3} \quad \text { if } \quad \gamma_{1}>\gamma_{2}, \quad \gamma_{2}>\gamma_{3} \\
\Theta\left(\gamma_{1}+\gamma_{2}, \gamma_{1}\right)=\Theta\left(\gamma_{2}, \gamma_{1}\right)=\Theta\left(\gamma_{2}, \gamma_{1}+\gamma_{2}\right)
\end{gathered}
$$

We shall also use the symbol $\Theta$ to denote the usual step function of a real variable

$$
\Theta(x)= \begin{cases}1 & \text { for } x \geq 0 \\ 00 & \text { for } x<0\end{cases}
$$

Which of the two definitions we are using in any given context can be understood by examining the argument of $\Theta$.

Since the sum in (1.3) runs over unordered set of $\alpha_{i}$ 's, we can choose a specific order of the $\left\{\alpha_{i}\right\}$ when we give the functional form of $g_{\text {ref }}$. We shall choose the convention in which the $\left\{\alpha_{i}\right\}$ 's are ordered as

$$
\alpha_{1}<\alpha_{2}<\alpha_{3} \cdots<\alpha_{n}
$$

In other words in the $2 \mathrm{D}$ plane $\alpha_{1}, \ldots \alpha_{n}$ form a clockwise order. We can also express (1.8) as

$$
\Theta\left(\alpha_{i}, \alpha_{j}\right)=\Theta(i-j), \quad \forall i, j
$$

Finally, we introduce the shorthand notation

$$
\alpha_{i j} \equiv\left\langle\alpha_{i}, \alpha_{j}\right\rangle
$$

In the rest of the paper, we shall not explicitly display the variable $y$ in the argument of $g_{\text {ref }}$ and other functions, but it should be understood that all the quantities depend on $y$.

\section{2. "Higgs branch" wall-crossing formula}

First, we shall describe the "Hhiggs branch" formula for $g_{\text {ref }}$, which will be denoted by $g_{\text {higgs }} \cdot g_{\text {higgs }}\left(\alpha_{1}, \ldots, \alpha_{n}\right)$ is given by the Poincare polynomial of a quiver with $n$ nodes, each carrying a $\mathrm{U}(1)$ factor, and with $\alpha_{i j}$ arrows directed from the $i$ th node to the $j$-th node for $i<j$. The latter in turn is given by the Reineke formula [45]. The algorithm for calculating $g_{\text {higgs }}$ 
following the original Reineke formula leads to many terms whose contributions cancel. We shall state the result using a slightly different but equivalent algorithm given in [30] (Section 3.3) where some of these cancellations are taken into account. Some applications of this formula can be found in $[48,49]$

Let $\sigma(i)$ for $1 \leq i \leq n$ denote a permutation of the numbers $1, \ldots, n$. $g_{\text {higgs }}$ is given as a sum over different permutations $\sigma$. It takes the form:

$$
\begin{aligned}
& g_{\text {higgs }}\left(\alpha_{1}, \ldots, \alpha_{n}\right)=(-1)^{-1+n}\left(y-y^{-1}\right)^{1-n} \\
& \times \sum_{\sigma} \mathrm{N}_{\text {higgs }}^{(n)}\left(\left\{\alpha_{i}\right\} ; \sigma\right)(-y)^{\sum_{l<k} \alpha_{\sigma(l) \sigma(k)}} \\
& =(-1)^{-1+n}(-y)^{-\sum_{i<j} \alpha_{i j}}\left(y-y^{-1}\right)^{1-n}
\end{aligned}
$$

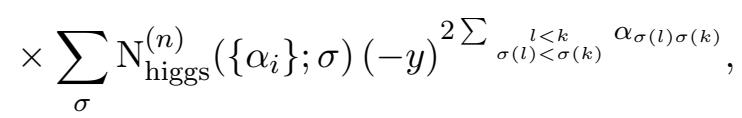

$$
\begin{aligned}
& \mathrm{N}_{\text {higgs }}^{(n)}\left(\left\{\alpha_{i}\right\} ; \sigma\right)=(-1)^{s(\sigma)-1} \prod_{\substack{k=2 \\
\sigma(k)<\sigma(k-1)}}^{n} \Theta\left(\alpha_{1}+\cdots \alpha_{n}, \sum_{i=k}^{n} \alpha_{\sigma(i)}\right) \\
& \times \prod_{\substack{k=2 \\
\sigma(k)>\sigma(k-1)}}^{n} \Theta\left(\sum_{i=k}^{n} \alpha_{\sigma(i)}, \alpha_{1}+\cdots \alpha_{n}\right) \\
& =(-1)^{s(\sigma)-1} \prod_{\substack{k=1 \\
\sigma(k+1)<\sigma(k)}}^{n-1} \Theta\left(\sum_{i=1}^{k} \alpha_{\sigma(i)}, \alpha_{1}+\cdots \alpha_{n}\right) \\
& \times \prod_{\substack{k=1 \\
\sigma(k+1)>\sigma(k)}}^{n-1} \Theta\left(\alpha_{1}+\cdots \alpha_{n}, \sum_{i=1}^{k} \alpha_{\sigma(i)}\right) \\
& s(\sigma)=1+\sum_{k=1}^{n-1} \Theta\left(\alpha_{\sigma(k)}, \alpha_{\sigma(k+1)}\right)=1+\sum_{k=1}^{n-1} \Theta(\sigma(k)-\sigma(k+1)) .
\end{aligned}
$$

The $\Theta$ in the second expression for $s(\sigma)$ is the ordinary step function. It has been shown in Appendix A that this is equivalent to the formula derived in Section 3.3 of [30] which in turn was shown in [30] to be equivalent to the Reineke formula [45]. The equality of the two expressions for $\mathrm{N}_{\text {higgs }}^{(n)}\left(\left\{\alpha_{i}\right\} ; \sigma\right)$ given in (2.1) follows from a simple shift $k \rightarrow k+1$ and the identities given 
in (1.6). Although for physical charges the $\alpha_{i j}$ 's are integers and hence $(2.1)$ is uniquely defined everywhere in the complex $y$-plane, we shall at the intermediate steps work with analytically continued $\alpha_{i j}$ 's away from integer values. In this case, we shall use (2.1) to define $g_{\text {higgs }}$ along the negative $y$-axis in the range $-1<y<0$ and then analytically continue the result to the rest of the complex plane. ${ }^{2}$

Since (2.1) will play a central role in our analysis, it will be useful to keep in mind a physical picture of this equation. What this equation tells us is that for a given permutation to contribute to $g_{\text {higgs }}$ it must satisfy the conditions:

$$
\begin{aligned}
& \sum_{i=1}^{k} \alpha_{\sigma(i)}<\alpha_{1}+\cdots+\alpha_{n} \quad \text { for } \sigma(k)<\sigma(k+1), \\
& \sum_{i=1}^{k} \alpha_{\sigma(i)}>\alpha_{1}+\cdots+\alpha_{n} \quad \text { for } \sigma(k)>\sigma(k+1) .
\end{aligned}
$$

Furthermore, when the above condition is satisfied, its contribution to $\mathrm{N}_{\text {higgs }}^{(n)}$ is 1 or -1 depending on whether the number of neighboring pairs for which $\sigma(i)>\sigma(i+1)$ is even or odd.

For $n=2$ the permutations are (12) and (21). Using (2.1) we get $s(12)=$ $1, s(21)=2, \mathrm{~N}_{\text {higgs }}^{(2)}(12)=(-1)^{s(12)-1} \Theta\left(\alpha_{1}+\alpha_{2}, \alpha_{1}\right)=\Theta\left(\alpha_{2}, \alpha_{1}\right)=1$ and $\mathrm{N}_{\text {higgs }}^{(2)}(21)=(-1)^{s(21)-1} \Theta\left(\alpha_{2}, \alpha_{1}+\alpha_{2}\right)=-\Theta\left(\alpha_{2}, \alpha_{1}\right)=-1$. Thus, we get

$$
g_{\text {higgs }}\left(\alpha_{1}, \alpha_{2}\right)=-(-y)^{-\alpha_{12}}\left(y-y^{-1}\right)^{-1}\left((-y)^{2 \alpha_{12}}-1\right)
$$

We shall end this section by summarizing some useful properties of $g_{\text {higgs }}$ :

(1) $g_{\text {higgs }}$ contains a sum of exponents of the form $(-y)^{\sum_{i<j} s_{i j} \alpha_{i j}}$ where $s_{i j}=$ 1 or -1 . Since there are $n(n-1) / 2$ pairs of $\alpha_{i j}$ 's, there are $2^{n(n-1) / 2}$

\footnotetext{
${ }^{2} \mathrm{~A}$ physical interpretation of the exponent $\sum_{l<k} \alpha_{\sigma(l) \sigma(k)}$ is as follows. Let us represent the $\alpha_{i}$ 's as vectors in the $2 \mathrm{D}$ plane such that $\alpha_{i j}$ is the area of the parallelogram with sides $\alpha_{i}$ and $\alpha_{j}$. Then $\sum_{l<k} \alpha_{\sigma(l) \sigma(k)}$ is the area of the oriented polygon with sides $\alpha_{\sigma(1)}, \alpha_{\sigma(2)}, \ldots, \alpha_{\sigma(n)}$ and $-\alpha_{\sigma(1)}-\cdots-\alpha_{\sigma(n)}$. In general, the polygon can be self-intersecting in which case the area has to be taken as the sum of the areas of each component polygon weighted with \pm 1 depending on the orientation of the boundary of that particular component. I wish to thank the referee for suggesting this interpretation.
} 
possible choices of the $\left\{s_{i j}\right\}$ 's. However, of these only those terms which have the form:

$$
(-y)^{\sum_{\substack{i<j \\ \sigma(i)<\sigma(j)}} \alpha_{\sigma(i) \sigma(j)}-\sum_{\substack{i>j \\ \sigma(i)<\sigma(j)}} \alpha_{\sigma(i) \sigma(j)}}=(-y)^{\sum_{i<j} \alpha_{\sigma(i) \sigma(j)}},
$$

for some permutation $\sigma$ appear in the sum. This already restricts the sum to $n$ ! terms corresponding to $n$ ! possible choices of $\sigma$. The constraints (2.2) further reduce the number of terms.

(2) Let $A_{a}\left(1 \leq a \leq 2^{n}-1\right)$ denote the collection of all non-empty subsets of $\{1,2, \ldots n\}$ and let

$$
\gamma^{(a)} \equiv \sum_{i \in A_{a}} \alpha_{i}
$$

Then $\mathrm{N}_{\text {higgs }}^{(n)}\left(\left\{\alpha_{i}\right\} ; \sigma\right)$ depends only on the relative orientation of the vectors $\gamma^{(a)}$ relative to $\left(\alpha_{1}+\cdots+\alpha_{n}\right)$, but not on the relative orientations of $\gamma^{(a)}$ and $\gamma^{(b)}$. This is apparent from the fact that the argument of the $\Theta$ 's appearing in (2.1) involve only the pairs $\left(\gamma^{(a)}, \alpha_{1}+\cdots+\alpha_{n}\right)$ but $\operatorname{not}\left(\gamma^{(a)}, \gamma^{(b)}\right)$.

(3) We can improve upon the above result if we focus on the term corresponding to a given permutation $\sigma$. The corresponding $\mathrm{N}_{\text {higgs }}^{(n)}\left(\left\{\alpha_{i}\right\} ; \sigma\right)$ depends only on the relative orientation of $\sum_{i=1}^{k} \alpha_{\sigma(i)}$ and $\alpha_{1}+\cdots+\alpha_{n}$ for $1 \leq k \leq n-1$, and of course the relative orientation of the pairs $\left(\alpha_{i}, \alpha_{j}\right)$. All the other $\gamma^{(a)}$ 's are irrelevant. Thus while computing $\mathrm{N}_{\text {higgs }}^{(n)}\left(\left\{\alpha_{i}\right\} ; \sigma\right)$ for a particular $\sigma$ we can freely deform the $\alpha_{i}$ 's as long as we do not change the relative orientation between $\sum_{i=1}^{k} \alpha_{\sigma(i)}$ and $\alpha_{1}+$ $\cdots+\alpha_{n}$ for any $k$, and also preserve the relative orientation between the $\alpha_{i}$ 's.

(4) If a permutation $\sigma$ appears in the sum in (2.1), then the permutation $\sigma^{\prime}$ where the order of all the elements is reversed, also appears in the sum. Proof: We have

$$
\sigma^{\prime}(i)=\sigma(n+1-i)
$$


Equation (2.1) now gives

$$
\begin{aligned}
& \mathrm{N}_{\text {higgs }}^{(n)}\left(\left\{\alpha_{i}\right\} ; \sigma^{\prime}\right)=(-1)^{s\left(\sigma^{\prime}\right)-1} \prod_{\substack{k=1 \\
\sigma^{\prime}(k+1)<\sigma^{\prime}(k)}}^{n-1} \Theta\left(\sum_{i=1}^{k} \alpha_{\sigma^{\prime}(i)}, \alpha_{1}+\cdots+\alpha_{n}\right) \\
& \times \prod_{\substack{k=1 \\
\sigma^{\prime}(k+1)>\sigma^{\prime}(k)}}^{n-1} \Theta\left(\alpha_{1}+\cdots+\alpha_{n}, \sum_{i=1}^{k} \alpha_{\sigma^{\prime}(i)}\right) \\
& =(-1)^{s\left(\sigma^{\prime}\right)-1} \prod_{\substack{k=1 \\
\sigma(n-k)<\sigma(n-k+1)}}^{n-1} \\
& \times \Theta\left(\sum_{i=1}^{k} \alpha_{\sigma(n-i+1)}, \alpha_{1}+\cdots+\alpha_{n}\right) \\
& \times \prod_{\substack{k=1 \\
\sigma(n-k)>\sigma(n-k+1)}}^{n-1} \Theta\left(\alpha_{1}+\cdots+\alpha_{n}, \sum_{i=1}^{k} \alpha_{\sigma(n-i+1)}\right), \\
& =(-1)^{s\left(\sigma^{\prime}\right)-1} \prod_{\substack{\ell=1 \\
\sigma(\ell)<\sigma(\ell+1)}}^{n-1} \Theta\left(\sum_{j=\ell+1}^{n} \alpha_{\sigma(j)}, \alpha_{1}+\cdots+\alpha_{n}\right) \\
& \times \prod_{\substack{\ell=1 \\
\sigma(\ell)>\sigma(\ell+1)}}^{n-1} \Theta\left(\alpha_{1}+\cdots+\alpha_{n}, \sum_{j=\ell+1}^{n} \alpha_{\sigma(j)}\right)
\end{aligned}
$$

with

$$
\begin{aligned}
s\left(\sigma^{\prime}\right) & =1+\sum_{k=1}^{n-1} \Theta\left(\sigma^{\prime}(k)-\sigma^{\prime}(k+1)\right)=1+\sum_{k=1}^{n-1} \Theta(\sigma(n-k+1)-\sigma(n-k)), \\
& =1+\sum_{j=1}^{n-1} \Theta(\sigma(j+1)-\sigma(j))=1+(n-1)-\sum_{j=1}^{n-1} \Theta(\sigma(j)-\sigma(j+1)) .
\end{aligned}
$$

Comparing (2.7), (2.8) with (2.1) we get

$$
\mathrm{N}_{\text {higgs }}^{(n)}\left(\left\{\alpha_{i}\right\} ; \sigma^{\prime}\right)=(-1)^{n-1} \mathrm{~N}_{\text {higgs }}^{(n)}\left(\left\{\alpha_{i}\right\} ; \sigma\right),
$$


showing that $\mathrm{N}_{\text {higgs }}^{(n)}\left(\left\{\alpha_{i}\right\} ; \sigma^{\prime}\right)$ is non-zero if $\mathrm{N}_{\text {higgs }}^{(n)}\left(\left\{\alpha_{i}\right\} ; \sigma\right)$ is non-zero. Since reversing the permutation reverses the sign of $\sum_{i<j} \alpha_{\sigma(i) \sigma(j)}$, the result given above shows that $g_{\text {higgs }}$ is invariant under $y \rightarrow y^{-1}$. This is of course expected from the fact that $g_{\text {higgs }}$ is the Poincare polynomial of the moduli space of abelian quivers.

Finally note that if we are interested in the ordinary (numerical) index instead of the refined index, the relevant $g$ is obtained by taking the $y \rightarrow 1$ limit of (2.1) [49]. This limit is apparently singular, but given that $g_{\text {higgs }}\left(\alpha_{1}, \ldots, \alpha_{n}\right)$ is the Poincare polynomial of abelian quivers and hence has a finite $y \rightarrow 1$ limit, the singularities must cancel after we sum over all permutations $\sigma$. Thus if we define $y=e^{\nu}$ and expand $\sum_{\sigma} \mathrm{N}_{\text {higgs }}^{(n)}\left(\left\{\alpha_{i}\right\} ; \sigma\right)$ $(-y)^{\sum_{l<k} \alpha_{\sigma(l) \sigma(k)}}$ in a power series in $\nu$, all powers of $\nu$ up to $\nu^{n-2}$ must cancel. As a result we can extract the $y \rightarrow 1$ limit of $g_{\text {higgs }}$ by picking the order $\nu^{n-1}$ term from the expansion of each $(-y)^{\sum_{l<k} \alpha_{\sigma(l) \sigma(k)}}$ term, and then taking the $\nu \rightarrow 0$ limit of the resulting expression. This gives,

$$
\begin{aligned}
& g_{\text {numerical }}\left(\alpha_{1}, \ldots, \alpha_{n}\right)=(-1)^{-1+n} 2^{1-n} \frac{1}{(n-1) !} \\
& \quad \times \sum_{\sigma} \mathrm{N}_{\text {higgs }}^{(n)}\left(\left\{\alpha_{i}\right\} ; \sigma\right)(-1)^{\sum_{l<k} \alpha_{\sigma(l) \sigma(k)}}\left(\sum_{l<k} \alpha_{\sigma(l) \sigma(k)}\right)^{n-1} \\
& \quad \rightarrow(-1)^{-1+n} 2^{1-n} \frac{1}{(n-1) !}(-1)^{\sum_{l<k} \alpha_{l k}} \\
& \quad \times \sum_{\sigma} \mathrm{N}_{\text {higgs }}^{(n)}\left(\left\{\alpha_{i}\right\} ; \sigma\right)\left(\sum_{l<k} \alpha_{\sigma(l) \sigma(k)}\right)^{n-1},
\end{aligned}
$$

where the second expression is valid in the limit when the $\alpha_{i}$ 's, instead of being general $2 \mathrm{D}$ vectors, approach lattice vectors so that $\alpha_{i j}$ 's approach integers. For generic charges (2.10) appears to be closely related to, but not quite the same as the JS wall-crossing formula [14-16]. (A short review of the JS formula and its implementation can be found in [30], Section 5.) In particular, in the JS formula the summand $\left(\sum_{l<k} \alpha_{\sigma(l) \sigma(k)}\right)^{n-1}$ is replaced by a slightly different term obtained by summing over trees. However, for non-generic charges i.e., when some $\alpha_{i}$ 's - and/or their linear combinations with positive integer coefficients - are equal or parallel to each other, the JS prescription involves sum over many more terms, while the MPS prescription simply requires us to take the limit of the formula for generic charges and supply the Boltzmann factor $1 /\left|\operatorname{Aut}\left(\left\{\alpha_{i}\right\}\right)\right|=1 / \prod_{k} m_{k}$ ! as described 
in (1.3). It will be interesting to find a direct combinatoric proof of the equivalence of (2.10) with the JS wall-crossing formula.

\section{KS wall-crossing formula}

We shall now describe a version of the KS wall-crossing formula given in [30]. To describe the KS wall-crossing formula, we introduce an algebra with elements of the form $e_{\gamma}$ with $\gamma \in \Lambda$, satisfying the commutation relations:

$$
\left[e_{\gamma}, e_{\gamma^{\prime}}\right]=\kappa\left(\gamma, \gamma^{\prime}\right) e_{\gamma+\gamma^{\prime}}, \quad \kappa\left(\gamma, \gamma^{\prime}\right)=\frac{(-y)^{\left\langle\gamma, \gamma^{\prime}\right\rangle}-(-y)^{-\left\langle\gamma, \gamma^{\prime}\right\rangle}}{y-y^{-1}}
$$

Let $\alpha_{1}, \ldots, \alpha_{n}$ be a set of vectors arranged so that $\alpha_{1}<\alpha_{2}<\cdots<\alpha_{n}$, i.e., in the $2 \mathrm{D}$ representation $\alpha_{1}, \ldots, \alpha_{n}$ are arranged in a clockwise fashion. As before, we denote by $\left\{A_{a}\right\}$ the collection of all possible non-empty subsets of the integers $1, \ldots, n$, and define $\gamma^{(a)}=\sum_{i \in A^{(a)}} \alpha_{i}$. We shall order the $A_{a}$ 's so that $\gamma^{(a)}$ 's form a clockwise order as $a$ increases: $\gamma^{(a)}<\gamma^{(b)}$ for $a<b$. Now we begin with the product $e_{\alpha_{n}} \cdots e_{\alpha_{1}}$ and then try to reverse the order using (3.1), bringing this into a linear combination of terms of the form $e_{\gamma^{\left(a_{1}\right)}} e_{\gamma^{\left(a_{2}\right)}} \cdots e_{\gamma^{\left(a_{k}\right)}}$ with $a_{1}<a_{2}<\cdots<a_{k}, \gamma^{\left(a_{1}\right)}+\cdots+\gamma^{\left(a_{k}\right)}=$ $\alpha_{1}+\cdots+\alpha_{n}$ :

$$
\begin{aligned}
e_{\alpha_{n}} \cdots e_{\alpha_{1}}= & \sum_{k=1}^{n} \sum_{\substack{\left\{a_{1}, \ldots a_{k}\right\} \\
\gamma^{\left(a_{1}\right)}+\cdots \gamma^{\left(a_{k}\right)}=\alpha_{1}+\cdots+\alpha_{n} ; a_{1}<a_{2} \cdots<a_{k}}} \\
& \times h\left(\alpha_{1}, \ldots, \alpha_{n} ; \gamma^{\left(a_{1}\right)}, \ldots, \gamma^{\left(a_{k}\right)}\right) e_{\gamma^{\left(a_{1}\right)}} \cdots e_{\gamma^{\left(a_{k}\right)}},
\end{aligned}
$$

for some functions $h$. The $g_{\text {ref }}$ for KS wall-crossing formula, denoted by $g_{\mathrm{KS}}$, is given by the coefficient of $e_{\alpha_{1}+\cdots+\alpha_{n}}$ in this expression:

$$
g_{\mathrm{KS}}\left(\alpha_{1}, \ldots, \alpha_{n}\right)=h\left(\alpha_{1}, \ldots, \alpha_{n} ; \alpha_{1}+\cdots+\alpha_{n}\right)
$$

For example for $n=2$, we write

$$
e_{\alpha_{2}} e_{\alpha_{1}}=e_{\alpha_{1}} e_{\alpha_{2}}+\kappa\left(\alpha_{2}, \alpha_{1}\right) e_{\alpha_{1}+\alpha_{2}}
$$

Thus, we have

$$
g_{\mathrm{KS}}\left(\alpha_{1}, \alpha_{2}\right)=\kappa\left(\alpha_{2}, \alpha_{1}\right) .
$$


This agrees with the corresponding formula (2.3) for $g_{\text {higgs }}\left(\alpha_{1}, \alpha_{2}\right)$. The equivalence of $g_{\mathrm{KS}}\left(\alpha_{1}, \ldots, \alpha_{n}\right)$ and $g_{\text {higgs }}\left(\alpha_{1}, \ldots, \alpha_{n}\right)$ has been tested explicitly up to $n \leq 5[30]$.

We shall now examine if $g_{\mathrm{KS}}$ also satisfies the four properties of $g_{\mathrm{higgs}}$ listed at the end of Section 2.

(1) We shall first show that like in the expression for $g_{\text {higgs }}$ given in (2.1), each term in $g_{\mathrm{KS}}$ can also be associated with a permutation, i.e., $g_{\mathrm{KS}}$ can be expressed as

$$
\begin{aligned}
g_{\mathrm{KS}}\left(\alpha_{1}, \ldots, \alpha_{n}\right)= & (-1)^{-1+n}(-y)^{-\sum_{i<j} \alpha_{i j}}\left(y-y^{-1}\right)^{1-n} \\
& \times \sum_{\sigma} \mathrm{N}_{\mathrm{KS}}^{(n)}\left(\left\{\alpha_{i}\right\} ; \sigma\right)(-y)^{2 \sum_{\sigma(l)<\sigma(k)}^{l<k} \alpha_{\sigma(l) \sigma(k)}} \\
= & (-1)^{-1+n}\left(y-y^{-1}\right)^{1-n} \sum_{\sigma} \mathrm{N}_{\mathrm{KS}}^{(n)}\left(\left\{\alpha_{i}\right\} ; \sigma\right)(-y)^{\sum_{l<k} \alpha_{\sigma(l) \sigma(k)}}
\end{aligned}
$$

for some integers $\mathrm{N}_{\mathrm{KS}}^{(n)}\left(\left\{\alpha_{i}\right\} ; \sigma\right)$. Suppose we begin with a pair of generators $e_{\alpha_{i}}, e_{\alpha_{j}}$ and pick up their commutator. Then the coefficient of this term, besides the $\left(y-y^{-1}\right)^{-1}$ term, is proportional to $(-y)^{\alpha_{i j}}-$ $(-y)^{-\alpha_{i j}}$. The first term has the interpretation of a permutation in which $i$ is to the left of $j$ and the second term has the interpretation of being associated with a permutation in which $j$ appears to the left of $i$. If we now pick the commutator of $e_{\alpha_{i}+\alpha_{j}}$ with a third generator $e_{\alpha_{k}}$, then we get a factor proportional to $(-y)^{\alpha_{i k}+\alpha_{j k}}-(-y)^{-\alpha_{i k}-\alpha_{j k}}$. The first term has the interpretation of a permutation in which the $i$ and $j$ are to the left of $k$ and the second term has the interpretation of a permutation in which $k$ is to the left of $i$ and $j$. Thus, this can be combined with the earlier ordering $(i j)$ or $(j i)$ without any conflict. ${ }^{3}$ This argument can be extended to more general situations. Individual steps in arriving at (3.2) consists of manipulating a product $e_{\gamma^{(a)}} e_{\gamma^{(b)}}$ by reversing their order where $\gamma^{(a)}$ and $\gamma^{(b)}$ are defined as in (2.5) with non-overlapping sets $A_{a}$ and $A_{b}$. Now while reversing the order in the product $e_{\gamma^{(a)}} e_{\gamma^{(b)}}$ the commutator gives $e_{\gamma^{(a)}+\gamma^{(b)}}$ multiplied by a factor proportional to $(-y)^{\left\langle\gamma^{(a)}, \gamma^{(b)}\right\rangle}-(-y)^{\left\langle\gamma^{(b)}, \gamma^{(a)}\right\rangle}$. The first term can be regarded as coming from a configuration where all the elements of $A_{a}$

\footnotetext{
${ }^{3}$ In contrast if we had found a term like $\alpha_{i k}-\alpha_{j k}-\alpha_{i j}$ in the exponent it would have the interpretation that $k$ is to the right of $i$ and left of $j$, and $j$ is to the left of $i$. Clearly there is no arrangement of $i, j, k$ satisfying these requirements.
} 
are to the left of all the elements of $A_{b}$ and the second term can be regarded as coming from a configuration where all the elements of $A_{a}$ are to the right of all the elements of $A_{b}$. Suppose further that earlier, while arriving at $e_{\gamma^{(a)}}$ by combining $e_{\alpha_{i}}$ for $i \in A_{a}$ we have gotten a sum of terms each of which can be associated with the permutation of the elements inside $A_{a}$ and a similar relation holds for $e_{\gamma^{(b)}}$. When we multiply these by the $(-y)^{\left\langle\gamma^{(a)}, \gamma^{(b)}\right\rangle}-(-y)^{\left\langle\gamma^{(b)}, \gamma^{(a)}\right\rangle}$ factor, individual terms in the product will correspond to specific permutation of the elements inside $A_{a}$ and specific permutation of the elements inside $A_{b}$, and on top of that all the elements of $A_{a}$ could be to the left of all the elements of $A_{b}$ or all the elements of $A_{b}$ could be to the left of all the elements of $A_{a}$. Thus each term multiplying $e_{\gamma^{(a)}+\gamma^{(b)}}$ can be regarded as coming from some permutation of the elements of $A_{a} \cup A_{b}$. This now shows by induction that at every stage of the manipulation that leads to the KS formula for wall crossing, we produce a set of terms each of which can be associated with a permutation of the $\alpha_{i}$ 's involved. As a result the final expression for $g_{\mathrm{KS}}$ must also contain only those powers of $y$ which have the interpretation of being associated with a permutation as in (3.6). We shall see this more explicitly in (5.4), (5.5) and (5.7).

(2) Like $g_{\text {higgs }}, g_{\mathrm{KS}}$ is also a piecewise analytic function of the $\alpha_{i}$ 's. The form of the function depends on the relative orientation between $\gamma^{(a)}$ and $\alpha_{1}+\cdots+\alpha_{n}$ and is independent of the relative orientation between the $\gamma^{(a)}$ 's. This was proved in [30], but for completeness we shall repeat the proof. Let us suppose that by manipulating the product $e_{\alpha_{n}} \cdots e_{\alpha_{1}}$ we have brought it into the form (3.2). Since $\kappa\left(\gamma, \gamma^{\prime}\right)$ is an analytic function of $\gamma, \gamma^{\prime}$, it follows from (3.1) that the coefficient of $e_{\alpha_{1}+\cdots+\alpha_{n}}$ in this expression, which is given by sum of products of $\kappa\left(\gamma, \gamma^{\prime}\right)$ with $\gamma=\sum_{i \in A} \alpha_{i}, \gamma^{\prime}=\sum_{i \in B} \alpha_{i}$ for some subsets $A$ and $B$ of $\{1,2, \ldots, n\}$, is an analytic function of the $\alpha_{i}$ 's inside a chamber in which the relative order of the $\gamma^{(a)}$ 's is fixed. Now suppose that we deform some of the $\alpha_{i}$ 's to make a pair of $\gamma^{(a)}$ 's switch their relative orientation but none of the $\gamma^{(a)}$ 's cross the ray corresponding to $\alpha_{1}+\cdots+\alpha_{n}$. In particular let us suppose that the relevant pairs are $\gamma^{(b)}$ and $\gamma^{(c)}$, and that the deformation takes us from $\gamma^{(b)}<\gamma^{(c)}$ to $\gamma^{(b)}>\gamma^{(c)}$. In this case, $\gamma^{(b)}$ and $\gamma^{(c)}$ are either both on the left or both on the right of $\alpha_{1}+\cdots+\alpha_{n}$. Let us for definiteness assume that they are both to the left. Now to bring the products of $e_{\gamma^{(a)}}$ 's to the standard order we need to express the product $e_{\gamma^{(b)}} e_{\gamma^{(c)}}$ inside any term as $e_{\gamma^{(c)}} e_{\gamma^{(b)}}+\kappa\left(\gamma^{(b)}, \gamma^{(c)}\right) e_{\gamma^{(b)}+\gamma^{(c)}}$. However 
since $\gamma^{(b)}$ and $\gamma^{(c)}$ are both on the left of $\alpha_{1}+\cdots+\alpha_{n}$, the extra term proportional to $e_{\gamma^{(b)}+\gamma^{(c)}}$ can never give a factor of $e_{\alpha_{1}+\cdots \alpha_{n}}$. Thus under such deformations $g_{\mathrm{KS}}\left(\alpha_{1}, \ldots, \alpha_{n}\right)$, which is the coefficient of $e_{\alpha_{1}+\cdots+\alpha_{n}}$ at the end of this manipulation, remains unchanged. This shows that $g_{\mathrm{KS}}\left(\alpha_{1}, \ldots, \alpha_{n}\right)$ is a piecewise analytic function of the $\alpha_{i}$ 's, with the form of the function determined by the relative orientation between the $\gamma^{(a)}$ 's and $\alpha_{1}+\cdots+\alpha_{n}$.

(3) As in the case of $g_{\text {higgs }}$, one can improve the result if we focus on a term corresponding to a given permutation $\sigma$. We shall show that in this case the coefficient depends only on the relative orientation of $\sum_{i=1}^{k} \alpha_{\sigma(i)}$ and $\alpha_{1}+\cdots+\alpha_{n}$ for different values of $k$, and not on the relative orientation between $\gamma^{(a)}$ and $\alpha_{1}+\cdots+\alpha_{n}$ for other $\gamma^{(a)}$ 's. To see this let us again suppose that by manipulating the product $e_{\alpha_{n}} \cdots e_{\alpha_{1}}$ we have brought it into the form (3.2). Now suppose that we deform the $\alpha_{i}$ 's so that a specific $\gamma^{(a)}$ crosses $\alpha_{1}+\cdots+\alpha_{n}$ from left to right. At the same time the vector $\gamma^{(b)} \equiv \alpha_{1}+\cdots+\alpha_{n}-\gamma^{(a)}$ crosses $\alpha_{1}+\cdots+\alpha_{n}$ from right to left. Before deformation the generators $e_{\gamma^{(a)}}$ and $e_{\gamma^{(b)}}$ would have been arranged in the order $e_{\gamma^{(a)}} e_{\gamma^{(b)}}$, but after the deformation we need to reverse their order picking up a term proportional to $\left\{(-y)^{\left\langle\gamma^{(a)}, \gamma^{(b)}\right\rangle}-(-y)^{\left\langle\gamma^{(b)}, \gamma^{(a)}\right\rangle}\right\} e_{\alpha_{1}+\cdots+\alpha_{n}}$. The first term has the interpretation of all the elements in the set $A_{a}$ being to the left of all the elements in the set $A_{b}$ (which is the complement of the set $A_{a}$ ) and the second term has the interpretation of all the elements in the set $A_{b}$ being to the left of all the elements in the set $A_{a}$. Thus, such a term can change the coefficient of a term associated with the permutation $\sigma$ only if in $\sigma$ all the elements of $A_{a}$ are to the left (or right) of all the elements of the compliment of $A_{a}$. In other words $A_{a}$ must contain a set of $k$ elements to the left (or a set of $(n-k)$ elements to the right) for some integer $k$. This shows that the coefficient of a term associated with the permutation $\sigma$ in $g_{\mathrm{KS}}$ can only depend on the relative orientation between $\alpha_{\sigma(1)}+\cdots+\alpha_{\sigma(k)}$ and $\alpha_{1}+\cdots+\alpha_{n}$ for different integers $k$ but not on the relative orientation between $\gamma^{(a)}$ and $\alpha_{1}+\cdots+\alpha_{n}$ for other sets $A_{a}$.

(4) Finally, we turn to the fourth property of $g_{\text {higgs }}$ that states that if a permutation $\sigma$ appears in $g_{\text {higgs }}$ then its reverse permutation will also appear. As discussed at the end of Section 2, this is equivalent to proving the symmetry of $g_{\text {higgs }}$ under $y \rightarrow y^{-1}$. This property is automatic in $g_{\mathrm{KS}}$ since the $y$ dependence arises from the $\kappa\left(\gamma_{1}, \gamma_{2}\right)$ factors which are manifestly invariant under $y \rightarrow y^{-1}$. 
In the next two sections we shall prove the equality of $g_{\mathrm{KS}}\left(\alpha_{1}, \ldots, \alpha_{n}\right)$ and $g_{\text {higgs }}\left(\alpha_{1}, \ldots, \alpha_{n}\right)$ for generic charge vectors $\left\{\alpha_{i}\right\}$ for which all vectors of the form $\sum_{i \in A} \alpha_{i}$ for different subsets $A$ of $\{1, \ldots, n\}$ are strictly ordered. The special cases where some of these vectors are parallel or identical to each other are then obtained as limits of this generic case. One question that arises naturally is whether the limit is well defined, i.e., whether it could depend on which side we approach the limit from. This is somewhat obscure for the expression (2.1) for $g_{\text {higgs }}$ since it involves the step functions which jump discontinuously as the relative ordering between the vectors in the argument switch. However for $g_{\mathrm{KS}}$ defined through (3.2), (3.3) it is clear that the limit is well defined, since the effect of switching the order between two vectors $\gamma$ and $\gamma^{\prime}$ vanishes as $\gamma$ and $\gamma^{\prime}$ become parallel to each other: $e_{\gamma} e_{\gamma^{\prime}} \rightarrow e_{\gamma^{\prime}} e_{\gamma}$ when $\gamma$ and $\gamma^{\prime}$ are parallel. Thus for example if we approach a configuration where two vectors $\alpha_{i}$ and $\alpha_{j}$ become parallel to each other, then the left-hand side of (3.2) is independent of whether they approach this configuration from the $\alpha_{i}>\alpha_{j}$ side or $\alpha_{i}<\alpha_{j}$ side. Similarly if a pair of $\gamma^{(a)}$ 's become parallel to each other, then $h\left(\alpha_{1}, \ldots, \alpha_{n} ; \alpha_{1}+\cdots+\alpha_{n}\right)$ appearing on the right-hand side of (3.2) is independent of how this limit is approached. Equation (3.3) then shows that for $g_{\mathrm{KS}}$ the limit to degenerate configuration of vectors is well defined. The equality of $g_{\mathrm{KS}}$ and $g_{\text {higgs }}$ for generic vectors, which will be proved in the next two sections, then implies that even for $g_{\text {higgs }}$ the limit to degenerate configurations of vectors is well defined, i.e., it does not depend on which side we take the limit from.

We must reemphasize however that for degenerate configurations of vectors $g_{\text {higgs }}$ must be defined as a limit of (2.1) for non-degenerate configurations. For example, if we were to write a computer program for computing $g_{\text {higgs }}$, the algorithm must involve adding to the $\alpha_{i}$ 's some randomly generated $2 \mathrm{D}$ vectors of small magnitude - which makes the configuration non-degenerate - while computing $N_{\text {higgs }}^{(n)}\left(\left\{\alpha_{i}\right\} ; \sigma\right)$, but while computing the exponent $\sum_{l<k} \alpha_{\sigma(l) \sigma(k)}$ of $(-y)$ we can continue to use the original vectors. For a fixed permutation $\sigma$ the quantities $N_{\text {higgs }}^{(n)}\left(\left\{\alpha_{i}\right\} ; \sigma\right)$ may depend on the choice of the random vectors which we add to the $\alpha_{i}$ 's, but the argument of the previous paragraph shows that the final result for $g_{\text {higgs }}\left(\alpha_{1}, \ldots, \alpha_{n}\right)$ will be independent of this choice.

\section{Recursion relations for the KS wall-crossing formula}

We shall now derive a set of recursion relations for $g_{\mathrm{KS}}\left(\alpha_{1}, \ldots, \alpha_{n}\right)$. Since $\gamma^{(a)}=\sum_{i \in A_{a}} \alpha_{i}$, it is clear that the $e_{\gamma^{(a)}}$ factor in (3.2) arises as a result 
of manipulating the product of $e_{\alpha_{i}}$ 's for $i \in A_{a}$, to bring it from the anticlockwise ordering to the clockwise ordering. Furthermore the result of this manipulation is not affected by the $\alpha_{i}$ 's outside the set $A_{a}$, and hence gives a factor of $g_{\mathrm{KS}}\left(\left\{\alpha_{i}, i \in A_{a}\right\}\right)$. Thus we get

$$
h\left(\alpha_{1}, \ldots \alpha_{n} ; \gamma^{\left(a_{1}\right)}, \ldots \gamma^{\left(a_{k}\right)}\right)=\prod_{\ell=1}^{k} g_{\mathrm{KS}}\left(\left\{\alpha_{i}, i \in A_{a_{\ell}}\right\}\right)
$$

Using this, we may rewrite (3.2) as

$$
\begin{aligned}
e_{\alpha_{n}} \ldots e_{\alpha_{1}}= & \sum_{k=1}^{n} \sum_{\substack{\left.\gamma^{\left(a_{1}\right)}+\cdots \gamma^{\left(a_{k}\right)}=\alpha_{1}, \ldots a_{k}\right\} \\
\alpha_{n} ; a_{1}<a_{2} \cdots<a_{k}}} \\
& \times\left(\prod_{\ell=1}^{k} g_{\mathrm{KS}}\left(\left\{\alpha_{i}, i \in A_{a_{\ell}}\right\}\right)\right) e_{\gamma^{\left(a_{1}\right)} \ldots e_{\gamma^{\left(a_{k}\right)}} .}
\end{aligned}
$$

We shall now use (4.2) to derive a recursive procedure for determining $g_{\mathrm{KS}}$. Suppose we know the result for $g_{\mathrm{KS}}\left(\alpha_{1}, \ldots, \alpha_{n}\right)$. Then to find $g_{\mathrm{KS}}\left(\alpha_{1}, \ldots, \alpha_{n}, \alpha_{n+1}\right)$ with $\alpha_{1}<\alpha_{2}<\cdots<\alpha_{n}<\alpha_{n+1}$, we multiply Equation (4.2) from the left by $e_{\alpha_{n+1}}$, and then try to rearrange the right-hand side by moving $e_{\alpha_{n+1}}$ to the extreme right, so that in each product the $e_{\gamma}$ 's have their $\gamma$ 's in clockwise order as we move from left to right. For example, in the first step we write

$$
\begin{aligned}
e_{\alpha_{n+1}} e_{\gamma^{\left(a_{1}\right)}} e_{\gamma^{\left(a_{2}\right)}} \cdots e_{\gamma^{\left(a_{k}\right)}}= & e_{\gamma^{\left(a_{1}\right)}} e_{\alpha_{n+1}} e_{\gamma^{\left(a_{2}\right)}} \cdots e_{\gamma^{\left(a_{k}\right)}} \\
& +\kappa\left(\alpha_{n+1}, \gamma^{\left(a_{1}\right)}\right) e_{\gamma^{\left(a_{1}\right)}+\alpha_{n+1}} e_{\gamma^{\left(a_{2}\right)}} \cdots e_{\gamma^{\left(a_{k}\right)}}
\end{aligned}
$$

In the next step, we manipulate the first term as

$$
\begin{aligned}
& e_{\gamma^{\left(a_{1}\right)}} e_{\alpha_{n+1}} e_{\gamma^{\left(a_{2}\right)}} e_{\gamma^{\left(a_{3}\right)}} \cdots e_{\gamma^{\left(a_{k}\right)}} \\
& =e_{\gamma^{\left(a_{1}\right)}} e_{\gamma^{\left(a_{2}\right)}} e_{\alpha_{n+1}} e_{\gamma^{\left(a_{3}\right)}} \cdots e_{\gamma^{\left(a_{k}\right)}} \\
& \quad+\kappa\left(\alpha_{n+1}, \gamma^{\left(a_{2}\right)}\right) e_{\gamma^{\left(a_{1}\right)}} e_{\gamma^{\left(a_{2}\right)}+\alpha_{n+1}} e_{\gamma^{\left(a_{3}\right)}} \cdots e_{\gamma^{\left(a_{k}\right)}} .
\end{aligned}
$$

For the second term of (4.3) we have to consider two possibilities. If $\gamma^{\left(a_{1}\right)}+$ $\alpha_{n+1}<\gamma^{\left(a_{2}\right)}$ we already have all the terms in the product in the correct order and we can stop manipulating this term. On the other hand, 
if $\gamma^{\left(a_{1}\right)}+\alpha_{n+1}>\gamma^{\left(a_{2}\right)}$ we write

$$
\begin{aligned}
& \kappa\left(\alpha_{n+1}, \gamma^{\left(a_{1}\right)}\right) e_{\gamma^{\left(a_{1}\right)}+\alpha_{n+1}} e_{\gamma^{\left(a_{2}\right)}} e_{\gamma^{\left(a_{3}\right)}} \cdots e_{\gamma^{\left(a_{k}\right)}} \\
& \quad=\kappa\left(\alpha_{n+1}, \gamma^{\left(a_{1}\right)}\right) e_{\gamma^{\left(a_{2}\right)}} e_{\gamma^{\left(a_{1}\right)}+\alpha_{n+1}} e_{\gamma^{\left(a_{3}\right)}} \cdots e_{\gamma^{\left(a_{k}\right)}} \\
& \quad+\kappa\left(\alpha_{n+1}, \gamma^{\left(a_{1}\right)}\right) \kappa\left(\alpha_{n+1}+\gamma^{\left(a_{1}\right)}, \gamma^{\left(a_{2}\right)}\right) e_{\gamma^{\left(a_{1}\right)}+\gamma^{\left(a_{2}\right)}+\alpha_{n+1}} e_{\gamma^{\left(a_{3}\right)}} \cdots e_{\gamma^{\left(a_{k}\right)}}
\end{aligned}
$$

In the next step, we shall need to manipulate the product of $e_{\gamma^{\left(a_{3}\right)}}$ with the terms to its left and so on.

To extract $g_{\mathrm{KS}}\left(\alpha_{1}, \ldots, \alpha_{n+1}\right)$ from this we have to determine the coefficients of $e_{\alpha_{1}+\cdots+\alpha_{n+1}}$. Now by examining (4.3) we can see that the first term on the right-hand side can never contribute to this sum. This is because we have $\gamma^{\left(a_{1}\right)}<\alpha_{n+1}, \gamma^{\left(a_{2}\right)}, \ldots, \gamma^{\left(a_{k}\right)}$. Thus, whatever manipulation we do to bring $e_{\gamma^{\left(a_{1}\right)}} e_{\alpha_{n+1}} e_{\gamma^{\left(a_{2}\right)}} \cdots e_{\gamma^{\left(a_{n}\right)}}$ in the clockwise order, the $e_{\gamma^{\left(a_{1}\right)}}$ will never be involved in the manipulation and continue to sit at the left. Thus every term that we get from this will have an $e_{\gamma^{\left(a_{1}\right)}}$ factor at the extreme left and we shall never get $e_{\alpha_{1}+\cdots+\alpha_{n+1}}$. By the same logic, the first term on the right-hand side of (4.5) will never produce $e_{\alpha_{1}+\cdots+\alpha_{n+1}}$. By repeated use of this logic we see that the only term in $e_{\alpha_{n+1}} e_{\gamma^{\left(a_{1}\right)}} e_{\gamma^{\left(a_{2}\right)}} \cdots e_{\gamma^{\left(a_{k}\right)}}$ proportional to $e_{\alpha_{1}+\cdots+\alpha_{n+1}}$ is given by

$$
\begin{aligned}
& \kappa\left(\alpha_{n+1}, \gamma^{\left(a_{1}\right)}\right) \kappa\left(\alpha_{n+1}+\gamma^{\left(a_{1}\right)}, \gamma^{\left(a_{2}\right)}\right) \cdots \kappa\left(\alpha_{n+1}\right. \\
& \left.\quad+\gamma^{\left(a_{1}\right)}+\cdots \gamma^{\left(a_{k-1}\right)}, \gamma^{\left(a_{k}\right)}\right) e_{\alpha_{n+1}+\gamma^{\left(a_{1}\right)}+\cdots \gamma^{\left(a_{k}\right)}}
\end{aligned}
$$

and furthermore this term exists only under the condition

$$
\begin{gathered}
\alpha_{n+1}+\gamma^{\left(a_{1}\right)}>\gamma^{\left(a_{2}\right)}, \quad \alpha_{n+1}+\gamma^{\left(a_{1}\right)}+\gamma^{\left(a_{2}\right)}>\gamma^{\left(a_{3}\right)}, \quad \ldots \\
\alpha_{n+1}+\gamma^{\left(a_{1}\right)}+\cdots+\gamma^{\left(a_{k-1}\right)}>\gamma^{\left(a_{k}\right)}
\end{gathered}
$$

Using (4.2) we now get

$$
\begin{aligned}
g_{\mathrm{KS}}\left(\alpha_{1}, \ldots, \alpha_{n+1}\right)= & \sum_{k=1}^{n} \sum_{\substack{\left.\gamma^{\left(a_{1}\right)}+\cdots+\gamma\left(a_{k}\right)=\alpha_{1}+\cdots+\alpha_{n} ; a_{1}<a_{2} \cdots<a_{k} \\
a_{1}, \ldots, a_{k}\right\}}} \\
& \times\left(\prod_{\ell=1}^{k} g_{\mathrm{KS}}\left(\left\{\alpha_{i}, i \in A_{a_{\ell}}\right\}\right)\right) \Theta\left(\alpha_{n+1}+\gamma^{\left(a_{1}\right)}, \gamma^{\left(a_{2}\right)}\right)
\end{aligned}
$$




$$
\begin{aligned}
& \times \Theta\left(\alpha_{n+1}+\gamma^{\left(a_{1}\right)}+\gamma^{\left(a_{2}\right)}, \gamma^{\left(a_{3}\right)}\right) \cdots \\
& \times \Theta\left(\alpha_{n+1}+\gamma^{\left(a_{1}\right)}+\cdots+\gamma^{\left(a_{k-1)},\right.}, \gamma^{\left(a_{k}\right)}\right) \\
& \times \kappa\left(\alpha_{n+1}, \gamma^{\left(a_{1}\right)}\right) \kappa\left(\alpha_{n+1}+\gamma^{\left(a_{1}\right)}, \gamma^{\left(a_{2}\right)}\right) \cdots \\
& \times \kappa\left(\alpha_{n+1}+\gamma^{\left(a_{1}\right)}+\cdots+\gamma^{\left(a_{k-1)}\right.}, \gamma^{\left(a_{k}\right)}\right),
\end{aligned}
$$

where $\Theta\left(\gamma_{1}, \gamma_{2}\right)$ has been defined in (1.5).

\section{Equivalence of KS and "Hhiggs branch" wall-crossing formulæ}

We shall now prove the equivalence of $g_{\mathrm{KS}}$ and $g_{\text {higgs }}$ using the method of induction, i.e., we shall assume that $g_{\mathrm{KS}}\left(\alpha_{1}, \ldots, \alpha_{m}\right)=g_{\text {higgs }}\left(\alpha_{1}, \ldots, \alpha_{m}\right)$ for $m \leq n$ and then prove the result for $m=n+1$. The equality of $g_{\mathrm{KS}}\left(\alpha_{1}, \alpha_{2}\right)$ and $g_{\text {higgs }}\left(\alpha_{1}, \alpha_{2}\right)$ will then imply the equivalence of $g_{\mathrm{KS}}\left(\alpha_{1}, \ldots, \alpha_{n}\right)$ and $g_{\text {higgs }}\left(\alpha_{1}, \ldots, \alpha_{n}\right)$ for all $n$.

\section{1. $g_{\mathrm{KS}}$ as a sum over permutations}

Assuming the equality of $g_{\mathrm{KS}}\left(\alpha_{1}, \ldots, \alpha_{m}\right)=g_{\text {higgs }}\left(\alpha_{1}, \ldots, \alpha_{m}\right)$ for $m \leq n$ we can replace $g_{\mathrm{KS}}$ by $g_{\text {higgs }}$ on the right-hand side of (4.8) and obtain

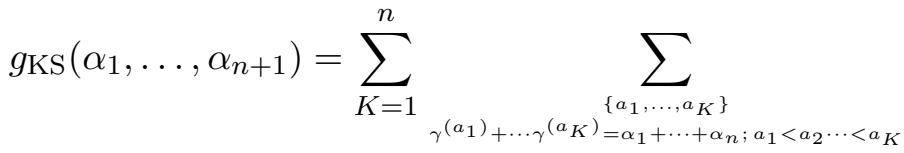

$$
\begin{aligned}
& \times\left(\prod_{\ell=1}^{K} g_{\text {higgs }}\left(\left\{\alpha_{i}, i \in A_{a_{\ell}}\right\}\right)\right) \Theta\left(\alpha_{n+1}+\gamma^{\left(a_{1}\right)}, \gamma^{\left(a_{2}\right)}\right) \\
& \times \Theta\left(\alpha_{n+1}+\gamma^{\left(a_{1}\right)}+\gamma^{\left(a_{2}\right)}, \gamma^{\left(a_{3}\right)}\right) \cdots \\
& \times \Theta\left(\alpha_{n+1}+\gamma^{\left(a_{1}\right)}+\cdots \gamma^{\left(a_{K-1)}\right.}, \gamma^{\left(a_{K}\right)}\right) \\
& \times \kappa\left(\alpha_{n+1}, \gamma^{\left(a_{1}\right)}\right) \kappa\left(\alpha_{n+1}+\gamma^{\left(a_{1}\right)}, \gamma^{\left(a_{2}\right)}\right) \cdots \\
& \times \kappa\left(\alpha_{n+1}+\gamma^{\left(a_{1}\right)}+\cdots+\gamma^{\left(a_{K-1)}\right.}, \gamma^{\left(a_{K}\right)}\right) \text {. }
\end{aligned}
$$

Note that we have replaced the summation variable $k$ by $K$ since soon we shall use the variable $k$ for other purposes. Let $n_{a}$ be the total number of elements in the set $A_{a}$ and let $I_{1}^{(a)}, \ldots, I_{n_{a}}^{(a)}$ be the elements of $A_{a}$, ordered so 
that $I_{1}^{(a)}<I_{2}^{(a)}<\cdots I_{n_{a}}^{(a)}$. After substituting the expression for $g_{\text {higgs }}$ given in $(2.1)$ we obtain

$$
\begin{aligned}
& g_{\mathrm{KS}}\left(\alpha_{1}, \ldots, \alpha_{n+1}\right)
\end{aligned}
$$

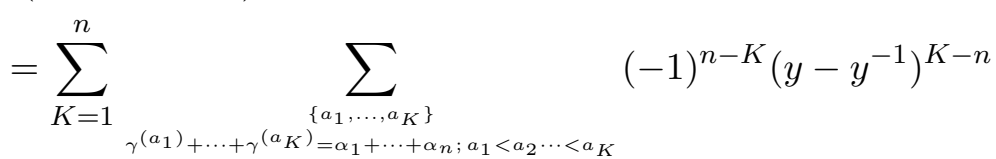

$$
\begin{aligned}
& \times \sum_{\substack{\bar{\sigma}\left(A_{a_{k}}\right)=A_{a_{k}} \forall k\\
}}\left[\left\{\prod_{\ell=1}^{K} \prod_{\substack{k=1 \\
\bar{\sigma}\left(I_{k+1}^{\left(a_{\ell}\right)}\right)<\bar{\sigma}\left(I_{k}^{\left(a_{\ell}\right)}\right)}}^{n_{a_{\ell}}-1} \Theta\left(\sum_{j=1}^{k} \alpha_{\bar{\sigma}\left(I_{j}^{\left(a_{\ell}\right)}\right)}, \sum_{j=1}^{n_{a_{\ell}}} \alpha_{\bar{\sigma}\left(I_{j}^{\left(a_{\ell}\right)}\right)}\right)\right\}\right.
\end{aligned}
$$

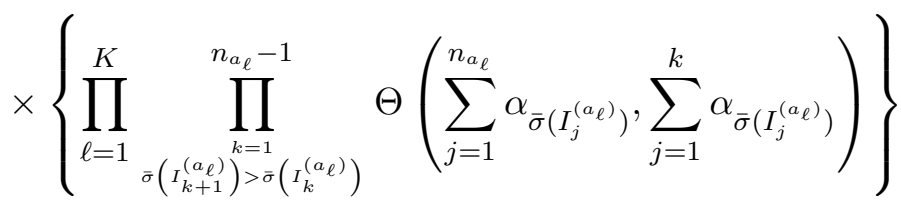

$$
\begin{aligned}
& \left.\times(-y)^{\sum_{k=1}^{K} \sum_{l^{\prime}, l \in A_{a_{k}}, l<l^{\prime}} \alpha_{\bar{\sigma}(l) \bar{\sigma}\left(l^{\prime}\right)}}(-1)^{\sum_{\ell=1}^{K} \sum_{k=1}^{n_{a_{\ell}}-1} \Theta\left(\bar{\sigma}\left(I_{k}^{\left(a_{\ell}\right)}\right)-\bar{\sigma}\left(I_{k+1}^{\left(a_{\ell}\right)}\right)\right)}\right] \\
& \times\left\{\prod_{k=1}^{K-1} \Theta\left(\alpha_{n+1}+\gamma^{\left(a_{1}\right)}+\cdots+\gamma^{\left(a_{k}\right)}, \gamma^{\left(a_{k+1}\right)}\right)\right\} \\
& \times \prod_{\ell=1}^{K}\left((-y)^{\sum_{i \in A_{a_{\ell}}} \alpha_{(n+1) i}+\sum_{r=1}^{\ell-1} \sum_{j \in A_{a_{\ell}, i \in A_{a_{r}}}} \alpha_{i j}}\right. \\
& \left.-(-y)^{-\sum_{i \in A_{a_{\ell}}} \alpha_{(n+1) i}-\sum_{r=1}^{\ell-1} \sum_{j \in A_{a_{\ell}, i \in A_{a_{r}}}} \alpha_{i j}}\right)\left(y-y^{-1}\right)^{-K}
\end{aligned}
$$

where in the expression the sum over $\bar{\sigma}$ denotes sum over a restricted set of permutations each of which permutes the elements of the set $A_{a_{k}}$ among themselves for every $k$. We shall now express the factor in the last but one line of (5.2) as

$$
\begin{aligned}
& \prod_{\ell=1}^{K}\left((-y)^{\sum_{i \in A_{\ell}} \alpha_{(n+1) i}+\sum_{r=1}^{\ell-1} \sum_{j \in A_{a_{\ell}, i \in A_{a_{r}}}} \alpha_{i j}}\right. \\
& \left.\quad-(-y)^{\sum_{i \in A_{a_{\ell}}} \alpha_{i(n+1)}+\sum_{r=1}^{\ell-1} \sum_{i \in A_{a_{\ell}, j \in A_{a_{r}}}} \alpha_{i j}}\right)
\end{aligned}
$$


and expand this as a sum of $2^{K}$ terms. After substituting this into (5.2) we get

$$
\begin{aligned}
& g_{\mathrm{KS}}\left(\alpha_{1}, \ldots, \alpha_{n+1}\right) \\
& =(-1)^{n}\left(y-y^{-1}\right)^{-n} \sum_{K=1}^{n} \sum_{\substack{\left\{a_{1}, \ldots, a_{K}\right\} \\
\gamma^{\left(a_{1}\right)}+\cdots \gamma^{\left(a_{K}\right)}=\alpha_{1}+\cdots+\alpha_{n} ; a_{1}<a_{2} \cdots<a_{K}}}(-1)^{K}
\end{aligned}
$$

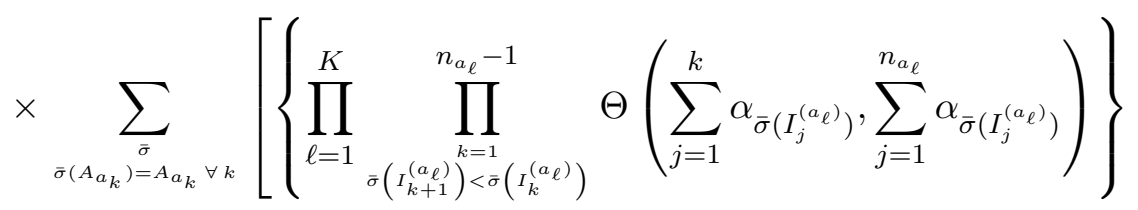

$$
\begin{aligned}
& \times\left\{\prod_{\ell=1}^{K} \prod_{\substack{k=1 \\
\bar{\sigma}\left(I_{k+1}^{\left(a_{\ell}\right)}\right)>\bar{\sigma}\left(I_{k}^{\left(a_{\ell}\right)}\right)}}^{n_{a_{\ell}}-1} \Theta\left(\sum_{j=1}^{n_{a_{\ell}}} \alpha_{\bar{\sigma}\left(I_{j}^{\left(a_{\ell}\right)}\right)}, \sum_{j=1}^{k} \alpha_{\bar{\sigma}\left(I_{j}^{\left(a_{\ell}\right)}\right)}\right)\right\} \\
& \left.\times(-y)^{\sum_{k=1}^{K} \sum_{l^{\prime}, l \in A_{a_{k}}, l<l^{\prime}} \alpha_{\bar{\sigma}(l) \bar{\sigma}\left(l^{\prime}\right)}}(-1)^{\sum_{\ell=1}^{K} \sum_{k=1}^{n_{a_{\ell}-1}} \Theta\left(\bar{\sigma}\left(I_{k}^{\left(a_{\ell}\right)}\right)-\bar{\sigma}\left(I_{k+1}^{\left(a_{\ell}\right)}\right)\right)}\right] \\
& \times \prod_{k=1}^{K-1} \Theta\left(\alpha_{n+1}+\gamma^{\left(a_{1}\right)}+\cdots+\gamma^{\left(a_{k}\right)}, \gamma^{\left(a_{k+1}\right)}\right) \\
& \times \sum_{q=0}^{K}(-1)^{K-q} \sum_{\substack{\left\{s_{1}, \ldots, s_{q}\right\} \\
1 \leq s_{1}<s_{2} \cdots<s_{q} \leq K}} \\
& \sum_{\ell \neq s_{1}, \ldots, s_{q}}\left(\sum_{i \in A_{a_{\ell}}} \alpha_{i(n+1)}+\sum_{r=1}^{\ell-1} \sum_{j \in A_{a_{r}}, i \in A_{a_{\ell}}} \alpha_{i j}\right)
\end{aligned}
$$

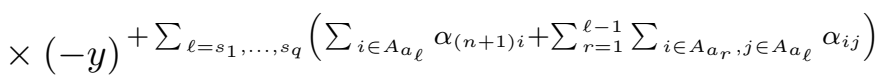

Here $s_{1}, \ldots, s_{q}$ are the values of $\ell$ in (5.3) for which we pick the first term of the factor, where for the rest of the values of $\ell$ we pick the second factor. The prefactor of $(-1)^{n}\left(y-y^{-1}\right)^{-n}$ on the right-hand side of (5.4) matches a similar factor in (2.1) with $n$ replaced by $n+1$. Leaving aside these factors the net power of $(-y)$ in a given term in the right-hand side of (5.4) 
is given by

$$
\begin{aligned}
& \sum_{k=1}^{K} \sum_{\substack{l^{\prime}, l \in A_{a_{k}} \\
l<l^{\prime}}} \alpha_{\bar{\sigma}(l) \bar{\sigma}\left(l^{\prime}\right)}+\sum_{\substack{\ell=1 \\
\ell \neq s_{1}, \ldots s_{q}}}^{K} \sum_{i \in A_{a_{\ell}}} \alpha_{i(n+1)}+\sum_{\ell=s_{1}, \ldots s_{q}} \sum_{i \in A_{a_{\ell}}} \alpha_{(n+1) i} \\
& +\sum_{\substack{\ell=1 \\
\ell \neq s_{1}, \ldots s_{q}}}^{K} \sum_{r=1}^{\ell-1} \sum_{j \in A_{a_{r}, i \in A_{a_{\ell}}}} \alpha_{i j}+\sum_{\ell=s_{1}, \ldots s_{q}} \sum_{r=1}^{\ell-1} \sum_{i \in A_{a_{r}}, j \in A_{a_{\ell}}} \alpha_{i j} .
\end{aligned}
$$

This can be expressed as

$$
\sum_{\substack{i, j=1 \\ i<j}}^{n+1} \alpha_{\sigma(i) \sigma(j)}
$$

where $\sigma$ denotes a permutation of $\{1, \ldots, n+1\}$ given by

$$
\begin{aligned}
\{\sigma(1), \ldots, \sigma(n+1)\}= & \left\{\bar{\sigma}\left(A_{a_{K}}\right), \ldots, \bar{\sigma}\left(\not_{a_{s_{q}}}\right), \cdots, \bar{\sigma}\left(\not A_{a_{s_{1}}}\right) \cdots \bar{\sigma}\left(A_{a_{1}}\right)\right. \\
& \left.n+1, \bar{\sigma}\left(A_{a_{s_{1}}}\right), \ldots, \bar{\sigma}\left(A_{a_{s_{q}}}\right)\right\}
\end{aligned}
$$

$\bar{\sigma}\left(A_{a_{\ell}}\right)$ contains the elements of $A_{a_{\ell}}$ ordered according to the permutation $\bar{\sigma}$ restricted to the set $A_{a_{\ell}}$. The symbol $\bar{\sigma}\left(A_{a_{\ell}}\right)$ denotes that the corresponding $\bar{\sigma}\left(A_{a_{\ell}}\right)$ is missing from the list, since it is placed on the right-hand side of $n+1$.

We shall now try to reorganize the sum in (5.4) by first summing over all contributions corresponding to a given permutation $\sigma$ of $(1, \ldots, n+1)$, and then summing over $\sigma$. Let $R$ denote the position of $n+1$ on the right-hand side of (5.7), i.e., $\sigma(R)=n+1$. We introduce sets of integers

$B_{1}=\left\{1, \ldots, I_{1}-1\right\}, B_{2}=\left\{I_{1}, \ldots, I_{2}-1\right\}, \cdots, B_{p}=\left\{I_{p-1}, \ldots,(R-1)\right\}$,

and

$$
\begin{aligned}
& C_{q}=\left\{(R+1), \ldots, J_{1}-1\right\}, \quad C_{q-1}=\left\{J_{1}, \ldots, J_{2}-1\right\}, \cdots, \\
& C_{1}=\left\{J_{q-1}, \ldots,(n+1)\right\} .
\end{aligned}
$$


for appropriate integers $I_{1}, \ldots I_{p-1}$ and $J_{1}, \ldots J_{q-1}$ with $p=K-q$ and $I_{1}<$ $I_{2}<\cdots I_{p-1}<R<J_{1}<J_{2}<\cdots<J_{q-1}$ such that

$$
\begin{aligned}
\left\{\left\{\sigma\left(B_{1}\right)\right\}, \ldots,\left\{\sigma\left(B_{p}\right)\right\}\right\}= & \left\{\left\{\bar{\sigma}\left(A_{a_{K}}\right)\right\}, \ldots,\left\{\bar{\sigma}\left(A_{a_{s_{q}}}\right)\right\}, \cdots,\right. \\
& \left.\times\left\{\bar{\sigma}\left(\not_{a_{s_{1}}}\right)\right\} \cdots\left\{\bar{\sigma}\left(A_{a_{1}}\right)\right\}\right\} \\
\left\{\left\{\sigma\left(C_{q}\right)\right\}, \cdots\left\{\sigma\left(C_{1}\right)\right\}\right\}= & \left\{\left\{\bar{\sigma}\left(A_{a_{s_{1}}}\right)\right\}, \cdots,\left\{\bar{\sigma}\left(A_{a_{s_{q}}}\right)\right\}\right\},
\end{aligned}
$$

as ordered sets. Thus $\sigma\left(B_{a}\right)$ 's correspond to the sets of $\bar{\sigma}\left(A_{a_{i}}\right)$ 's in (5.7) to the left of $(n+1)$ and $\sigma\left(C_{a}\right)$ 's correspond to the sets of $\bar{\sigma}\left(A_{a_{i}}\right)$ 's in (5.7) to the right of $(n+1)$. We also define: ${ }^{4}$

$$
\begin{aligned}
\delta^{(a)}=\sum_{i \in B_{a}} \alpha_{\sigma(i)}= \begin{cases}\sum_{i=1}^{I_{1}-1} \alpha_{\sigma(i)} & \text { for } \quad a=1, \\
\sum_{i=I_{a-1}}^{I_{a}-1} \alpha_{\sigma(i)} & \text { for } \quad p-1 \geq a \geq 2, \\
\sum_{i=I_{p-1}}^{R-1} \alpha_{\sigma(i)} & \text { for } \quad a=p,\end{cases} \\
\tau_{i \in C_{a}}^{(a)} \alpha_{\sigma(i)}= \begin{cases}\sum_{i=R+1}^{J_{1}-1} \alpha_{\sigma(i)} & \text { for } a=q, \\
\sum_{i=J_{q-a}} \alpha_{\sigma(i)} & \text { for } \quad q-1 \geq a \geq 2, \\
\sum_{i=J_{q-1}}^{n+1} \alpha_{\sigma(i)} & \text { for } a=1 .\end{cases}
\end{aligned}
$$

The $\delta^{(a)}$ 's correspond to the $\gamma^{\left(a_{i}\right)}$ 's for $i=K, K-1, \ldots, \phi_{q}, \ldots, \phi_{1}, \ldots 1$ and $\tau^{(a)}$ 's correspond to the $\gamma^{\left(a_{i}\right)}$ 's for $i=s_{q}, s_{q-1}, \ldots, s_{1}$ in (5.4). A pictorial

\footnotetext{
${ }^{4}$ We shall use the same index $a$ for $B_{a}, C_{a}, \delta^{(a)}$ and $\tau^{(a)}$. However it should be understood that for $B_{a}$ and $\delta^{(a)}$ the index runs from 1 to $p$ and for $C_{a}$ and $\tau^{(a)}$ the index runs from 1 to $q$.
} 
representation of this arrangement can be given as follows:

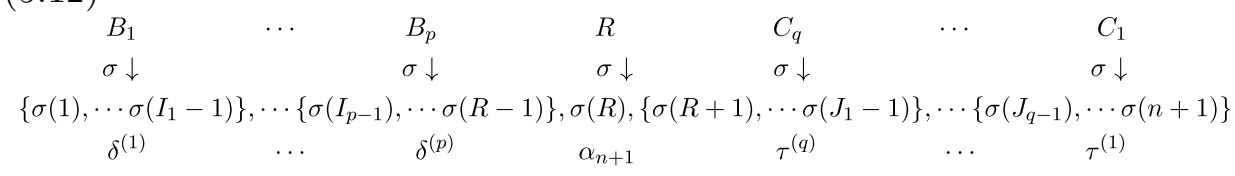

The last row describes the sum of all the $\alpha_{\sigma(i)}$ 's in the sets in the last but one row. The partitioning described above must satisfy the following constraints:

(1) The restrictions $a_{1}<a_{2}<\cdots a_{K}$ and $s_{1}<s_{2}<\cdots s_{q}$ in (5.4) translate to the following restrictions on $\left\{\delta^{(a)}\right\},\left\{\tau^{(a)}\right\}$ :

$$
\delta^{(1)}>\delta^{(2)}>\cdots>\delta^{(p)}, \quad \tau^{(1)}>\tau^{(2)}>\cdots>\tau^{(q)} .
$$

(2) Let us denote by $\widehat{\gamma}^{(k)}(1 \leq k \leq p+q)$ the set of vectors $\left\{\delta^{(1)}, \ldots \delta^{(p)}\right.$, $\left.\tau^{(1)}, \ldots \tau^{(q)}\right\}$ ordered so that $\widehat{\gamma}^{(1)}<\widehat{\gamma}^{(2)}<\ldots \widehat{\gamma}^{(p+q)}$. Thus we have $\widehat{\gamma}^{(k)}=\gamma^{\left(a_{k}\right)}$ and the third set of $\Theta$ 's in (5.4) imposes the constraints:

$$
\alpha_{n+1}+\widehat{\gamma}^{(1)}+\cdots+\widehat{\gamma}^{(k)}>\widehat{\gamma}^{(k+1)} \quad \text { for } \quad k=1,2, \ldots, p+q-1 .
$$

Using (1.6) this is equivalent to the condition

$$
\begin{aligned}
& \alpha_{n+1}+\widehat{\gamma}^{(1)}>\alpha_{n+1}+\widehat{\gamma}^{(1)}+\widehat{\gamma}^{(2)}>\cdots>\alpha_{n+1} \\
& +\widehat{\gamma}^{(1)}+\widehat{\gamma}^{(2)}+\cdots+\widehat{\gamma}^{(p+q)}=\bar{\alpha} .
\end{aligned}
$$

(3) Since for $I_{a-1} \leq k \leq I_{a}-1, k \in B_{a}$, which is one of the $A_{a_{i}}$ 's appearing in (5.4), the first and second sets of $\Theta$ 's in (5.4) impose the constraints:

$$
\begin{gathered}
\delta^{(a)}>\sum_{i=I_{a-1}}^{k} \alpha_{\sigma(i)} \quad \text { for } \quad \sigma(k+1)>\sigma(k), \\
\delta^{(a)}<\sum_{i=I_{a-1}}^{k} \alpha_{\sigma(i)} \quad \text { for } \quad \sigma(k+1)<\sigma(k), \\
I_{a-1} \leq k \leq I_{a}-2, \quad 1 \leq a \leq p, \quad I_{0} \equiv 1, \quad I_{p} \equiv R .
\end{gathered}
$$

(4) Similarly since for $J_{a-1} \leq k \leq J_{a}-1, k \in C_{q-a+1}$, which is one of the $A_{a}$ 's appearing in (5.4), the first and second sets of $\Theta$ 's in (5.4) impose 
the constraints:

$$
\begin{aligned}
& \tau^{(q+1-a)}>\sum_{j=J_{a-1}}^{k} \alpha_{\sigma(j)} \quad \text { for } \sigma(k+1)>\sigma(k), \\
& \tau^{(q+1-a)}<\sum_{j=J_{a-1}}^{k} \alpha_{\sigma(j)} \quad \text { for } \quad \sigma(k+1)<\sigma(k), \\
& J_{a-1} \leq k \leq J_{a}-2, \quad 1 \leq a \leq q, \quad J_{0} \equiv R+1, \quad J_{q} \equiv n+2 .
\end{aligned}
$$

Note that under the reversal of the permutation associated with $\sigma$ the roles of $\tau^{(a)}$ and $\delta^{(a)}$ get interchanged.

We can now run the arguments in reverse to find an algorithm for computing $g_{\mathrm{KS}}$ as a sum over permutations and partitions. Given any permutation $\sigma$, we consider all possible choices of the sets $B_{a}$ and $C_{a}$ encoded in the choice of the integers $p, q, I_{1}, \ldots, I_{p-1}, J_{1}, \ldots, J_{q-1}$. It is easy to see that for a given choice of $\sigma$ and the integers $p, q, I_{1}, \ldots, I_{p-1}, J_{1}, \ldots, J_{q-1}$, the summation variable $K=p+q$ and permutations $\bar{\sigma}$ in (5.4) are completely fixed. We then need to verify if the corresponding $\left\{\delta^{(a)}\right\}$ and $\left\{\tau^{(a)}\right\}$ satisfy the four conditions mentioned above. If they do then we shall call this choice of $\left\{p, q, I_{1}, \ldots, I_{p-1}, J_{1}, \ldots, J_{q-1}\right\}$ an allowed partition. The net contribution for a given $\sigma$ is then obtained by summing over all the allowed partitions weighted by the factors which appear in (5.4). With the help of (5.6), the contribution to $g_{\mathrm{KS}}\left(\alpha_{1}, \ldots, \alpha_{n+1}\right)$ given in (5.4) may then be written as

$g_{\mathrm{KS}}\left(\alpha_{1}, \ldots, \alpha_{n+1}\right)=(-1)^{n}\left(y-y^{-1}\right)^{-n} \sum_{\sigma} \mathrm{N}_{\mathrm{KS}}^{(n+1)}\left(\left\{\alpha_{i}\right\} ; \sigma\right)(-y)^{\sum_{l<k} \alpha_{\sigma(l) \sigma(k)}}$,

where

$\mathrm{N}_{\mathrm{KS}}^{(n+1)}\left(\left\{\alpha_{i}\right\} ; \sigma\right)=\sum_{\text {allowed partitions }}(-1)^{q+\sum_{a=1}^{p} k_{a}+\sum_{a=1}^{q} l_{a}}$,

$k_{a} \equiv \sum_{k \in B_{a}, k+1 \in B_{a}} \Theta(\sigma(k)-\sigma(k+1)), l_{a} \equiv \sum_{k \in C_{a}, k+1 \in C_{a}} \Theta(\sigma(k)-\sigma(k+1))$. 
Our goal will be to show that $\mathrm{N}_{\mathrm{KS}}^{(n+1)}\left(\left\{\alpha_{i}\right\} ; \sigma\right)$ defined this way agrees with the coefficient $\mathrm{N}_{\text {higgs }}^{(n+1)}\left(\left\{\alpha_{i}\right\} ; \sigma\right)$ given in $(2.1)$.

\subsection{Deforming the $\alpha_{i}$ 's}

As mentioned in Section 1, we have taken the $\alpha_{i}$ 's to be generic so that they have finite length and the angle between two vectors of the form $\sum_{i \in S_{1}} \alpha_{i}$ and $\sum_{i \in S_{2}} \alpha_{i}$ for any pair of non-overlapping sets $S_{1}, S_{2}$ is non-vanishing. We have also seen that neither the result for $\mathrm{N}_{\mathrm{KS}}^{(n+1)}\left(\left\{\alpha_{i}\right\} ; \sigma\right)$ nor the result for $\mathrm{N}_{\text {higgs }}^{(n+1)}\left(\left\{\alpha_{i}\right\} ; \sigma\right)$ changes under a deformation of the $\alpha_{i}$ 's which preserves the relative orientation of the $\alpha_{i}$ 's and the relative orientation of $\sum_{i=1}^{k} \alpha_{\sigma(i)}$ with respect to $\sum_{i=1}^{n+1} \alpha_{i}$ for all $k$. Our strategy now will be to use the freedom to deform the $\alpha_{i}$ 's and by this process bring some of the angles and lengths arbitrarily close to zero - much smaller than the angles and lengths in the starting configuration. Since the angles and lengths, which we have brought arbitrarily close to zero are now much smaller than the other angles and lengths, which we do not change during the deformation - which we shall refer to as generic lengths and angles - the computation of $\mathrm{N}_{\mathrm{KS}}^{(n+1)}\left(\left\{\alpha_{i}\right\} ; \sigma\right)$ given in (5.19) will simplify in this new configuration. We shall then compare this $\mathrm{N}_{\mathrm{KS}}^{(n+1)}\left(\left\{\alpha_{i}\right\} ; \sigma\right)$ to $\mathrm{N}_{\text {higgs }}^{(n+1)}\left(\left\{\alpha_{i}\right\} ; \sigma\right)$ obtained by replacing $n$ by $n+1$ in (2.1).

For notational simplicity it will be convenient to define the following quantities associated with a given permutation $\sigma$ :

$$
\widetilde{\alpha}_{i}=\alpha_{\sigma(i)}, \quad \bar{\alpha}=\sum_{j=1}^{n+1} \alpha_{j}=\sum_{j=1}^{n+1} \widetilde{\alpha}_{j}, \quad \beta_{k}=\sum_{i=1}^{k} \widetilde{\alpha}_{i}, \quad \text { for } 1 \leq i, k \leq n+1 .
$$

In this notation, we have the ordering

$$
\widetilde{\alpha}_{i}>\widetilde{\alpha}_{j} \text { if } \sigma(i)>\sigma(j) \Rightarrow \Theta\left(\widetilde{\alpha}_{i}, \widetilde{\alpha}_{j}\right)=\Theta(\sigma(i)-\sigma(j)) .
$$

The allowed deformations are those which preserve the relative ordering of the $\widetilde{\alpha}_{i}$ 's and the relative ordering between $\beta_{k}$ and $\bar{\alpha}$ for each $k$.

Now if we deform all the $\widetilde{\alpha}_{i}$ 's at once, or even a pair of $\widetilde{\alpha}_{i}$ 's which are not placed next to each other in the chain $\left\{\widetilde{\alpha}_{1}, \ldots, \widetilde{\alpha}_{n+1}\right\}$, it will change many $\beta_{i}$ 's at once, and we need to ensure that none of these $\beta_{i}$ 's cross over from one side of $\bar{\alpha}$ to the other side. For this reason we shall deform the $\alpha_{i}$ 's in 
nearest neighbor pairs: take a pair $\left(\widetilde{\alpha}_{j}, \widetilde{\alpha}_{j+1}\right)$ and deform it to

$$
\left(\widetilde{\alpha}_{j}+\lambda \widetilde{\alpha}_{j}, \widetilde{\alpha}_{j+1}+\lambda^{\prime} \widetilde{\alpha}_{j+1}\right)
$$

with $\left(\lambda, \lambda^{\prime}\right)$ a pair of real numbers satisfying the following conditions:

(1) $\lambda, \lambda^{\prime}>-1$.

(2) $\lambda \widetilde{\alpha}_{j}+\lambda^{\prime} \widetilde{\alpha}_{j+1} \propto \bar{\alpha}$. This condition determines $\lambda^{\prime}$ in terms of $\lambda$ and makes this into a one parameter deformation.

(3) At least one of $\lambda$ or $\lambda^{\prime}$ is negative. We can for definiteness take $\lambda$ to be negative.

Clearly (5.23) and the first condition above ensures that in the new configuration $\widetilde{\alpha}_{j}, \widetilde{\alpha}_{j+1}$ preserve their directions. The first and the second condition ensure that with the new $\widetilde{\alpha}_{i}$ 's, the new $\bar{\alpha}$ remains parallel to the original $\bar{\alpha}$ and continues to be directed along the first quadrant. Finally, the third condition ensures that at least one of the vectors among $\widetilde{\alpha}_{j}, \widetilde{\alpha}_{j+1}$ reduces its length during this deformation. We have taken this to be the vector $\widetilde{\alpha}_{j}$.

Now during this deformation all the $\beta_{k}$ 's for $k<j$ are preserved, while we add a vector $\lambda \widetilde{\alpha}_{j}+\lambda^{\prime} \widetilde{\alpha}_{j+1} \propto \bar{\alpha}$ to the $\beta_{k}$ 's for $k \geq j+1$. Thus, the $\beta_{k}$ 's for $k<j$ and $k>j$ cannot cross $\bar{\alpha}$, and as long as $\beta_{j}$ does not cross the vector $\bar{\alpha}$, this map preserves $\mathrm{N}_{\mathrm{KS}}^{(n+1)}$ and $\mathrm{N}_{\text {higgs }}^{(n+1)}$. We can increase the magnitude of the deformation till one of the following situation is encountered: ${ }^{5}$

(1) The orientation of $\beta_{j}$ may approach that of $\bar{\alpha}$. In order to preserve $N_{\mathrm{KS}}^{(n+1)}$ and $N_{\text {higgs }}^{(n+1)}$, we must stop the deformation infinitesimally before $\beta_{j}$ becomes exactly parallel to $\bar{\alpha}$. In this case we shall say that $\beta_{j}$ has become almost parallel to $\bar{\alpha}$.

(2) $\widetilde{\alpha}_{j}$ may approach zero. In this case $\beta_{j}=\beta_{j-1}+\widetilde{\alpha}_{j} \rightarrow \beta_{j-1}$. Thus, such a situation can arise before we encounter the first possibility only if $\beta_{j}$ and $\beta_{j-1}$ were on the same side of $\bar{\alpha}$ to begin with. As before we need to stop the deformation infinitesimally before $\widetilde{\alpha}_{j}$ becomes exactly zero. In this case, we shall say that $\widetilde{\alpha}_{j}$ has been made almost zero.

(3) $\widetilde{\alpha}_{j+1}$ may approach zero. In this case $\beta_{j}=\beta_{j+1}-\widetilde{\alpha}_{j+1} \rightarrow \beta_{j+1}$ and hence such a situation can arise before encountering the first case only

\footnotetext{
${ }^{5}$ Since our initial choice of vectors were generic we need not consider the case where two of these events occur simultaneously except when it is occurs as a result of some identity that holds for generic $\left\{\alpha_{i}\right\}$ 's.
} 
if $\beta_{j}$ and $\beta_{j+1}$ were on the same side of $\bar{\alpha}$ to begin with. In this case, we shall say that $\widetilde{\alpha}_{j+1}$ has been made almost zero.

If $\beta_{j}$ becomes almost parallel to $\bar{\alpha}$ first we stop the process here. Otherwise we can continue the process as follows. If we have made $\widetilde{\alpha}_{j}$ almost zero then we can repeat the process with the pair $\left(\widetilde{\alpha}_{j-1}, \widetilde{\alpha}_{j+1}\right)$. The deformation will now affect both $\beta_{j-1}$ and $\beta_{j}$, but $\beta_{j}=\beta_{j-1}+\widetilde{\alpha}_{j}$ is now almost equal to $\beta_{j-1}$, and as long as we ensure that the deformation does not take $\beta_{j-1}$ across $\bar{\alpha}, \beta_{j}$ also does not cross $\bar{\alpha}$. Similarly if $\widetilde{\alpha}_{j+1}$ has been made almost zero, we can continue the analysis with the pair $\left(\widetilde{\alpha}_{j}, \widetilde{\alpha}_{j+2}\right)$. Repeating this procedure we see that at any stage we work with a pair $\left(\widetilde{\alpha}_{k}, \widetilde{\alpha}_{\ell}\right)(k \leq j<\ell)$ with all the intervening $\widetilde{\alpha}_{i}$ 's zero. This process stops when $\beta_{k}$ becomes almost parallel to $\bar{\alpha}$. Once this happens, all the other $\beta_{i}$ 's for $k<i<\ell$ (including the $\beta_{j}$ associated with the starting position) also become almost parallel to $\bar{\alpha}$ since the corresponding $\widetilde{\alpha}_{i}$ 's have already been made almost zero. We note furthermore that by our previous argument (points 2 and 3 above) the chain cannot continue past a point $k_{0}$ for which $\beta_{k_{0}-1}$ and $\beta_{k_{0}}$ are on the opposite sides of $\bar{\alpha}$. The situation can be represented as

$$
\left(\begin{array}{lllllllll}
\operatorname{position} i & & k & k+1 & k+2 & \cdots & \ell-2 & \ell-1 & \ell \\
\widetilde{\alpha}_{i} & \simeq & \cdot & 0 & 0 & \cdots & 0 & 0 & . \\
\beta_{i} & \imath \| & \bar{\alpha} & \bar{\alpha} & \bar{\alpha} & \cdots & \bar{\alpha} & \bar{\alpha} & \cdot
\end{array}\right)
$$

with all the $\beta_{i}$ 's for $k \leq i<\ell$ being on the same side of $\bar{\alpha}$. Here the symbol $2 \|$ denotes "almost parallel to". The only exception to this is when in the above diagram $k=1$ or $\ell-1=n+1$. Such a situation can arise if in the starting configuration all the $\beta_{i}$ 's for $i \leq j$ were on the same side of $\bar{\alpha}$ or all the $\beta_{i}$ 's for $i \geq j$ were on the same side of $\bar{\alpha}$. In the former case we may arrive at a configuration in which all the $\widetilde{\alpha}_{i}$ 's and $\beta_{i}$ 's for $1 \leq i<\ell$ are almost zero but none of the $\beta_{i}$ 's are almost parallel to $\bar{\alpha}$. In the latter case, we can arrive at a situation where all the $\widetilde{\alpha}_{i}$ 's for $k<i \leq n+1$ are almost zero and all the $\beta_{i}$ 's for $k \leq i \leq n$ are almost equal (and hence almost parallel) to $\bar{\alpha}$ since by definition $\beta_{n+1}=\bar{\alpha}$.

In what follows, the neighborhood of the location of $\alpha_{n+1}$ will play a special role. We shall denote the position of $\alpha_{n+1}$ by $R$, i.e., $\widetilde{\alpha}_{R}=\alpha_{n+1}$. Thus $R$ marks the maximum of $\sigma(i)$. We shall carry out the manipulation described above by taking our starting pair to be $(R-1, R)$. Except for the special cases mentioned in the last paragraph, which will be discussed separately later, at the end of the manipulation we shall arrive at a situation where $\beta_{R-1}$ and possibly some other $\beta_{i}$ 's around it have been made almost parallel to $\bar{\alpha}$, and some of the $\widetilde{\alpha}_{i}$ 's around $R$ have been made almost zero. 
If the set of points where $\widetilde{\alpha}_{i}$ becomes almost zero includes also the point $R$, then we do not carry out any further deformation of this system. If on the other hand it does not extend beyond $R-1$ (e.g., for the case when $\beta_{R-1}$ and $\beta_{R}$ are on the opposite sides of $\left.\bar{\alpha}\right)$ then we start with the pair $(R, R+1)$ and carry out a similar manipulation. At the end of this process we can have several situations:

(1) Generically we would get a configuration in which we have

$$
\left(\begin{array}{llllllllllllll}
\text { position } & & P-1 & P & P+1 & P+2 & \ldots & R-1 & R & \cdots & Q-2 & Q-1 & Q \\
\tilde{\alpha}_{i} & \simeq & . & & & 0 & 0 & \cdots & 0 & 0 & \cdots & 0 & 0 & . \\
\beta_{i} & 2 \| & . & & \bar{\alpha} & \bar{\alpha} & \bar{\alpha} & \ldots & \bar{\alpha} & \bar{\alpha} & \cdots & \bar{\alpha} & \bar{\alpha} & \cdot
\end{array}\right),
$$

for some positions $R>P>1$ and $R<Q<n+1$ in the chain. Furthermore all the $\beta_{i}$ 's for $P \leq i \leq R-1$ must be on the same side of $\bar{\alpha}$ and all the $\beta_{i}$ 's for $R \leq i \leq Q-1$ must be on the same side of $\bar{\alpha}$. Whether these two sets of $\beta_{i}$ 's lie on the same side of $\bar{\alpha}$ or not depends on whether in the initial configuration $\beta_{R-1}$ and $\beta_{R}$ lie on the same side of $\bar{\alpha}$ or not.

(2) If in the starting configuration all the $\beta_{i}$ 's for $i \leq R-1$ are on the same side of $\bar{\alpha}$ then the chain may continue all the way to the left, setting all the $\widetilde{\alpha}_{i}$ 's for $i \leq R-1$ to almost zero. Similarly if all the $\beta_{i}$ 's for $i \geq R$ are on the same side of $\bar{\alpha}$ then the chain may continue all the way to the right setting $\widetilde{\alpha}_{i}$ to be almost zero for all $i>R$. We shall consider these special cases separately.

It is useful to note that under a reversal of permutation, the role of $\beta$ is played by $\sum_{i=j}^{n+1} \widetilde{\alpha}_{i}=\bar{\alpha}-\beta_{j-1}$. Thus, to see the role of reversing the permutation we can express (5.25) as

$$
\left(\begin{array}{llllllllllllll}
\text { position } & & Q & Q-1 & Q-2 & Q-3 & \ldots & R & R-1 & \ldots & P+1 & P & P-1 \\
\tilde{\alpha}_{i} & \simeq & . & 0 & 0 & 0 & \ldots & 0 & 0 & \ldots & 0 & . & . \\
\bar{\alpha}-\beta_{i-1} & 2 \| & \bar{\alpha} & \bar{\alpha} & \bar{\alpha} & \bar{\alpha} & \ldots & \bar{\alpha} & \bar{\alpha} & \ldots & \bar{\alpha} & . & .
\end{array}\right) .
$$

Comparing (5.25) and (5.26) we see that the roles of the points $P$ and $Q$ are exchanged under a reversal of permutation. 


\subsection{Constraining the permutations and partitions}

We shall now show that by making use of the deformations described in Section 5.2 we can put severe restrictions on the permutations $\sigma$, as well as the choices of the sets $\left\{B_{a}\right\},\left\{C_{a}\right\}$, which can contribute to $\mathrm{N}_{\mathrm{KS}}^{(n+1)}\left(\left\{\alpha_{i}\right\} ; \sigma\right)$. In particular, we shall show that

(1) $\mathrm{N}_{\mathrm{KS}}^{(n+1)}\left(\left\{\alpha_{i}\right\} ; \sigma\right)$ vanishes unless

$$
\beta_{R-1}<\bar{\alpha}<\beta_{R}
$$

Combining this with the previous results we can also conclude that we must have

$$
\beta_{i}<\bar{\alpha} \quad \text { for } P \leq i \leq R-1, \quad \beta_{i}>\bar{\alpha} \quad \text { for } R \leq i<Q
$$

(2) For a configuration satisfying (5.27) the choice of the sets $B_{1}$ and $C_{1}$ described in (5.8), (5.9) must be such that

$$
I_{1} \geq P+1, \quad J_{q-1} \leq Q
$$

Thus $B_{1}$ should include at least the elements $(1,2, \ldots, P)$ and $C_{1}$ should include at least the elements $(Q, Q+1, \ldots, n+1)$.

(3) In order that a permutation contributes to $\mathrm{N}_{\mathrm{KS}}^{(n+1)}$, we must have

$$
\begin{aligned}
& \beta_{k}\left\{\begin{array}{ll}
<\bar{\alpha} & \text { for } \sigma(k+1)>\sigma(k), \\
>\bar{\alpha} & \text { for } \sigma(k+1)<\sigma(k),
\end{array} \quad 1 \leq k<P,\right. \\
& \beta_{k}\left\{\begin{array}{ll}
<\bar{\alpha} & \text { for } \sigma(k+1)>\sigma(k), \\
>\bar{\alpha} & \text { for } \sigma(k+1)<\sigma(k),
\end{array} \quad Q \leq k<n+1 .\right.
\end{aligned}
$$

Proof of (5.27): If $\beta_{R-1}>\bar{\alpha}$ then clearly $\beta_{R}=\beta_{R-1}+\widetilde{\alpha}_{R}=\beta_{R-1}+$ $\alpha_{n+1}>\bar{\alpha}$. Thus, to prove (5.27) we only have to show that $\mathrm{N}_{\mathrm{KS}}^{(n+1)}$ vanishes when $\beta_{R}, \beta_{R-1}>\bar{\alpha}$ or $\beta_{R}, \beta_{R-1}<\bar{\alpha}$. These two cases are in fact related by permutation reversal, so it is enough to consider one of them. We shall consider the case $\beta_{R-1}, \beta_{R}<\bar{\alpha}$.

We proceed as in Section 5.2, reducing $\widetilde{\alpha}_{i}$ 's for $i<R$ one by one by starting with the pair $(R-1, R)$. As we have seen, when $\beta_{P}$ is almost parallel to $\bar{\alpha}$ with all the $\widetilde{\alpha}_{i}$ for $P<i \leq R-1$ almost zero, then all $\beta_{k}$ for $P \leq k \leq$ $R-1$ become almost parallel to $\bar{\alpha}$. In particular, we have $\beta_{R-1} \simeq c \bar{\alpha}$ for 
some positive constant $c$. If at this stage $\widetilde{\alpha}_{R}=\alpha_{n+1}$ has finite length, then we shall have $\beta_{R}=\beta_{R-1}+\widetilde{\alpha}_{R} \simeq c \bar{\alpha}+\alpha_{n-1}>\bar{\alpha}$, contradicting our assumption that the starting configuration has $\beta_{R}<\bar{\alpha}$. This shows that before we reach the stage where $\beta_{P}$ becomes almost parallel to $\bar{\alpha}, \widetilde{\alpha}_{R}=\alpha_{n+1}$ should become almost zero. Let us stop the deformation as soon as $\widetilde{\alpha}_{R}$ becomes almost zero and try to check if the required conditions are satisfied by any choice of the sets $\left\{B_{a}\right\},\left\{C_{a}\right\}$. Since we have stopped the deformation at a stage where some of the $\widetilde{\alpha}_{i}$ 's may be almost zero but none of the $\beta_{i}$ 's are almost parallel to $\bar{\alpha}$, at least $\delta^{(1)}$ and $\tau^{(1)}$, which can be identified as $\beta_{I_{1}-1}$ and $\bar{\alpha}-\beta_{J_{q-1}-1}$, remain generic. Now (5.13) shows that when we order the $\delta^{(a)}$ 's and $\tau^{(a)}$ 's into the sets $\widehat{\gamma}^{(k)}$ with $\widehat{\gamma}^{(1)}, \ldots, \widehat{\gamma}^{(p+q)}$ in the order $\widehat{\gamma}^{(1)}<\widehat{\gamma}^{(2)}<\cdots<\widehat{\gamma}^{(p+q)}$, then $\widehat{\gamma}^{(p+q)}$ must be either $\delta^{(1)}$ or $\tau^{(1)}$ depending on whether $\delta^{(1)}>\tau^{(1)}$ or $\tau^{(1)}>\delta^{(1)}$. Thus $\widehat{\gamma}^{(p+q)}$ and hence also $\bar{\alpha}-\widehat{\gamma}^{(p+q)}=\alpha_{n+1}+\widehat{\gamma}^{(1)}+\widehat{\gamma}^{(2)}+$ $\cdots+\widehat{\gamma}^{(p+q-1)}$ are generic, i.e., neither almost zero nor almost parallel to $\bar{\alpha}$. In this case, for $k=p+q-1$ we can drop the $\alpha_{n+1}$ from the left-hand side of (5.14) since it has been made almost zero, and express (5.14) as

$$
\widehat{\gamma}^{(1)}+\cdots \widehat{\gamma}^{(p+q-1)}>\widehat{\gamma}^{(p+q)} \text {. }
$$

This is in obvious contradiction to the fact that $\widehat{\gamma}^{(k)}$ 's are ordered as

$$
\widehat{\gamma}^{(1)}<\widehat{\gamma}^{(2)}<\cdots<\widehat{\gamma}^{(p+q)}
$$

Thus, we see that there is no choice of the sets $\left\{B_{a}\right\},\left\{C_{a}\right\}$ satisfying the necessary conditions. This shows that unless $(5.27)$ holds, $\mathrm{N}_{\mathrm{KS}}^{(n+1)}\left(\left\{\alpha_{i}\right\} ; \sigma\right)$ will vanish.

Proof of (5.29): We shall now examine, for a configuration satisfying (5.27), possible ways of dividing the set to the left of $R$ into the sets $\left\{B_{a}\right\}$ and the set to the right of $R$ into the sets $\left\{C_{a}\right\}$ subject to the constraints given in (5.13) to (5.17). For this we shall carry out the deformations all the way to the end so that the final configuration at the end of the deformation takes the form (5.25). Now it follows from the ordering $\widehat{\gamma}^{(1)}<\widehat{\gamma}^{(2)}<\cdots<\widehat{\gamma}^{(p+q)}$ that for any $k$ we have

$$
\widehat{\gamma}^{(1)}+\cdots \widehat{\gamma}^{(k)}<\widehat{\gamma}^{(k+1)}+\widehat{\gamma}^{(k+2)}+\cdots+\widehat{\gamma}^{(p+q)} .
$$

On the other hand, it follows from (5.15) that

$$
\begin{aligned}
& \alpha_{n+1}+\widehat{\gamma}^{(1)}+\cdots+\widehat{\gamma}^{(k)}>\bar{\alpha}-\left(\alpha_{n+1}+\widehat{\gamma}^{(1)}+\cdots+\widehat{\gamma}^{(k)}\right) \\
& =\widehat{\gamma}^{(k+1)}+\widehat{\gamma}^{(k+2)}+\cdots+\widehat{\gamma}^{(p+q)} .
\end{aligned}
$$


Let $k_{0}$ be the minimum value of $k$ for which $\widehat{\gamma}^{\left(k_{0}\right)}$ is generic, i.e., neither almost zero nor almost parallel to $\bar{\alpha}$. In this case for $k=k_{0}$ we can drop the $\alpha_{n+1}$ from the left-hand side of (5.35) since $\alpha_{n+1}$ has been made almost zero, and express it as

$$
\widehat{\gamma}^{(1)}+\cdots+\widehat{\gamma}^{\left(k_{0}\right)}>\widehat{\gamma}^{\left(k_{0}+1\right)}+\widehat{\gamma}^{\left(k_{0}+2\right)}+\cdots+\widehat{\gamma}^{(p+q)} .
$$

This is in obvious contradiction to (5.34) for $k=k_{0}$ showing that our initial assumption must be wrong. In other words all the $\widehat{\gamma}^{(k)}$ 's must be either almost zero or almost parallel to $\bar{\alpha}$. Since the set $\left\{\widehat{\gamma}^{(k)}\right\}$ includes $\delta^{(1)}=$ $\sum_{i=1}^{I_{1}-1} \widetilde{\alpha}_{i}$ and $\tau^{(1)}=\sum_{i=J_{q-1}}^{n+1} \widetilde{\alpha}_{i}$, they must also satisfy this criterion. This can happen only if $I_{1}>P$ and $J_{q-1} \leq Q$ since otherwise $\delta^{(1)}$ and/or $\tau^{(1)}$ will involve a sum of $\widetilde{\alpha}_{i}$ 's which have not been deformed and hence must be generic. Thus we must satisfy (5.29), and as a consequence $\delta^{(1)}$ and $\tau^{(1)}$ are almost parallel to $\bar{\alpha}$.

Proof of (5.30), (5.31): For this we first test (5.16) for $a=1$. Since $I_{0}=1$, we have $\sum_{i=I_{0}}^{k} \alpha_{\sigma(i)}=\beta_{k}$ for $k \in B_{1}$. Since for $i<P$, we have not deformed $\widetilde{\alpha}_{i}$ 's and $\beta_{i}$ 's, they are generic. On the other hand as argued above $\delta^{(1)}$ appearing in (5.16) for $a=1$ is almost parallel to $\bar{\alpha}$. Hence in testing (5.16) for $a=1$ and $k<P$, replacing $\delta^{(1)}$ by $\bar{\alpha}$ does not make any difference. After making these replacements we get $\beta_{k}<\bar{\alpha}$ for $\sigma(k+1)>\sigma(k)$ and $\beta_{k}>\bar{\alpha}$ for $\sigma(k+1)<\sigma(k)$ for $1 \leq k<P$. This gives (5.30). Similarly testing (5.17) for $a=q$ we get (5.31) since this is related to the previous case by a reversal of permutation.

\subsection{Comparison with the constraints on $\sigma$ for non-vanishing $\mathrm{N}_{\text {higgs }}^{(n+1)}\left(\left\{\alpha_{i}\right\} ; \sigma\right)$}

The constraints on $\sigma$ and the choice of the sets $\left\{B^{(a)}\right\},\left\{C^{(a)}\right\}$ derived in Section 5.3 are necessary but not sufficient for getting a non-vanishing contribution to $\mathrm{N}_{\mathrm{KS}}^{(n+1)}\left(\left\{\alpha_{i}\right\} ; \sigma\right)$. Nevertheless it will be useful at this stage to compare them with the constraints on $\sigma$ needed for $\mathrm{N}_{\text {higgs }}^{(n+1)}\left(\left\{\alpha_{i}\right\} ; \sigma\right)$ to be non-vanishing. According to (2.1) the latter constraints are given by:

$$
\beta_{k}\left\{\begin{array}{ll}
<\bar{\alpha} & \text { for } \sigma(k+1)>\sigma(k), \\
>\bar{\alpha} & \text { for } \sigma(k+1)<\sigma(k),
\end{array} \quad \text { for } 1 \leq k \leq n .\right.
$$

Since $\sigma(R)=n+1$ is larger than both $\sigma(R-1)$ and $\sigma(R+1)$, (5.37) for $k=R-1$ and $k=R$ gives:

$$
\beta_{R-1}<\bar{\alpha}, \quad \beta_{R}>\bar{\alpha}
$$


The conditions on $\beta_{R-1}, \beta_{R}$ given in (5.38) agree with the corresponding constraints (5.27) required for $\mathrm{N}_{\mathrm{KS}}^{(n+1)}$ to be non-vanishing. Furthermore, comparing (5.37) with (5.30), (5.31) we see that in the range $1 \leq k<P$ and $Q \leq k \leq n$, the condition on $\left(\beta_{k}, \widetilde{\alpha}_{k}, \widetilde{\alpha}_{k+1}\right)$ needed for getting non-zero $\mathrm{N}_{\text {higgs }}^{(n+1)}$ agrees with the condition on $\left(\beta_{k}, \widetilde{\alpha}_{k}, \widetilde{\alpha}_{k+1}\right)$ needed for getting nonzero $\mathrm{N}_{\mathrm{KS}}^{(n+1)}$. Thus, we need to focus on the $\left(\beta_{k}, \widetilde{\alpha}_{k}, \widetilde{\alpha}_{k+1}\right)$ 's for $P \leq k \leq Q-1$ and show that for these also the conditions agree. Since all the $\beta_{k}$ 's for $P \leq k \leq R-1$ are on the same side of $\bar{\alpha}$ and all the $\beta_{k}$ 's for $R \leq k<Q$ are on the same side of $\bar{\alpha}$, we see from (5.38) that we have $\beta_{k}<\bar{\alpha}$ for $P \leq k \leq R-1$ and $\beta_{k}>\bar{\alpha}$ for $R \leq k \leq Q-1$. Thus, (5.37) takes a simple form in the range $P \leq k \leq Q-1$ :

$$
\begin{array}{ll}
\sigma(k+1)>\sigma(k) & \text { for } P \leq k \leq R-1 \\
\sigma(k+1)<\sigma(k) & \text { for } R \leq k \leq Q-1
\end{array}
$$

i.e., the $\widetilde{\alpha}_{i}$ 's between $P$ and $R$ must be in the increasing sequence and the $\widetilde{\alpha}_{i}$ 's between $R$ and $Q$ must be in the decreasing sequence. Furthermore for configurations satisfying (5.39), $\mathrm{N}_{\text {higgs }}^{(n+1)}$ given in (2.1) takes the form

$$
s(\sigma)-1=(Q-R)+\sum_{k=1}^{P-1} \Theta(\sigma(k)-\sigma(k+1))+\sum_{k=Q}^{n} \Theta(\sigma(k)-\sigma(k+1)),
$$

where the additive factor of $Q-R$ arises from the contribution for $R \leq k \leq$ $Q-1$. Thus, it remains to prove that for configurations satisfying (5.28), $(5.30),(5.31)$,

(1) $\mathrm{N}_{\mathrm{KS}}^{(n+1)}$ is non-vanishing only for configurations, which satisfy (5.39).

(2) For these configurations $\mathrm{N}_{\mathrm{KS}}^{(n+1)}$ computed from (5.19) agrees with $\mathrm{N}_{\text {higgs }}^{(n+1)}$ given in (5.40).

\subsection{Proof of $\mathrm{N}_{\mathrm{KS}}^{(n+1)}=\mathrm{N}_{\text {higgs }}^{(n+1)}$}

We shall now compute $\mathrm{N}_{\mathrm{KS}}^{(n+1)}$ for a given permutation $\sigma$ and show that the result agrees with that of $\mathrm{N}_{\text {higgs }}^{(n+1)}$. We shall begin by analyzing the constraints on $\widetilde{\alpha}_{k}, \beta_{k}$ for $k \leq R-1$, i.e., for points to the left of $R$. By using the deformation we have set the $\widetilde{\alpha}_{i}$ 's in the range $P<k \leq R-1$ to be almost zero but so far we have not said anything about their relative magnitudes. 
We now note that the order in which the vectors are reduced to almost zero during our manipulation is $\left(\widetilde{\alpha}_{R-1}, \widetilde{\alpha}_{R-2}, \ldots, \widetilde{\alpha}_{P+1}\right)$, i.e., we first make $\widetilde{\alpha}_{R-1}$ almost zero, then $\widetilde{\alpha}_{R-2}$ almost zero and so on. Thus we can arrange the deformations such that the magnitudes of the $\widetilde{\alpha}_{i}$ are arranged in the order:

$$
\left|\widetilde{\alpha}_{R-1}\right| \ll\left|\widetilde{\alpha}_{R-2}\right| \ll \cdots \ll\left|\widetilde{\alpha}_{P+1}\right|,
$$

where now the inequalities refer to standard inequalities between ordinary numbers. This leads to the equation

$$
\sum_{i=\ell}^{\ell^{\prime}} \widetilde{\alpha}_{i} \simeq \widetilde{\alpha}_{\ell} \text { for } P<\ell \leq \ell^{\prime} \leq R-1 .
$$

It now follows that

$$
\delta^{(a)}=\sum_{i=I_{a-1}}^{I_{a}-1} \widetilde{\alpha}_{i} \simeq \widetilde{\alpha}_{I_{a-1}}, \quad \text { for } a \geq 2,
$$

since we have shown earlier that $I_{1}>P$ and hence $I_{a-1}>P$ for $a \geq 2$. The condition (5.13) and the fact that $\delta^{(1)}$ is almost parallel to $\bar{\alpha}$ now gives

$$
\bar{\alpha}>\widetilde{\alpha}_{I_{1}}>\widetilde{\alpha}_{I_{2}}>\cdots>\widetilde{\alpha}_{I_{p-1}}
$$

Let us now examine condition (5.16) by expressing it as

$$
\begin{aligned}
& \delta^{(a)}<\sum_{i=k+1}^{I_{a}-1} \alpha_{\sigma(i)} \quad \text { for } \sigma(k+1)>\sigma(k), \\
& \delta^{(a)}>\sum_{i=k+1}^{I_{a}-1} \alpha_{\sigma(i)} \quad \text { for } \sigma(k+1)<\sigma(k), \\
& I_{a-1} \leq k \leq I_{a}-2 .
\end{aligned}
$$

Using $\widetilde{\alpha}_{i} \equiv \alpha_{\sigma(i)}$ and (5.42), (5.43) we can write this as

$$
\begin{gathered}
\widetilde{\alpha}_{I_{a-1}}<\widetilde{\alpha}_{k+1} \quad \text { for } \quad \widetilde{\alpha}_{k+1}>\widetilde{\alpha}_{k}, \quad \widetilde{\alpha}_{I_{a-1}}>\widetilde{\alpha}_{k+1} \quad \text { for } \quad \widetilde{\alpha}_{k+1}<\widetilde{\alpha}_{k}, \\
\text { for } I_{a-1} \leq k \leq I_{a}-2, \quad a \geq 2, \\
\bar{\alpha}<\widetilde{\alpha}_{k+1} \text { for } \quad \widetilde{\alpha}_{k+1}>\widetilde{\alpha}_{k}, \quad \bar{\alpha}>\widetilde{\alpha}_{k+1} \quad \text { for } \quad \widetilde{\alpha}_{k+1}<\widetilde{\alpha}_{k} \\
\text { for } P \leq k \leq I_{1}-2 .
\end{gathered}
$$

A similar set of constraints can be derived for the $\widetilde{\alpha}_{i}$ 's for $R<i<Q$ by working on the other side of $R$. 
Finally we have to worry about the constraint (5.14). Since we have the $\beta_{i}$ 's for $P \leq i \leq R-1$ and the $\beta_{i}$ 's for $R \leq i<Q$ on opposite sides of $\bar{\alpha}$, it follows from our previous discussion that while manipulating the elements on the left-hand side of $R$, the elements on the right-hand side of $R$ remain fixed. At the end of the first set of deformations $\widetilde{\alpha}_{n+1}$ as well as all the $\widetilde{\alpha}_{i}$ 's on the right-hand side of $R$ remain finite. During the second set of deformations involving elements on the right-hand side of $R$ also $\widetilde{\alpha}_{n+1}$ remains finite almost till the end, and becomes almost zero only at the very last stage. Thus its magnitude can be taken to be larger than that of all the other almost zero $\widetilde{\alpha}_{i}$ 's. We can now consider three possible situations depending on the value we take for $k$ in (5.14):

(1) The $\widehat{\gamma}^{(i)}$ 's which appear in the sum on the left-hand side of (5.14) contains only $\delta^{(a)}$ 's or $\tau^{(a)}$ 's for $a \geq 2$. Since these are smaller in magnitude compared to $\alpha_{n+1}$, while testing (5.14) we can replace the left-hand side of this equation by $\alpha_{n+1}$. In such cases, these equations hold trivially since any linear combinations of the $\alpha_{i}$ 's with non-negative coefficients is $<\alpha_{n+1}$. Let $\ell_{0}$ be the integer such that for all $k<\ell_{0}$ the situation is as described above, i.e., for all $k<\ell_{0}, \widehat{\gamma}^{(k)}$ corresponds to either $\delta^{(a)}$ or $\tau^{(a)}$ with $a \geq 2$.

(2) For $k=\ell_{0}$ the $\widehat{\gamma}^{\left(\ell_{0}\right)}$ in the sum is either $\delta^{(1)}$ or $\tau^{(1)}$ depending on whether $\delta^{(1)}<\tau^{(1)}$ or $\tau^{(1)}<\delta^{(1)}$. Let us for definiteness assume that this is $\tau^{(1)}$. Now the left-hand side of (5.14) will become almost equal to $\tau^{(1)}$ and hence is almost parallel to $\bar{\alpha}$. But now the $\widehat{\gamma}^{\left(\ell_{0}+1\right)}$ on the right hand of the equation is either $\delta^{(a)}$ or $\tau^{(a)}$ for some $a \geq 2$ and hence is, by Equations (5.43), (5.44) and the corresponding equations for $\tau^{(a)},<\bar{\alpha}$ and not almost parallel to $\bar{\alpha}$. Thus, (5.14) still holds. The same argument holds for all subsequent values of $k$ till $k=p+q-2$, with the left-hand side almost parallel to $\bar{\alpha}$ and the right-hand side $<\bar{\alpha}$ and not almost parallel to $\bar{\alpha}$.

(3) For $k=p+q-1$ the right-hand side of (5.14) becomes $\widehat{\gamma}^{(p+q)}=\delta^{(1)}$. The left-hand side of the equation is now $\bar{\alpha}-\delta^{(1)}$. Since both sides are almost parallel to $\bar{\alpha}$ we need to carry out the comparison with a little more care. For this note that (5.14), which requires $\bar{\alpha}-\delta^{(1)}>\delta^{(1)}$ is equivalent to requiring $\delta^{(1)}<\bar{\alpha}$. Since $\delta^{(1)}=\beta_{I_{1}-1}$ and $P \leq I_{1}-1 \leq$ $R-1,(5.28)$ ensures that $\delta^{(1)}<\bar{\alpha}$. Thus (5.14) still holds.

From this we conclude that (5.14) does not impose any additional constraints on the $\beta_{k}$ 's and $\widetilde{\alpha}_{k}$ 's once the other conditions have been satisfied. A similar conclusion holds for the $\beta_{k}$ 's and $\widetilde{\alpha}_{k}$ 's for $k>R$. 
We now need to compute the contribution to $\mathrm{N}_{\mathrm{KS}}^{(n+1)}$ from the allowed configurations. The net contribution to $\mathrm{N}_{\mathrm{KS}}^{(n+1)}$ comes from summing the weight factor given in (5.19) over all possible choice of $p$ and $q$ and the integers $I_{1}, \ldots, I_{p-1}, J_{1}, \ldots, J_{q-1}$ subject to all the constraints. Now of the constraints given in (5.13) to (5.17), the constraints (5.14) (or equivalently (5.15)) are the only ones which involve both the sets $\left\{B_{a}\right\}$ and $\left\{C_{a}\right\}$. Since we have argued that these constraints are automatically satisfied when the other constraints are satisfied, the constraints on $\left\{p, I_{1}, \ldots, I_{p-1}\right\}$ and $\left\{q, J_{1}, \ldots, J_{q-1}\right\}$ become independent of each other and hence we can carry out the sum over $\left\{p, I_{1}, \ldots, I_{p-1}\right\}$ and $\left\{q, J_{1}, \ldots, J_{q-1}\right\}$ independently, and express (5.19) as

$$
\begin{aligned}
& \sum_{\substack{\text { allowed values of } \\
\left\{p, I_{1}, \ldots . I_{p-1}\right\}}}(-1)^{\sum_{a=1}^{p} \sum_{k=I_{a-1}}^{I_{a}-1}} \Theta(\sigma(k)-\sigma(k+1)) \\
& \times \sum_{\substack{\text { allowed values of } \\
\left\{q, J_{1}, \ldots, J_{q-1}\right\}}}(-1)^{q+\sum_{a=1}^{q} \sum_{\substack{k=J_{a-1} \\
J_{a}-1}} \Theta(\sigma(k)-\sigma(k+1))} .
\end{aligned}
$$

We shall first carry out the sum over $p$ and $I_{1}, \ldots, I_{p-1}$. Besides the constraints given in $(5.44),(5.46)$, we also need to account for the constraint (5.29) that $I_{1}$ must be $\geq P+1$. Taking into account all the constraints and introducing a new variable $k=p-1$, we may express the net contribution as:

$$
\begin{aligned}
& (-1)^{\sum_{i=1}^{P-1} \Theta\left(\widetilde{\alpha}_{i}, \widetilde{\alpha}_{i+1}\right)}\left\{\prod_{i=P}^{R-2}\left[\Theta\left(\widetilde{\alpha}_{i+1}, \bar{\alpha}\right) \Theta\left(\widetilde{\alpha}_{i+1}, \widetilde{\alpha}_{i}\right)\right)-\Theta\left(\bar{\alpha}, \widetilde{\alpha}_{i+1}\right) \Theta\left(\widetilde{\alpha}_{i}, \widetilde{\alpha}_{i+1}\right)\right] \\
& \quad+\sum_{k=1}^{R-P-1} \sum_{I_{1}=P+1}^{R-1} \sum_{I_{2}=I_{1}+1}^{R-1} \sum_{I_{3}=I_{2}+1}^{R-1} \ldots \sum_{I_{k}=I_{k-1}+1}^{R-1}\left\{\Theta\left(\bar{\alpha}, \alpha_{I_{1}}\right) \prod_{\ell=1}^{k-1} \Theta\left(\widetilde{\alpha}_{I_{\ell}}, \widetilde{\alpha}_{I_{\ell+1}}\right)\right. \\
& \quad \times \prod_{i=P}^{I_{1}-2}\left[\Theta\left(\widetilde{\alpha}_{i+1}, \bar{\alpha}\right) \Theta\left(\widetilde{\alpha}_{i+1}, \widetilde{\alpha}_{i}\right)-\Theta\left(\bar{\alpha}, \widetilde{\alpha}_{i+1}\right) \Theta\left(\widetilde{\alpha}_{i}, \widetilde{\alpha}_{i+1}\right)\right] \\
& \left.\quad \times \prod_{\ell=1}^{k-1} \prod_{i=I_{\ell}}^{I_{\ell+1}-2}\left[\Theta\left(\widetilde{\alpha}_{i+1}, \widetilde{\alpha}_{I_{\ell}}\right) \Theta\left(\widetilde{\alpha}_{i+1}, \widetilde{\alpha}_{i}\right)\right)-\Theta\left(\widetilde{\alpha}_{I_{\ell}}, \widetilde{\alpha}_{i+1}\right) \Theta\left(\widetilde{\alpha}_{i}, \widetilde{\alpha}_{i+1}\right)\right] \\
& \left.\left.\left.\quad \times \prod_{i=I_{k}}^{R-2}\left[\Theta\left(\widetilde{\alpha}_{i+1}, \widetilde{\alpha}_{I_{k}}\right) \Theta\left(\widetilde{\alpha}_{i+1}, \widetilde{\alpha}_{i}\right)\right)-\Theta\left(\widetilde{\alpha}_{I_{k}}, \widetilde{\alpha}_{i+1}\right) \Theta\left(\widetilde{\alpha}_{i}, \widetilde{\alpha}_{i+1}\right)\right]\right\}\right\} .
\end{aligned}
$$


In this expression , $k=p-1$ denotes the total number of $B_{a}$ 's other than $B_{1}$. The first term represents the $k=0$ term where there is a single set $B_{1}$ containing all the elements from 1 to $R-1$, and the product of the $\Theta$ 's account for the constraint (5.46). In the other terms, the $I_{\ell}$ 's mark the beginning of the set $B_{\ell+1}$ as in (5.8). The product of the $\Theta$ 's in the second line of (5.48) impose the constraints (5.44) and the $\Theta$ 's in the last three lines of (5.48) impose the constraints (5.46). The $-\operatorname{sign}$ between the two terms in the first line and the last three lines originate from the $(-1)^{\sum_{a=1}^{p} \sum_{i=I_{a-1}}^{I_{a}-1} \Theta(\sigma(i)-\sigma(i+1))}$ factor in (5.47). It takes care of the contribution from the pairs $(i$, $i+1$ ) for $P \leq i \leq R-1$, but not for values of $i$ outside this range (e.g., for $1 \leq i<P$ ) which has been included as an overall factor at the beginning of $(5.48)$.

Using the fact that $\Theta\left(\widetilde{\alpha}_{i}, \widetilde{\alpha}_{j}\right)=\Theta(\sigma(i)-\sigma(j))$ where the second $\Theta$ denotes an ordinary step function, we can convert (5.48) into a purely combinatoric expression as follows. Let $k_{0}$ be the integer for which $\alpha_{k_{0}}<\bar{\alpha}<$ $\alpha_{k_{0}+1}$. We now define $c$ to be any number between $k_{0}$ and $k_{0}+1$. Then $\Theta\left(\widetilde{\alpha}_{i}, \bar{\alpha}\right)=\Theta(\sigma(i)-c)$ and we may rewrite (5.48) as

$$
\begin{aligned}
& (-1)^{\sum_{i=1}^{P-1} \Theta(\sigma(i)-\sigma(i+1))}\left[\prod_{i=P}^{R-2}[\Theta(\sigma(i+1)-c) \Theta(\sigma(i+1)-\sigma(i))\right. \\
& -\Theta(c-\sigma(i+1)) \Theta(\sigma(i)-\sigma(i+1))]+\sum_{k=1}^{R-P-1} \sum_{I_{1}=P+1}^{R-1} \sum_{I_{2}=I_{1}+1}^{R-1} \\
& \times \sum_{I_{3}=I_{2}+1}^{R-1} \sum_{I_{k}=I_{k-1}+1}^{R-1}\left\{\Theta\left(c-\sigma\left(I_{1}\right)\right) \prod_{\ell=1}^{k-1} \Theta\left(\sigma\left(I_{\ell}\right)-\sigma\left(I_{\ell+1}\right)\right)\right. \\
& \times \prod_{i=P}^{I_{1}-2}[\Theta(\sigma(i+1)-c) \Theta(\sigma(i+1)-\sigma(i))-\Theta(c-\sigma(i+1)) \\
& \times \Theta(\sigma(i)-\sigma(i+1))] \prod_{\ell=1}^{k-1} \prod_{i=I_{\ell}}^{I_{\ell+1}-2}\left[\Theta\left(\sigma(i+1)-\sigma\left(I_{\ell}\right)\right)\right. \\
& \left.\times \Theta(\sigma(i+1)-\sigma(i))-\Theta\left(\sigma\left(I_{\ell}\right)-\sigma(i+1)\right) \Theta(\sigma(i)-\sigma(i+1))\right] \\
& \times \prod_{i=I_{k}}^{R-2}\left[\Theta\left(\sigma(i+1)-\sigma\left(I_{k}\right)\right) \Theta(\sigma(i+1)-\sigma(i))\right. \\
& \left.\left.-\Theta\left(\sigma\left(I_{k}\right)-\sigma(i+1)\right) \Theta(\sigma(i)-\sigma(i+1))\right]\right\}
\end{aligned}
$$


We now make use of the identity - proved in Appendix A - that for any function $f(i)$ satisfying $f(i) \neq f(j)$ for $i \neq j$ and any constant $c$, we have

$$
\begin{aligned}
& \prod_{i=1}^{N-1}[\Theta(f(i+1)-c) \Theta(f(i+1)-f(i))-\Theta(c-f(i+1)) \Theta(f(i)-f(i+1))] \\
& +\sum_{k=1}^{N-1} \sum_{I_{1}=2}^{N} \sum_{I_{2}=I_{1}+1}^{N} \sum_{I_{3}=I_{2}+1}^{N} \ldots \sum_{I_{k}=I_{k-1}+1}^{N} \\
& \times\left\{\Theta\left(c-f\left(I_{1}\right)\right) \prod_{\ell=1}^{k-1} \Theta\left(f\left(I_{\ell}\right)-f\left(I_{\ell+1}\right)\right) \prod_{i=1}^{I_{1}-2}[\Theta(f(i+1)-c)\right. \\
& \times \Theta(f(i+1)-f(i))-\Theta(c-f(i+1)) \Theta(f(i)-f(i+1))] \\
& \times \prod_{\ell=1}^{k-1} \prod_{i=I_{\ell}}^{I_{\ell+1}-2}\left[\Theta\left(f(i+1)-f\left(I_{\ell}\right)\right) \Theta(f(i+1)-f(i))\right. \\
& \left.-\Theta\left(f\left(I_{\ell}\right)-f(i+1)\right) \Theta(f(i)-f(i+1))\right] \prod_{i=I_{k}}^{N-1}\left[\Theta\left(f(i+1)-f\left(I_{k}\right)\right)\right. \\
& \left.\left.\times \Theta(f(i+1)-f(i))-\Theta\left(f\left(I_{k}\right)-f(i+1)\right) \Theta(f(i)-f(i+1))\right]\right\} \\
& \quad=\prod_{i=1}^{N-1} \Theta(f(i+1)-f(i)) .
\end{aligned}
$$

It is easy to see that except for the factor in the first line of (5.49), the lefthand side of (5.50) reduces to (5.49) under the identification $N=R-P$, $f(i)=\sigma(i+P-1)$ and a renaming of the variables $I_{\ell}$ to $I_{\ell}-P+1$. Thus, (5.49) becomes

$$
(-1)^{\sum_{i=1}^{P-1} \Theta(\sigma(i)-\sigma(i+1))} \prod_{i=P}^{R-2} \Theta(\sigma(i+1)-\sigma(i)) .
$$

The product of the $\Theta$ 's coincide with the first set of conditions given in (5.39).

The case where all the $\widetilde{\alpha}_{i}$ 's for $1 \leq i \leq R-1$ are almost zero requires special treatment. In this case, (5.41) holds all the way to $\widetilde{\alpha}_{1}$ with $\left|\widetilde{\alpha}_{1}\right|$ being the largest and (5.42) holds for $1 \leq \ell \leq \ell^{\prime} \leq R-1$. Thus we have $\delta^{(1)} \simeq \widetilde{\alpha}_{1}$ 
and the analog of (5.49) takes the form

$$
\begin{aligned}
& \prod_{i=1}^{R-2}[\Theta(\sigma(i+1)-\sigma(1)) \Theta(\sigma(i+1)-\sigma(i))-\Theta(c-\sigma(i+1)) \\
& \quad \times \Theta(\sigma(i)-\sigma(i+1))]+\sum_{k=1}^{R-2} \sum_{I_{1}=2}^{R-1} \sum_{I_{2}=I_{1}+1}^{R-1} \sum_{I_{3}=I_{2}+1}^{R-1} \ldots \sum_{I_{k}=I_{k-1}+1}^{R-1} \\
& \quad \times\left\{\Theta\left(\sigma(1)-\sigma\left(I_{1}\right)\right) \prod_{\ell=1}^{k-1} \Theta\left(\sigma\left(I_{\ell}\right)-\sigma\left(I_{\ell+1}\right)\right) \prod_{i=1}^{I_{1}-2}[\Theta(\sigma(i+1)-\sigma(1))\right. \\
& \quad \times \Theta(\sigma(i+1)-\sigma(i))-\Theta(\sigma(1)-\sigma(i+1)) \Theta(\sigma(i)-\sigma(i+1))] \\
& \quad \times \prod_{\ell=1}^{k-1} \prod_{i=I_{\ell}}^{I_{\ell+1}-2}\left[\Theta\left(\sigma(i+1)-\sigma\left(I_{\ell}\right)\right) \Theta(\sigma(i+1)-\sigma(i))-\Theta\left(\sigma\left(I_{\ell}\right)-\sigma(i+1)\right)\right. \\
& \quad \times \Theta(\sigma(i)-\sigma(i+1))] \prod_{i=I_{k}}^{R-2}\left[\Theta\left(\sigma(i+1)-\sigma\left(I_{\ell}\right)\right) \Theta(\sigma(i+1)-\sigma(i))\right. \\
& \left.\left.\quad-\Theta\left(\sigma\left(I_{\ell}\right)-\sigma(i+1)\right) \Theta(\sigma(i)-\sigma(i+1))\right]\right\} .
\end{aligned}
$$

This is identical to the left-hand side of (5.50) with $c$ replaced by $\sigma(1), f(i)$ replaced by $\sigma(i)$ and $N$ replaced by $R-1$. Thus, the result is

$$
\prod_{i=1}^{R-2} \Theta(\sigma(i+1)-\sigma(i)) .
$$

We can analyze the contribution from the right-hand side of $R$ by summing over all possible choices of $q$ and $J_{1}, \ldots, J_{q-1}$. Since this is related to the previous analysis by a reversal of permutation the result can be read out from our previous analysis. However there are two important effects which need to be taken into account. First of all (5.47) has a factor of $(-1)^{q}$ which in the present context will turn into $(-1)^{k+1}$. Second in order to convert this problem to the previous case we need to flip the sign of each term inside [ ] in (5.48) since the pair $(i, i+1)$ goes over to $\left(i^{\prime}+1, i^{\prime}\right)$ for some $i^{\prime}$ under permutation reversal. This gives a factor of $(-1)^{f}$ where $f$ essentially counts the total number of nearest-neighbor pairs between $R+1$ and $Q$ except the links, which connect the end point of $C_{a}$ to the starting point of $C_{a-1}$ for $2 \leq a \leq q$. Thus, we have $f=Q-R-1-(q-1)=Q-R-k-1$ and 
hence the net factor is

$$
(-1)^{f+k+1}=(-1)^{Q-R}
$$

The result of summing over the locations of $J_{1}, \ldots, J_{q-1}$ in the range $R$ to $Q$ is now given by

$$
(-1)^{\sum_{i=Q}^{n} \Theta(\sigma(i)-\sigma(i+1))}(-1)^{Q-R} \prod_{j=R+1}^{Q-1} \Theta(\sigma(j)-\sigma(j+1))
$$

both when $Q<n+1$ and when $Q=n+1$. This shows that between $R$ and $Q$ the $\sigma(j)$ 's must form a decreasing sequence. The first factor in (5.55) is the contribution from the points between $Q$ and $n+1$.

Equations (5.51) and (5.55) and the fact that $\sigma(R)=n+1>$ $\sigma(R-1), \sigma(R+1)$ together give

$\sigma(k+1)>\sigma(k)$ for $P \leq k \leq R-1, \quad \sigma(k+1)<\sigma(k)$ for $R \leq k \leq Q-1$.

which precisely correspond to the condition (5.39) for $g_{\text {higgs }}$ to be nonvanishing. The net contribution to $\mathrm{N}_{\mathrm{KS}}^{(n+1)}$ for a configuration satisfying (5.56) and the other conditions described in Section 5.3 is now given by the product of (5.51) and (5.55) (with $P$ replaced by 1 or $Q$ replaced by $n+1$ in special cases). The result is

$$
\begin{gathered}
\mathrm{N}_{\mathrm{KS}}^{(n+1)}\left(\left\{\alpha_{i}\right\} ; \sigma\right)=(-1)^{r(\sigma)} \\
r(\sigma)=Q-R+\sum_{i=1}^{P-1} \Theta(\sigma(i)-\sigma(i+1))+\sum_{i=Q}^{n} \Theta(\sigma(i)-\sigma(i+1)) .
\end{gathered}
$$

This is in perfect agreement with (5.40).

Finally, we note that the very special cases when $R$ itself lies at either end can also be easily incorporated in this analysis. For example if $R=1$ we simply need to drop the $\sum_{i=1}^{P-1} \Theta(\sigma(i)-\sigma(i+1))$ term and if $R=n+1$ we shall need to drop the $Q-R+\sum_{i=Q}^{n} \Theta(\sigma(i)-\sigma(i+1))$ term. These are also in agreement with the corresponding formula (5.40) for $\mathrm{N}_{\text {higgs }}^{(n+1)}$. 


\section{An alternative approach to proving the equivalence of the Higgs branch and KS wall-crossing formulæ}

In Sections 4 and 5, we gave a proof of the equality of $g_{\mathrm{KS}}$ and $g_{\mathrm{higgs}}$ for generic choice of the arguments $\alpha_{1}, \ldots, \alpha_{n}$. This proof used the definition of $g_{\mathrm{KS}}$ given in Section 3 based on the universal enveloping algebra of the Lie algebra (3.1). The referee suggested a simpler approach based on the quantum torus algebra

$$
e_{\gamma} e_{\gamma^{\prime}}=\left(y-y^{-1}\right)^{-1}(-y)^{\left\langle\gamma, \gamma^{\prime}\right\rangle} e_{\gamma+\gamma^{\prime}}
$$

which provides a representation of (3.1). The KS wall-crossing formula takes the form:

$$
\prod_{\gamma \text { clockwise }} \exp \left[\bar{\Omega}_{\text {ref }}^{-}(\gamma, y) e_{\gamma}\right]=\prod_{\gamma \text { anti-clockwise }} \exp \left[\bar{\Omega}_{\mathrm{ref}}^{+}(\gamma, y) e_{\gamma}\right]
$$

where $\prod_{\gamma \text { clockwise }}\left(\prod_{\gamma \text { anti-clockwise }}\right)$ denotes product over all vectors $\gamma \in \Lambda$, and the terms in the product arranged such that as we move from the left to the right the corresponding $\gamma$ 's are arranged clockwise (anti-clockwise) in the 2D plane. Expanding both sides using (6.1), and collecting the coefficient of $e_{\gamma}$ from each side, we get

$$
\begin{aligned}
& \sum_{m=1}^{\infty} \sum_{\substack{\beta_{1}+\cdots \beta_{m}=\gamma, \beta_{1} \leq \beta_{2} \leq \cdots \leq \beta_{m} \\
\beta_{m} \in \beta^{\prime}}} \frac{1}{\left|\operatorname{Aut}\left(\left\{\beta_{1}, \ldots \beta_{m}\right\}\right)\right|}\left(y-y^{-1}\right)^{-m}(-y)^{\sum_{i<j} \beta_{i j}} \\
& \times \prod_{i=1}^{m} \bar{\Omega}_{\mathrm{ref}}^{-}\left(\beta_{i}, y\right) \\
& =\sum_{n=1}^{\infty} \sum_{\substack{\alpha_{1}+\cdots \alpha_{n}=\gamma, \alpha_{1} \leq \alpha_{2} \leq \cdots \leq \alpha_{n} \\
\alpha_{1}, \alpha_{n}}} \frac{1}{\left|\operatorname{Aut}\left(\left\{\alpha_{1}, \ldots \alpha_{n}\right\}\right)\right|} \\
& \quad \times\left(y-y^{-1}\right)^{-n}(-y)^{-\sum_{i<j} \alpha_{i j}} \prod_{i=1}^{n} \bar{\Omega}_{\mathrm{ref}}^{+}\left(\alpha_{i}, y\right)
\end{aligned}
$$

where by $\beta_{i}=\beta_{j}$ we mean $\beta_{i}$ is either equal or parallel to $\beta_{j}$. We now substitute in the left-hand side of this equation the expression for $\bar{\Omega}_{\text {ref }}^{-}\left(\beta_{i}, y\right)$ in terms of $\bar{\Omega}_{\text {ref }}^{+}\left(\alpha_{i}, y\right)$ 's using Equations (1.3) with $g_{\text {ref }}$ replaced by $g_{\mathrm{KS}}$. This 
gives

$$
\begin{aligned}
& \sum_{m=1}^{\infty} \sum_{\substack{\beta_{1}, \ldots \beta_{m} \in \Lambda \\
\beta_{1}+\cdots \beta_{m}=\gamma, \beta_{1} \leq \beta_{2} \leq \cdots \leq \beta_{m}}} \frac{1}{\left|\operatorname{Aut}\left(\left\{\beta_{1}, \ldots \beta_{m}\right\}\right)\right|}\left(y-y^{-1}\right)^{-m}(-y)^{\sum_{i<j} \beta_{i j}} \\
& \times \prod_{k=1}^{m} \sum_{s_{k} \geq 1} \sum_{\text {unordered }} \sum_{\operatorname{set} \alpha_{1}^{(k)}, \ldots, \alpha_{s_{k}}^{(k)} \in \Lambda} \frac{g_{\mathrm{KS}}\left(\alpha_{1}^{(k)}, \ldots, \alpha_{s_{k}}^{(k)}\right)}{\left|\operatorname{Aut}\left(\left\{\alpha_{1}^{(k)}, \ldots, \alpha_{s_{k}}^{(k)}\right\}\right)\right|} \\
& \alpha_{1}^{(k)}+\cdots+\alpha_{s_{k}}^{(k)}=\beta_{k} \\
& \times \prod_{i=1}^{s_{k}} \bar{\Omega}_{\mathrm{ref}}^{+}\left(\alpha_{i}^{(k)}, y\right) \\
& =\sum_{n=1}^{\infty} \sum_{\substack{\alpha_{1}, \ldots \alpha_{n} \in \Lambda \\
\alpha_{1}+\cdots \alpha_{n}=\gamma, \alpha_{1} \leq \alpha_{2} \leq \cdots \leq \alpha_{n}}} \frac{1}{\left|\operatorname{Aut}\left(\left\{\alpha_{1}, \ldots \alpha_{n}\right\}\right)\right|} \\
& \times\left(y-y^{-1}\right)^{-n}(-y)^{-\sum_{i<j} \alpha_{i j}} \prod_{i=1}^{n} \bar{\Omega}_{\mathrm{ref}}^{+}\left(\alpha_{i}, y\right) \text {. }
\end{aligned}
$$

Comparing the coefficient of $\prod_{i=1}^{n} \bar{\Omega}_{\text {ref }}^{+}\left(\alpha_{i}, y\right)$ in two sides of this equation we can get a set of recursion relations involving $g_{\mathrm{KS}}\left(\alpha_{1}, \ldots, \alpha_{n}\right)$ from which we can determine $g_{\mathrm{KS}}\left(\alpha_{1}, \ldots, \alpha_{n}\right)$. Thus proving the equivalence of $g_{\mathrm{KS}}$ and $g_{\text {higgs }}$ is equivalent to checking if $g_{\text {higgs }}$ satisfies the same set of relations (6.4) as $g_{\mathrm{KS}}$. For this we replace $g_{\mathrm{KS}}$ in (6.4) by the expression for $g_{\text {higgs }}$ given in (2.1) for generic arguments and try to verify the resulting equation. Comparing the coefficients of $\prod_{i=1}^{n} \bar{\Omega}_{\text {ref }}^{+}\left(\alpha_{i}, y\right)$ on both sides of this equation for generic $\alpha_{i}$ 's for which

$$
\alpha_{1}<\alpha_{2}<\cdots<\alpha_{n}
$$

and $\sum_{i \in A} \alpha_{i}$ and $\sum_{i \in B} \alpha_{i}$ are different from each other for any choice of non-overlapping sets $A$ and $B$ of $\{1,2, \ldots, n\}$, we get:

$$
\begin{aligned}
& \sum_{\substack{\text { permutations } \sigma \\
\text { of } 1,2, \ldots n}} \sum_{m=1}^{n} \sum_{\substack{n_{1}, \ldots, n_{m} \\
0 \equiv n_{0}<n_{1}<n_{2}<\cdots<n_{m} \equiv n}}\left(y-y^{-1}\right)^{-m} \\
& \times\left\{\prod_{k=1}^{m-1} \Theta\left(\beta_{k+1}, \beta_{k}\right)\right\}(-y)^{\sum_{k=1}^{m-1} \sum_{l=k+1}^{m}\left\langle\beta_{k}, \beta_{l}\right\rangle}(-1)^{n-m}\left(y-y^{-1}\right)^{m-n} \\
& \times(-y)^{\sum_{k=1}^{m} \sum_{i=n_{k-1}+1}^{n_{k}-1} \sum_{j=i+1}^{n_{k}} \alpha_{\sigma(i) \sigma(j)}}(-1)^{\sum_{k=1}^{m} \sum_{i=n_{k-1}+1}^{n_{k}-1} \Theta\left(\alpha_{\sigma(i)}, \alpha_{\sigma(i+1)}\right)}
\end{aligned}
$$




$$
\begin{aligned}
& \left.\times \prod_{k=1}^{m}\left\{\prod_{\substack{i=n_{k-1}+1 \\
\sigma(i)>\sigma(i+1)}}^{n_{k}-1} \Theta\left(\sum_{j=n_{k-1}+1}^{i} \alpha_{\sigma(j)}, \beta_{k}\right) \prod_{\substack{i=n_{k}-1+1 \\
\sigma(i)<\sigma(i+1) \\
n_{k}}}^{n_{k}-1} \alpha_{k}, \sum_{j=n_{k-1}+1}^{i} \alpha_{\sigma(j)}\right)\right\} \\
& =\left(y-y^{-1}\right)^{-n}(-y)^{-\sum_{i<j} \alpha_{i j}}, \beta_{k} \equiv \sum_{j=n_{k-1}+1}^{i} \alpha_{\sigma(j)} .
\end{aligned}
$$

The sum over $\sigma$ runs over all permutations of $\{1,2, \ldots, n\}$. For a fixed $\sigma$, the different choices of the integers $n_{k}$ correspond to different partitioning of the ordered set $\left\{\alpha_{\sigma(1)}, \ldots, \alpha_{\sigma(n)}\right\}$. The sum of the vectors inside the partitions from left to right are given by $\beta_{1}, \ldots, \beta_{m}$, satisfying the constraint $\beta_{1}<\beta_{2}<\cdots<\beta_{m}$ as in the left-hand side of (6.4). ${ }^{6}$ After canceling the $\left(y-y^{-1}\right)^{-n}$ factors from the two sides, we note that the net power of $(-y)$ on the left-hand side is given by $\sum_{i=1}^{n-1} \sum_{j=i+1}^{n} \alpha_{\sigma(i) \sigma(j)}$, i.e., the power of $(-y)$ is determined only by the permutation $\sigma$ and is independent of the choice of the integers $m$ and $n_{1}, \ldots, n_{m}$ which partitions the vectors into $\left\{\beta_{1}, \ldots \beta_{m}\right\}$. Comparing the different powers of $y$ on the two sides of (6.6) we now get

$$
\begin{aligned}
& \sum_{m=1}^{n} \sum_{\substack{n_{1}, \ldots, n_{m} \\
0 \equiv n_{0}<n_{1}<n_{2}<\cdots<n_{m} \equiv n}}\left\{\prod_{k=1}^{m-1} \Theta\left(\beta_{k+1}, \beta_{k}\right)\right\} \\
& \times(-1)^{n-m+\sum_{k=1}^{m} \sum_{\substack{\sum_{k}-1 \\
n_{k-1}+1}} \Theta\left(\alpha_{\sigma(i)}, \alpha_{\sigma(i+1)}\right)} \\
& \times \prod_{k=1}^{m}\left\{\prod_{\substack{i=n_{k}-1+1 \\
\sigma(i)>\sigma(i+1)}}^{n_{k}-1} \Theta\left(\sum_{j=n_{k-1}+1}^{i} \alpha_{\sigma(j)}, \beta_{k}\right) \prod_{\substack{i=n_{k-1}+1 \\
\sigma(i)<\sigma(i+1)}}^{n_{k}-1} \Theta\left(\beta_{k}, \sum_{j=n_{k-1}+1}^{i} \alpha_{\sigma(j)}\right)\right\} \\
& \quad= \begin{cases}1 & \text { for } \sigma(1,2, \ldots, n)=(n, n-1, \ldots, 1) \\
0 & \text { otherwise }\end{cases}
\end{aligned}
$$

For a fixed $\sigma$, we shall refer to the choice of $m$ and $\left\{n_{1}, \ldots, n_{m}\right\}$ for which the summand is non-vanishing as an allowed partition.

Our goal now is to prove (6.7). We begin with the case $\sigma(1,2, \ldots, n)=$ $(n, n-1, \ldots, 1)$. In this case the only way to avoid a vanishing contribution from the $\prod_{k=1}^{m-1} \Theta\left(\beta_{k+1}, \beta_{k}\right)$ factor is to choose $m=1, n_{1}=n$. In this case

\footnotetext{
${ }^{6}$ Note that since we are taking the $\alpha_{i}$ 's to be generic, we do not consider the case where some of the $\beta_{k}$ 's are equal or parallel to each other.
} 
the condition (6.5) tells us that

$$
\alpha_{\sigma(i)}>\alpha_{\sigma(i+1)}, \quad \sum_{j=1}^{i} \alpha_{\sigma(j)}>\beta_{1}=\alpha_{1}+\cdots+\alpha_{n} .
$$

Hence, the product of the step functions given in the last line on the left-hand side of (6.7) is 1 since for all $i$ we have $\sigma(i)>\sigma(i+1)$ and $\sum_{j=1}^{i} \alpha_{\sigma(j)}>\beta_{1}$. The sign of the term is $(-1)^{n-1+n-1}=1$. Thus, the result is 1 in agreement with the right-hand side of (6.7).

To deal with the case of other permutations, we note first of all that the right-hand side of (6.7) is independent of the $\alpha_{i}$ 's. Thus we need to show that the left-hand side of this equation must also be invariant under deformations of the $\alpha_{i}$ 's as long as we preserve the order (6.5). This is not manifest, e.g., during such deformations of the $\alpha_{i}$ 's, in a given term in (6.7) $\beta_{k}$ and $\beta_{k+1}$ defined in (6.6) may go from $\beta_{k}<\beta_{k+1}$ to $\beta_{k}>\beta_{k+1}$ and as a result $\Theta\left(\beta_{k+1}, \beta_{k}\right)$ may jump from 1 to 0 . Thus if we choose $n_{1}, \ldots, n_{m}$ such that initially we have $\beta_{k}<\beta_{k+1}$, and the restrictions imposed by the various other step functions in (6.7) are satisfied so that we have a non-zero contribution, during the deformation we may arrive at $\beta_{k}>\beta_{k+1}$ so that this term ceases to contribute. Thus to show that the left-hand side of (6.7) is unchanged during such a deformation we must identify another contribution that either ceases to exist or begins to exist when $\beta_{k}$ and $\beta_{k+1}$ switches order, compensating for the change caused due to the previous effect. We consider two cases separately: $\alpha_{\sigma\left(n_{k}\right)}<\alpha_{\sigma\left(n_{k}+1\right)}$ and $\alpha_{\sigma\left(n_{k}\right)}>\alpha_{\sigma\left(n_{k}+1\right)}$. In the first case it is easy to see that as long as $\beta_{k}<\beta_{k+1}$, the configuration with $m \rightarrow$ $m-1$, and the $\beta_{k}$ 's chosen as $\left\{\beta_{1}, \ldots, \beta_{k-1}, \beta_{k}+\beta_{k+1}, \beta_{k+2}, \ldots, \beta_{m}\right\}$ also contributes to the left-hand side of (6.6), and furthermore, this configuration also ceases to contribute when we cross over to the side where $\beta_{k}>\beta_{k+1}$. Furthermore for $\beta_{k}<\beta_{k+1}$ the contribution from these two configurations have opposite signs so that the sum of the two terms vanishes and there is no net change in the left-hand side of (6.7) as we pass from $\beta_{k}<\beta_{k+1}$ to $\beta_{k}>$ $\beta_{k+1}$. On the other hand if $\alpha_{\sigma\left(n_{k}\right)}>\alpha_{\sigma\left(n_{k}+1\right)}$, then the second configuration does not contribute for $\beta_{k}<\beta_{k+1}$ but does contribute when $\beta_{k}>\beta_{k+1}$ and the sign of the second contribution for $\beta_{k}>\beta_{k+1}$ is the same as that of the first configuration for $\beta_{k}<\beta_{k+1}$. Thus again the net contribution remains unchanged during this process.

The other possible source of jump in the left-hand side of (6.7) is when $\sum_{j=n_{k-1}+1}^{i} \alpha_{\sigma(j)}$ crosses $\beta_{k}$ for some $(i, k)$ during the deformation. In this case we analyze the contribution from the original partition together with 
that of another partition corresponding to $m \rightarrow m+1$, with the $\beta_{i}$ 's given by

$$
\left\{\beta_{1}, \ldots \beta_{k-1}, \sum_{j=n_{k-1}+1}^{i} \alpha_{\sigma(j)}, \sum_{j=i+1}^{n_{k}} \alpha_{\sigma(j)}, \beta_{k+1}, \ldots, \beta_{m}\right\} .
$$

The analysis of the net jump of the left-hand side of (6.7) from these two terms is the same as the one given in the last paragraph, with the roles of the first and the second terms getting exchanged.

This shows that the left-hand side of (6.7) is unchanged under continuous deformation of the $\alpha_{i}$ 's preserving (6.5). Armed with this result we shall now try to prove (6.7) using the method of induction, i.e., we shall assume that the result is valid for $(n-1) \alpha_{i}$ 's and then try to prove it for $n \alpha_{i}$ 's. Using the invariance of the left-hand side of (6.7) under deformations of the $\alpha_{i}$ 's preserving $(6.5)$, we shall choose $\alpha_{\sigma(n)}$ to be small in magnitude compared to all the other $\alpha_{\sigma(i)}$ 's. In this case all the step functions in (6.7), except for the ones which contain $\alpha_{\sigma(n)}$ as one of its arguments, reduce to those for the case of $(n-1)$ vectors $\alpha_{\sigma(1)}, \ldots, \alpha_{\sigma(n-1)}$. Thus the possible allowed partitions are of the form:

$$
\left\{\beta_{1}, \ldots, \beta_{m}+\alpha_{\sigma(n)}\right\}, \quad\left\{\beta_{1}, \ldots, \beta_{m}, \alpha_{\sigma(n)}\right\},
$$

where $\left\{\beta_{1}, \ldots, \beta_{m}\right\}$ is an allowed partition for $(n-1)$ vectors $\alpha_{\sigma(1)}, \ldots$, $\alpha_{\sigma(n-1)}$. We now consider two possibilities:

(1) $\alpha_{\sigma(n)}>\alpha_{\sigma(n-1)}$ : In this case, by examining the additional step functions which involve $\alpha_{\sigma(n)}$ as one of the arguments we see that both the partitions given in (6.9) can contribute only when $\alpha_{\sigma(n)}>\beta_{m}$, and they contribute with opposite sign. Thus we conclude that the net contribution vanishes.

(2) $\alpha_{\sigma(n)}<\alpha_{\sigma(n-1)}$ : In this case the first partition contributes when $\alpha_{\sigma(n)}<$ $\beta_{m}$ and the second partition contributes when $\alpha_{\sigma(n)}>\beta_{m}$. Both contributions are equal, and so we get a non-vanishing contribution that is independent of whether $\alpha_{\sigma(n)}<\beta_{m}$ of $\alpha_{\sigma(n)}>\beta_{m}$. This allows us to sum over all partitions $\left\{\beta_{1}, \ldots, \beta_{m}\right\}$ of $\alpha_{\sigma(1)}, \ldots, \alpha_{\sigma(n-1)}$ freely, allowing us to use $(6.7)$ for $(n-1)$ vectors. This leaves us with the result that the only permutation for which we have a non-vanishing contribution is $\alpha_{\sigma(1)}>\alpha_{\sigma(2)}>\cdots>\alpha_{\sigma(n-1)}$. Combining this with the result that $\alpha_{\sigma(n)}<\alpha_{\sigma(n-1)}$ we see that the only permutation for which the result is non-zero is $\sigma(1,2, \ldots, n)=(n, n-1, \ldots, 1)$. This is the desired result. 


\section{Equivalence of "Higgs" and "Coulomb" branch wall-crossing formulæ}

Manschot et al. [30] also proposed a different prescription for computing $g_{\text {ref }}\left(\alpha_{1}, \ldots, \alpha_{n} ; y\right)$ called the "coulomb branch formula". The formula is similar to $(2.1)$, but with $\mathrm{N}_{\text {higgs }}^{(n)}\left(\left\{\alpha_{i}\right\} ; \sigma\right)$ replaced by an apparently different quantity, which we shall denote by $\mathrm{N}_{\text {coulomb }}^{(n)}\left(\left\{\alpha_{i}\right\} ; \sigma\right)$. The prescription for computing $\mathrm{N}_{\text {coulomb }}^{(n)}\left(\left\{\alpha_{i}\right\} ; \sigma\right)$ associated with a given permutation $\sigma$ is as follows:

(1) Let us define $\widetilde{\alpha}_{i} \equiv \alpha_{\sigma(i)}$ as usual. Now for a given permutation $\sigma$ we consider a function $W$ of $n$ real variables $x_{1}, \ldots, x_{n}$ ordered as $x_{1}<$ $x_{2}<\cdots<x_{n}$ :

$$
W=-\sum_{i<j}\left\langle\widetilde{\alpha}_{i}, \widetilde{\alpha}_{j}\right\rangle \log \left|x_{i}-x_{j}\right|+\Lambda \sum_{i, j=1}^{n}\left\langle\widetilde{\alpha}_{i}, \widetilde{\alpha}_{j}\right\rangle x_{j}
$$

where $\Lambda$ is a positive constant. It will be useful to think of $W$ as the potential for $n$ 1D particles positioned at $x_{1}, \ldots, x_{n} . W$ is invariant under simultaneous translation of all $x_{i}$ 's by a constant.

(2) $\mathrm{N}_{\text {coulomb }}^{(n)}\left(\left\{\alpha_{i}\right\} ; \sigma\right)$ is non vanishing only if there is an extremum of $W$ with respect to the variables $x_{2}, \ldots, x_{n}$ at fixed $x_{1}=0$ :

$$
\frac{\partial W}{\partial x^{i}}=0 \quad \text { for } 2 \leq i \leq n
$$

This corresponds to an equilibrium configuration of the $n$ particles and fixing $x_{1}=0$ (or any other fixed value) is possible due to translation invariance of $W$ mentioned above. When this condition is satisfied $\mathrm{N}_{\text {coulomb }}^{(n)}\left(\left\{\alpha_{i}\right\} ; \sigma\right)$ takes value 1 or -1 , with the sign given by the sign of $\operatorname{det} M$ at the extremum, where $M_{i j}=\left(\partial^{2} W / \partial x_{i} \partial x_{j}\right), i, j=2, \ldots, n$. If there is more than one extremum then we have to sum over these extrema with weight factors $\operatorname{sign}(\operatorname{det} M)$ associated with these different extrema.

Our goal in this section will be to prove the equality of $\mathrm{N}_{\text {higgs }}^{(n)}\left(\left\{\alpha_{i}\right\} ; \sigma\right)$ and $\mathrm{N}_{\text {coulomb }}^{(n)}\left(\left\{\alpha_{i}\right\} ; \sigma\right)$. We first note that the prescription for computing $\mathrm{N}_{\text {coulomb }}^{(n)}$ given above is invariant under small deformations of $\left\{\alpha_{i}\right\}$ under which the extrema change their positions or (dis)appear in pairs by merger or pair creation, but does not (dis)appear singly. The latter may occur if 
during the deformation a nearest neighbor pair $\left(x_{i}, x_{i+1}\right)$ at the extremum approach each other so that beyond the point of merger of $x_{i}$ and $x_{i+1}$ the extremum ceases to exist, or if at the extremum a set of $x_{i}$ 's get separated from the rest by an infinite distance beyond which the extremum ceases to exist. Now since as $x_{i} \rightarrow x_{i+1}$ the dominant term in $\partial W / \partial x_{i}$ is given by

$$
\left\langle\widetilde{\alpha}_{i}, \widetilde{\alpha}_{i+1}\right\rangle\left(x_{i+1}-x_{i}\right)^{-1}
$$

we see that this term cannot be cancelled by the contribution from any other term unless $\left\langle\widetilde{\alpha}_{i}, \widetilde{\alpha}_{i+1}\right\rangle$ approaches zero. Thus as long as the deformations preserve the relative ordering of the $\alpha_{i}$ 's so that for no pair $\left\langle\alpha_{i}, \alpha_{j}\right\rangle$ passes through 0 , we avoid the first possibility. To examine the second possibility let us suppose that at the extremum the subset of points $\left\{x_{i}, k+1 \leq\right.$ $i \leq n\}$ gets separated from the rest $\left\{x_{i}, 1 \leq i \leq k\right\}$ by an infinite distance. Since at the extramum $\partial W / \partial x_{j}$ should vanish for $j \geq 2$, we must have $\sum_{j=k+1}^{n} \partial W / \partial x_{j}=0$. In the large separation limit the second term in (7.1) dominates and we get

$$
\sum_{j=k+1}^{n} \partial W / \partial x_{j} \simeq \Lambda \sum_{i=1}^{n} \sum_{j=k+1}^{n}\left\langle\widetilde{\alpha}_{i}, \widetilde{\alpha}_{j}\right\rangle=\Lambda\left\langle\bar{\alpha}, \bar{\alpha}-\beta_{k}\right\rangle=-\Lambda\left\langle\bar{\alpha}, \beta_{k}\right\rangle
$$

where $\beta_{k}=\sum_{j=1}^{k} \widetilde{\alpha}_{j}$ and $\bar{\alpha}=\left(\alpha_{1}+\cdots+\alpha_{n}\right)$ as before. Thus as long as this is kept away from zero the extremum of $W$ cannot approach a configuration where $x_{1}, \ldots, x_{k}$ separates by an infinite distance from the rest of the $x_{i}$ 's. Combining these results we come to the conclusion that if we deform the $\alpha_{i}$ 's without changing the relative orientation between the $\alpha_{i}$ 's and the relative orientation between any of the $\beta_{k}$ 's and $\bar{\alpha}, \mathrm{N}_{\text {coulomb }}^{(n)}\left(\left\{\alpha_{i}\right\} ; \sigma\right)$ will remain unchanged. These are the same set of deformations under which $\mathrm{N}_{\text {higgs }}^{(n)}\left(\left\{\alpha_{i}\right\} ; \sigma\right)$ remains unchanged.

We are now ready to begin proving the equality of $\mathrm{N}_{\text {higgs }}^{(n)}$ and $\mathrm{N}_{\text {coulomb }}^{(n)}$. This has been checked explicitly for low values on $n$ in [30]. We shall use the method of induction i.e., assume that the equality of the $\mathrm{N}_{\text {higgs }}^{(m)}$ and $\mathrm{N}_{\text {coulomb }}^{(m)}$ holds for $m \leq n-1$ and then prove the equality of $\mathrm{N}_{\text {higgs }}^{(n)}$ and $\mathrm{N}_{\text {coulomb }}^{(n)}$. Let us consider a particular $\sigma$ and the associated $\widetilde{\alpha}_{i}$ 's. We now deform the pair of charges $\left(\widetilde{\alpha}_{n-1}, \widetilde{\alpha}_{n}\right)$ according to the prescription of Section 5.2: $\widetilde{\alpha}_{n-1} \rightarrow(1+\lambda) \widetilde{\alpha}_{n-1}, \widetilde{\alpha}_{n} \rightarrow\left(1+\lambda^{\prime}\right) \widetilde{\alpha}_{n}$ with $\lambda \alpha_{n-1}+\lambda^{\prime} \alpha_{n} \propto \bar{\alpha}$ and $\lambda<0$. We can increase $|\lambda|$ without changing the relative orientations between the $\alpha_{i}$ 's and between the $\beta_{k}$ 's and $\bar{\alpha}$ till we encounter one of the following situations: either $\widetilde{\alpha}_{n}$ becomes almost zero (which is equivalent to $\beta_{n-1}$ becoming almost parallel to $\bar{\alpha}$ ) or $\widetilde{\alpha}_{n-1}$ becomes almost zero. In either case the 
charge that becomes almost zero does not affect the equilibrium arrangement of the rest of the $(n-1)$ centers obtained by extremizing $W$ with respect to the $(n-2)$ variables. Thus the arrangement of the rest of the centers at the extremum must follow the rules of $\mathrm{N}_{\text {coulomb }}^{(n-1)}\left(\left\{\alpha_{i}\right\} ; \sigma\right)$, which by our ansatz are the same as those of $\mathrm{N}_{\text {higgs }}^{(n-1)}\left(\left\{\alpha_{i}\right\} ; \sigma\right)$. We shall now consider the two possibilities separately.

First suppose that $\widetilde{\alpha}_{n}$ becomes almost zero. In this case, the charges $\widetilde{\alpha}_{1}, \ldots, \widetilde{\alpha}_{n-1}$ for which $\mathrm{N}_{\text {higgs }}^{(n-1)}$ is non-zero can be found by replacing $n+1$ by $n-1$ in $(5.37)$

$$
\beta_{k}\left\{\begin{array}{ll}
<\bar{\alpha} & \text { for } \widetilde{\alpha}_{k+1}>\widetilde{\alpha}_{k}, \\
>\bar{\alpha} & \text { for } \widetilde{\alpha}_{k+1}<\widetilde{\alpha}_{k},
\end{array} \quad \text { for } 1 \leq k \leq n-2 .\right.
$$

By the assumed equality of $N_{\text {higgs }}^{(n-1)}$ and $N_{\text {coulomb }}^{(n-1)}$, we have an equilibrium configuration of $x_{1}, \ldots, x_{n-1}$ iff the charges satisfy (7.5). Since the addition of an infinitesimal charge $\widetilde{\alpha}_{n}$ at $x_{n}$ will not disturb the equilibrium configuration of the other charges, we only need to look for an equilibrium position of $x_{n}$, i.e., an $x_{n}$ satisfying $\partial W / \partial x_{n}=0$. From (7.1) we see that

$$
\begin{aligned}
& W \rightarrow \Lambda \sum_{i=1}^{n-1}\left\langle\widetilde{\alpha}_{i}, \widetilde{\alpha}_{n}\right\rangle x_{n}=\Lambda\left\langle\beta_{n-1}, \bar{\alpha}-\beta_{n-1}\right\rangle x_{n}=\Lambda\left\langle\beta_{n-1}, \bar{\alpha}\right\rangle x_{n} \quad \text { as } x_{n} \rightarrow \infty \\
& W \rightarrow-\left\langle\widetilde{\alpha}_{n-1}, \widetilde{\alpha}_{n}\right\rangle \ln \left|x_{n-1}-x_{n}\right| \quad \text { as } x_{n} \rightarrow x_{n-1} .
\end{aligned}
$$

At both limits the magnitude of $W$ goes to infinity. Thus if these two limits have the same sign then $\partial W / \partial x_{n}$ must vanish somewhere in the range $x_{n-1}<x_{n}<\infty$ and we are guaranteed to have an extremum. This gives

$$
\begin{array}{ll}
\widetilde{\alpha}_{n-1}>\widetilde{\alpha}_{n} & \text { if } \beta_{n-1}>\bar{\alpha}, \\
\widetilde{\alpha}_{n-1}<\widetilde{\alpha}_{n} & \text { if } \beta_{n-1}<\bar{\alpha} .
\end{array}
$$

If this condition is satisfied then we may have more than one solution, but the number of solutions is always odd and all except one contribution cancels when we weigh it by the sign of $\operatorname{det} M$. If (7.7) does not hold then we could have even number of solutions but their contribution will cancel pairwise.

(7.7) precisely extends (7.5) all the way to $k=n-1$. Thus we see that the condition for $\mathrm{N}_{\text {coulomb }}^{(n)}\left(\left\{\alpha_{i}\right\} ; \sigma\right)$ to be non-vanishing coincides with the condition for $\mathrm{N}_{\text {higgs }}^{(n)}\left(\left\{\alpha_{i}\right\} ; \sigma\right)$ to be non-vanishing. We shall now show that the signs of $\mathrm{N}_{\text {coulomb }}^{(n)}\left(\left\{\alpha_{i}\right\} ; \sigma\right)$ and $\mathrm{N}_{\text {higgs }}^{(n)}\left(\left\{\alpha_{i}\right\} ; \sigma\right)$ also agree in this case. In the 
limit when the charge of the $n$th center is small we can ignore the off diagonal $M_{n i}$ and $M_{i n}$ components of the matrix $M$ and express det $M$ as the product of the determinant of the first $(n-2) \times(n-2)$ block and $\partial^{2} W / \partial x_{n}^{2}$. The assumed equality of $\mathrm{N}_{\text {coulomb }}^{(n-1)}\left(\left\{\alpha_{i}\right\} ; \sigma\right)$ and $\mathrm{N}_{\text {higgs }}^{(n-1)}\left(\left\{\alpha_{i}\right\} ; \sigma\right)$ tell us that the sign of the contribution from the first $(n-2) \times(n-2)$ block to $\operatorname{det} M$ is given by $(2.1)$

$$
(-1)^{\sum_{k=1}^{n-2} \Theta\left(\widetilde{\alpha}_{i}, \widetilde{\alpha}_{i+1}\right)}
$$

Now we see from $(7.6),(7.7)$ that if $\widetilde{\alpha}_{n-1}>\widetilde{\alpha}_{n}$ i.e., $\left\langle\widetilde{\alpha}_{n-1}, \widetilde{\alpha}_{n}\right\rangle<0$, then $W \rightarrow-\infty$ as $x_{n} \rightarrow x_{n-1}, \infty$ and hence $\partial^{2} W / \partial x_{n}^{2}$ at the extremum is negative. On the other hand if $\widetilde{\alpha}_{n-1}<\widetilde{\alpha}_{n}$ then $\partial^{2} W / \partial x_{n}^{2}$ at the extremum is positive. Thus $\mathrm{N}_{\text {coulomb }}^{(n)}\left(\left\{\alpha_{i}\right\} ; \sigma\right)$ is obtained by multiplying (7.8) by a factor of $(-1)^{\Theta\left(\widetilde{\alpha}_{n-1}, \widetilde{\alpha}_{n}\right)}$. This reproduces the formula for $\mathrm{N}_{\text {higgs }}^{(n)}$ given in (2.1), showing the equality of $\mathrm{N}_{\text {higgs }}^{(n)}$ and $\mathrm{N}_{\text {coulomb }}^{(n)}$.

Next we consider the case when $\widetilde{\alpha}_{n-1}$ becomes almost zero at the end of the deformation. In this case the arrangement of the charges $\left(\widetilde{\alpha}_{1}, \ldots, \widetilde{\alpha}_{n-2}\right.$, $\left.\widetilde{\alpha}_{n}\right)$ follows the corresponding rules for $\mathrm{N}_{\text {higgs }}^{(n-1)}$. Since $\widetilde{\alpha}_{n-1}$ is almost zero we have $\beta_{n-1} \simeq \beta_{n-2}$, and thus they must be on the same side of $\bar{\alpha}$. The condition on the charges $\widetilde{\alpha}_{1}, \ldots, \widetilde{\alpha}_{n-2}, \widetilde{\alpha}_{n}$ for which $\mathrm{N}_{\text {higgs }}^{(n-1)}$ is non-zero can now be written as

$$
\begin{aligned}
& \beta_{k} \begin{cases}<\bar{\alpha} & \text { for } \widetilde{\alpha}_{k+1}>\widetilde{\alpha}_{k}, \quad \text { for } 1 \leq k \leq n-3, \\
>\bar{\alpha} & \text { for } \widetilde{\alpha}_{k+1}<\widetilde{\alpha}_{k},\end{cases} \\
& \beta_{n-2}, \beta_{n-1} \begin{cases}<\bar{\alpha} & \text { for } \widetilde{\alpha}_{n}>\widetilde{\alpha}_{n-2}, \\
>\bar{\alpha} & \text { for } \widetilde{\alpha}_{n}<\widetilde{\alpha}_{n-2} .\end{cases}
\end{aligned}
$$

By examining the behavior of $W$ as $x_{n-1} \rightarrow x_{n-2}, x_{n}$ we get

$$
\begin{aligned}
& W \rightarrow-\left\langle\widetilde{\alpha}_{n-2}, \widetilde{\alpha}_{n-1}\right\rangle \ln \left|x_{n-2}-x_{n-1}\right| \quad \text { as } x_{n-1} \rightarrow x_{n} \\
& W \rightarrow-\left\langle\widetilde{\alpha}_{n-1}, \widetilde{\alpha}_{n}\right\rangle \ln \left|x_{n-1}-x_{n}\right| \quad \text { as } x_{n-1} \rightarrow x_{n}
\end{aligned}
$$

Thus, in order that they have the same sign so that we have an extremum we need

$$
\widetilde{\alpha}_{n-2}<\widetilde{\alpha}_{n-1}<\widetilde{\alpha}_{n} \quad \text { or } \quad \widetilde{\alpha}_{n-2}>\widetilde{\alpha}_{n-1}>\widetilde{\alpha}_{n}
$$


Combining this with (7.5) we arrive at the result:

$$
\begin{aligned}
& \beta_{k}\left\{\begin{array}{l}
<\bar{\alpha} \text { for } \widetilde{\alpha}_{k+1}>\widetilde{\alpha}_{k}, \\
>\bar{\alpha} \quad \text { for } \widetilde{\alpha}_{k+1}<\widetilde{\alpha}_{k},
\end{array} \text { for } 1 \leq k \leq n-1,\right. \\
& \beta_{n-2}, \beta_{n-1}>\bar{\alpha} \quad \text { or } \quad \beta_{n-2}, \beta_{n-1}<\bar{\alpha} .
\end{aligned}
$$

The second condition is of course needed to have an almost zero $\widetilde{\alpha}_{n-1}$ in the first place. The first condition is the same as the one for getting a nonvanishing contribution to $\mathrm{N}_{\text {higgs }}^{(n)}\left(\left\{\alpha_{i}\right\} ; \sigma\right)$. Thus we see that the requirement for having non-vanishing $\mathrm{N}_{\text {coulomb }}^{(n)}\left(\left\{\alpha_{i}\right\} ; \sigma\right)$ again reduces to that of having non-vanishing $\mathrm{N}_{\text {higgs }}^{(n)}\left(\left\{\alpha_{i}\right\} ; \sigma\right)$.

We now need to calculate the sign of the contribution. The centers $1, \ldots, n-2, n$ give a factor of $(-1)^{\sum_{k=1}^{n-3} \Theta\left(\widetilde{\alpha}_{i}, \widetilde{\alpha}_{i+1}\right)}(-1)^{\Theta\left(\widetilde{\alpha}_{n-2}, \widetilde{\alpha}_{n}\right)}$. Using (7.11) the last factor may be written as $(-1)^{\Theta\left(\widetilde{\alpha}_{n-2}, \widetilde{\alpha}_{n-1}\right)}$. By studying the behavior of $W$ as $x_{n-1} \rightarrow x_{n-2}, x_{n}$ we see that the extra contribution from the sign of $\partial^{2} W / \partial x_{n-1}^{2}$ is positive for $\widetilde{\alpha}_{n-2}<\widetilde{\alpha}_{n-1}<\widetilde{\alpha}_{n}$ and negative for $\widetilde{\alpha}_{n-2}>$ $\widetilde{\alpha}_{n-1}>\widetilde{\alpha}_{n}$. Thus, the extra contribution can be written as $(-1)^{\Theta\left(\widetilde{\alpha}_{n-1}, \widetilde{\alpha}_{n}\right)}$. Combining these factors we get

$$
\mathrm{N}_{\text {coulomb }}^{(n)}\left(\left\{\alpha_{i}\right\} ; \sigma\right)=(-1)^{\sum_{i=1}^{n-1} \Theta\left(\widetilde{\alpha}_{i}, \widetilde{\alpha}_{i+1}\right)}
$$

in agreement with formula (2.1) for $\mathrm{N}_{\text {higgs }}^{(n)}\left(\left\{\alpha_{i}\right\} ; \sigma\right)$. This establishes the equality of $\mathrm{N}_{\text {higgs }}^{(n)}\left(\left\{\alpha_{i}\right\} ; \sigma\right)$ and $\mathrm{N}_{\text {coulomb }}^{(n)}\left(\left\{\alpha_{i}\right\} ; \sigma\right)$.

\section{Acknowledgment}

I wish to thank J. Manschot and B. Pioline for useful correspondence. This work was supported in part by the J. C. Bose fellowship of the Department of Science and Technology, India and the project 11-R\&D-HRI-5.02-0304.

\section{Appendix A. Physical interpretation of $g_{\text {higgs }}$}

In this appendix, we shall show the equivalence between (2.1) and the formula given in [30]. Let $\{\sigma(1), \sigma(2), \ldots, \sigma(n)\}$ denote a permutation of $\{1, \ldots, n\}$. Associated with each such permutation we can associate a unique number $s$ and a set of numbers $m_{1}, \ldots, m_{s-1}$ by imposing the following requirements:

(1) $1<m_{1}<m_{2}<\cdots m_{s-1} \leq n$. 
(2) $\sigma(m)>\sigma(m-1)$ for $m \neq m_{1}, m_{2}, \ldots m_{s-1}$.

(3) $\sigma\left(m_{a}\right)<\sigma\left(m_{a}-1\right)$ for $1 \leq a \leq s-1$.

Physically this partitions the ordered set $\{\sigma(1), \sigma(2), \ldots, \sigma(n)\}$ into $s$ maximally increasing subsequences: the $\sigma(i)$ 's increase monotonically with $i$ for $i$ between 1 and $m_{1}-1$, between $m_{a}$ and $m_{a+1}-1$ for $1 \leq a \leq(s-2)$, and between $m_{s-1}$ and $n$, but between $m_{a}-1$ and $m_{a} \forall a$ the monotone increase is broken. The expression for $\mathrm{N}_{\text {higgs }}^{(n)}\left(\left\{\alpha_{i}\right\} ; \sigma\right)$ given in $(2.1)$ can now be rewritten as

$$
\text { (A.1) } \begin{aligned}
\mathrm{N}_{\text {higgs }}^{(n)}\left(\left\{\alpha_{i}\right\} ; \sigma\right)= & (-1)^{s-1} \prod_{\substack{k=m_{1}, \ldots m_{s-1} \\
\text { ( }}} \Theta\left(\alpha_{1}+\cdots+\alpha_{n}, \sum_{i=k}^{n} \alpha_{\sigma(i)}\right) \\
& \times \prod_{\substack{k=2 \\
k \neq m_{1}, \ldots m_{s-1}}}^{n} \Theta\left(\sum_{i=k}^{n} \alpha_{\sigma(i)}, \alpha_{1}+\cdots+\alpha_{n}\right)
\end{aligned}
$$

Let us define a set of vectors $\beta^{(1)}, \ldots \beta^{(s)}$ as follows:

$$
\begin{aligned}
& \beta^{(1)}=\sum_{i=m_{s-1}}^{n} \alpha_{\sigma(i)}, \quad \beta^{(a)}=\sum_{i=m_{s-a}}^{m_{s-a+1}-1} \alpha_{\sigma(i)} \quad \text { for } 2 \leq a \leq s-1, \\
& \beta^{(s)}=\sum_{i=1}^{m_{1}-1} \alpha_{\sigma(i)} .
\end{aligned}
$$

This allows us to associate to every permutation $\sigma(i)$ a unique set of vectors $\left\{\beta^{(a)}\right\}$. For example, if for $n=4$ we consider the permutation (2134) then the partition of (2134) containing maximally increasing subsequences are $\{\{2\},\{1,3,4\}\}$, giving $\beta^{(1)}=\alpha_{1}+\alpha_{3}+\alpha_{4}, \beta^{(2)}=\alpha_{2}$. The product of the $\Theta$ 's in (2.1) restricts the sum over permutations to a set $K$ of permutations satisfying the following conditions:

(1) The first set of $\Theta$ functions in (A.1) ensure that the vectors $\left\{\beta^{(a)}\right\}$ associated with the permutation $\sigma$ should satisfy

$$
\left\langle\sum_{a=1}^{b} \beta^{(a)}, \alpha_{1}+\cdots+\alpha_{n}\right\rangle>0 \quad \forall \quad b \text { with } 1 \leq b \leq s-1
$$

(2) We can associate with the permutation $\sigma$ many other partitions containing increasing subsequences which are not maximal, by dropping the 
third condition $\sigma\left(m_{a}\right)<\sigma\left(m_{a}-1\right)$. Thus, for example, for the permutation (2134) discussed above, examples of partitions containing nonmaximal increasing subsequences are $\{\{2\},\{1\},\{3\},\{4\}\},\{\{2\},\{1,3\}$, $\{4\}\}$ and $\{\{2\},\{1\},\{3,4\}\}$. The second set of $\Theta$-functions in (A.1) guarantee that if we construct the $\beta^{(a)}$ 's for any such partition following the same procedure, then the condition (A.3) must fail for at least one $b$.

Once we have identified the set $K$ of permutations satisfying these properties, (2.1) reduces to:

$$
\begin{aligned}
g_{\text {higgs }}\left(\alpha_{1}, \ldots, \alpha_{n}\right)= & (-y)^{-1+n-\sum_{i<j} \alpha_{i j}}\left(y^{2}-1\right)^{1-n} \\
& \times \sum_{\sigma \in K}(-1)^{s-1}(-y)^{2 \sum_{\substack{l<k \\
\sigma(l)<\sigma(k)}} \alpha_{\sigma(l) \sigma(k)}} .
\end{aligned}
$$

This is the formula for $g_{\text {higgs }}$ derived in Section 3.3 of [30]. ${ }^{7}$ The original Reineke formula [45] corresponds to summing over many more terms corresponding to all increasing sequences (i.e., not just the maximal increasing sequences), but it was shown in [30] that the contribution from many of these terms cancel and at the end only the contribution from the terms given in (A.4), corresponding to maximal increasing sequences, survive.

\section{Appendix B. Proof of the $\Theta$ identity}

In this appendix we shall prove the identity (5.50). Let us denote the lefthand side of (5.50) by $P(N, c)$, i.e.,

$$
\begin{aligned}
P(N, c) \equiv & \prod_{i=1}^{N-1}[\Theta(f(i+1)-c) \Theta(f(i+1)-f(i))-\Theta(c-f(i+1)) \\
& \times \Theta(f(i)-f(i+1))]+\sum_{k=1}^{N-1} \sum_{I_{1}=2}^{N} \sum_{I_{2}=I_{1}+1}^{N} \sum_{I_{3}=I_{2}+1}^{N} \ldots \sum_{I_{k}=I_{k-1}+1}^{N}
\end{aligned}
$$

\footnotetext{
${ }^{7}$ In the last term [30] had $y^{\cdots}$ instead of $(-y)^{\cdots}$. For physical $\alpha_{i j}$ 's which are integers the two formulæ give identical results since the exponent is an even integer.
} 


$$
\begin{aligned}
& \times\left\{\Theta\left(c-f\left(I_{1}\right)\right) \prod_{\ell=1}^{k-1} \Theta\left(f\left(I_{\ell}\right)-f\left(I_{\ell+1}\right)\right) \prod_{i=1}^{I_{1}-2}[\Theta(f(i+1)-c)\right. \\
& \times \Theta(f(i+1)-f(i))-\Theta(c-f(i+1)) \Theta(f(i)-f(i+1))] \\
& \times \prod_{\ell=1}^{k-1} \prod_{i=I_{\ell}}^{I_{\ell+1}-2}\left[\Theta\left(f(i+1)-f\left(I_{\ell}\right)\right) \Theta(f(i+1)-f(i))\right. \\
& \left.-\Theta\left(f\left(I_{\ell}\right)-f(i+1)\right) \Theta(f(i)-f(i+1))\right] \\
& \times \prod_{i=I_{k}}^{N-1}\left[\Theta\left(f(i+1)-f\left(I_{k}\right)\right) \Theta(f(i+1)-f(i))\right. \\
& \left.\left.-\Theta\left(f\left(I_{k}\right)-f(i+1)\right) \Theta(f(i)-f(i+1))\right]\right\} .
\end{aligned}
$$

We shall assume that (5.50) is valid up to a certain value of $N$, e.g., we have

$$
P(M, c)=\prod_{i=1}^{M-1} \Theta(f(i+1)-f(i)), \quad \text { for } M \leq N-1
$$

and then show that (B.2) also holds for $M=N$. Now in the $k \geq 1$ terms in (B.1) the sum over $k$ and $I_{2}, \ldots, I_{k}$ for fixed $I_{1}$, after factoring out the $I_{1}$ dependent but $k$ independent terms, has the same structure as $P\left(N-I_{1}+\right.$ $\left.1, f\left(I_{1}\right)\right)$ with $f(i) \rightarrow f\left(i+I_{1}-1\right)$. This gives

$$
\begin{aligned}
P(N, c)= & \prod_{i=1}^{N-1}[\Theta(f(i+1)-c) \Theta(f(i+1)-f(i)) \\
& -\Theta(c-f(i+1)) \Theta(f(i)-f(i+1))] \\
& +\left.\sum_{I_{1}=2}^{N} \Theta\left(c-f\left(I_{1}\right)\right) P\left(N-I_{1}+1, f\left(I_{1}\right)\right)\right|_{f(i) \rightarrow f\left(i+I_{1}-1\right)} \\
& \times \prod_{i=1}^{I_{1}-2}[\Theta(f(i+1)-c) \Theta(f(i+1)-f(i))-\Theta(c-f(i+1)) \\
& \times \Theta(f(i)-f(i+1))] .
\end{aligned}
$$


Using (B.2) the result can be expressed as

$$
\begin{aligned}
P(N, c)= & \prod_{i=1}^{N-1}[\Theta(f(i+1)-c) \Theta(f(i+1)-f(i))-\Theta(c-f(i+1)) \\
& \times \Theta(f(i)-f(i+1))]+\sum_{I_{1}=2}^{N} \Theta\left(c-f\left(I_{1}\right)\right) \prod_{i=I_{1}}^{N-1} \Theta(f(i+1)-f(i)) \\
& \times \prod_{i=1}^{I_{1}-2}[\Theta(f(i+1)-c) \Theta(f(i+1)-f(i)) \\
& -\Theta(c-f(i+1)) \Theta(f(i)-f(i+1))] .
\end{aligned}
$$

Using the relation

$$
\begin{aligned}
\Theta(x) \Theta(y)-\Theta(-x) \Theta(-y) & =\Theta(x)(1-\Theta(-y))-(1-\Theta(x)) \Theta(-y) \\
& =\Theta(x)-\Theta(-y)
\end{aligned}
$$

we can simplify (B.4) to

$$
\begin{aligned}
P(N, c)= & \prod_{i=1}^{N-1}[\Theta(f(i+1)-f(i))-\Theta(c-f(i+1))] \\
& +\sum_{I_{1}=2}^{N} \Theta\left(c-f\left(I_{1}\right)\right) \prod_{i=I_{1}}^{N-1} \Theta(f(i+1)-f(i)) \\
& \times \prod_{i=1}^{I_{1}-2}[\Theta(f(i+1)-f(i))-\Theta(c-f(i+1))] .
\end{aligned}
$$

We can manipulate this by separating out the $I_{1}=N$ term in the sum and combining it with the first term. This gives

$$
\begin{aligned}
P(N, c)= & \prod_{i=1}^{N-2}[\Theta(f(i+1)-f(i))-\Theta(c-f(i+1))] \\
& \times[\Theta(f(N)-f(N-1))-\Theta(c-f(N))+\Theta(c-f(N))] \\
& +\sum_{I_{1}=2}^{N-1} \Theta\left(c-f\left(I_{1}\right)\right) \prod_{i=I_{1}}^{N-1} \Theta(f(i+1)-f(i))
\end{aligned}
$$




$$
\begin{aligned}
& \times \prod_{i=1}^{I_{1}-2}[\Theta(f(i+1)-f(i))-\Theta(c-f(i+1)] \\
= & \Theta(f(N)-f(N-1))\left\{\prod_{i=1}^{N-2}[\Theta(f(i+1)-f(i))-\Theta(c-f(i+1))]\right. \\
& +\sum_{I_{1}=2}^{N-1} \Theta\left(c-f\left(I_{1}\right)\right) \prod_{i=I_{1}}^{N-2} \Theta(f(i+1)-f(i)) \\
& \left.\times \prod_{i=1}^{I_{1}-2}[\Theta(f(i+1)-f(i))-\Theta(c-f(i+1))]\right\} .
\end{aligned}
$$

We now note that the term inside the \{\} has the same form as the righthand side of (B.6) with $N$ replaced by $N-1$. Thus, we can manipulate it again in the same way, pulling out a factor of $\Theta(f(N-1)-f(N-2))$ and replacing $N$ by $N-1$ again in the remaining factor. Repeating this process we arrive at the result:

$$
P(N, c)=\prod_{i=1}^{N-1} \Theta(f(i+1)-f(i))
$$

which is the desired result.

\section{References}

[1] S. Cecotti and C. Vafa, On classification of $N=2$ supersymmetric theories, Commun. Math. Phys. 158 (1993), 569-644, [arXiv:hep-th/9211097].

[2] N. Seiberg and E. Witten, Electric - magnetic duality, monopole condensation, and confinement in $N=2$ supersymmetric Yang-Mills theory, Nucl. Phys. B 426 (1994), 19-52, [arXiv:hep-th/9407087].

[3] N. Seiberg and E. Witten Monopoles, duality and chiral symmetry breaking in $N=2$ supersymmetric QCD, Nucl. Phys. B 431 (1994), 484-550, [arXiv:hep-th/9408099].

[4] F. Ferrari and A. Bilal, The strong coupling spectrum of the Seiberg-Witten theory, Nucl. Phys. B 469 (1996), 387-402, [arXiv:hep-th/9602082]. 
[5] A. Bilal and F. Ferrari, Curves of marginal stability, and weak and strong coupling BPS spectra in $N=2$ supersymmetric QCD, Nucl. Phys. B 480 (1996), 589-622, [arXiv:hep-th/9605101].

[6] M.R. Douglas, D-branes, categories and $N=1$ supersymmetry, J. Math. Phys. 42 (2001), 2818-2843, [arXiv:hep-th/0011017].

[7] F. Denef, Supergravity flows and D-brane stability, J. High Energy Phys. 08 (2000) 050, [arXiv:hep-th/0005049].

[8] F. Denef, Quantum quivers and hall/hole halos, J. High Energy Phys. 10 (2002) 023, [arXiv:hep-th/0206072 [arXiv:hep-th]].

[9] B. Bates and F. Denef, Exact solutions for supersymmetric stationary black hole composites, [arXiv:hep-th/0304094].

[10] F. Denef and G.W. Moore, Split states, entropy enigmas, holes and halos, [arXiv:hep-th/0702146 [HEP-TH]].

[11] M. Kontsevich and Y. Soibelman, Stability structures, motivic Donaldson-Thomas invariants and cluster transformations, [arXiv:0811.2435 [math.AG]].

[12] M. Kontsevich and Y. Soibelman, Motivic Donaldson-Thomas invariants: summary of results, [arXiv:0910.4315 [math.AG]].

[13] M. Kontsevich and Y. Soibelman, Cohomological hall algebra, exponential hodge structures and motivic Donaldson-Thomas invariants, [arXiv:1006.2706 [math.AG]].

[14] D. Joyce, Configurations in abelian categories. IV. Invariants and changing stability conditions, Adv. Math. 217(1) (2008), 125-204, [arXiv:math/0410268].

[15] D. Joyce and Y. Song, A Theory of generalized Donaldson-Thomas invariants, [arXiv:0810.5645 [math.AG]].

[16] D. Joyce, Generalized Donaldson-Thomas invariants, [arXiv:0910. 0105 [math.AG]].

[17] E. Diaconescu and G.W. Moore, Crossing the wall: Branes versus bundles, [arXiv:0706.3193 [arXiv:hep-th]].

[18] D. Gaiotto, G.W. Moore and A. Neitzke, Four-dimensional wallcrossing via three-dimensional field theory, Commun. Math. Phys. 299 (2010), 163-224, [arXiv:0807.4723 [arXiv:hep-th]]. 
[19] S. Alexandrov, B. Pioline, F. Saueressig and S. Vandoren, D-instantons and twistors, J. High Energy Phys. 03 (2009) 044, [arXiv:0812.4219 [arXiv:hep-th]].

[20] J. de Boer, S. El-Showk, I. Messamah and D. Van den Bleeken, Quantizing $N=2$ Multicenter Solutions, J. High Energy Phys. 05 (2009) $002,0807.4556$.

[21] D.L. Jafferis and G.W. Moore, Wall crossing in local Calabi Yau manifolds, [arXiv:0810.4909 [arXiv:hep-th]].

[22] S. Cecotti and C. Vafa, BPS wall crossing and topological strings, [arXiv:0910.2615 [hep-th]].

[23] J. Stoppa, D0-D6 states counting and $G W$ invariants, arXiv: 0912.2923.

[24] Y. Toda, On a computation of rank two Donaldson-Thomas invariants, arXiv:0912.2507.

[25] W.-Y. Chuang, D.-E. Diaconescu and G. Pan, Rank two ADHM invariants and Wallcrossing, arXiv:1002.0579.

[26] S. Cecotti, A. Neitzke and C. Vafa, R-Twisting and 4d/2d correspondences, [arXiv:1006.3435 [hep-th]].

[27] D. Gaiotto, G.W. Moore and A. Neitzke, Framed BPS States, [arXiv: 1006.0146 [hep-th]].

[28] E. Andriyash, F. Denef, D.L. Jafferis and G.W. Moore, Wall-crossing from supersymmetric galaxies, [arXiv:1008.0030 [hep-th]].

[29] J. Manschot, Stability and duality in $N=2$ supergravity, Commun. Math. Phys. 299 (2010), 651-676, [arXiv:0906.1767 [arXiv: hep-th]].

[30] J. Manschot, B. Pioline and A. Sen, Wall Crossing from Boltzmann black hole halos, J. High Energy Phys. JHEP 07 (2011) 059, [arXiv: 1011.1258 [hep-th]].

[31] S. Lee and P. Yi, Framed BPS states, moduli dynamics, and WallCrossing, J. High Energy Phys. JHEP 04 (2011) 098, [arXiv: 1102.1729 [hep-th]].

[32] B. Pioline, Four ways across the wall, [arXiv:1103.0261 [hep-th]]. 
[33] J. Manschot, B. Pioline and A. Sen, A Fixed point formula for the index of multi-centered $N=2$ black holes, J. High Energy Phys. 1105 (2011) 057, [arXiv: 1103.1887 [hep-th]].

[34] H. Kim, J. Park, Z. Wang and P. Yi, Ab initio Wall-Crossing, J. High Energy Phys. JHEP 1109 (2011) 079, [arXiv:1107.0723 [hep-th]].

[35] M. Alim, S. Cecotti, C. Cordova, S. Espahbodi, A. Rastogi and C. Vafa, $B P S$ Quivers and spectra of complete $N=2$ quantum field theories, arXiv:1109.4941 [hep-th].

[36] S. Alexandrov, D. Persson, B. Pioline, Wall-crossing, Rogers dilogarithm, and the $Q K / H K$ correspondence, [arXiv:1110.0466 [arXiv: hep-th]].

[37] A. Neitzke, On a hyperholomorphic line bundle over the Coulomb branch, arXiv:1110.1619 [hep-th].

[38] D.V.D. Bleeken, BPS dyons and Hesse flow, arXiv:1111.6979 [hep-th].

[39] J. Stoppa, Joyce-Song wall-crossing as an asymptotic expansion, arXiv:1112.2174 [math.AG].

[40] T. Dimofte and S. Gukov, Refined, motivic, and quantum, Lett. Math. Phys. 91 (2010), 1, arXiv:0904.1420.

[41] T. Dimofte, S. Gukov and Y. Soibelman, Quantum wall crossing in $N=2$ gauge theories, Lett. Math. Phys. 95 (2011), 1-25, arXiv:0912.1346.

[42] J. Manschot, Wall-crossing of D4-branes using flow trees, arXiv: 1003.1570.

[43] J. Manschot, The Betti numbers of the moduli space of stable sheaves of rank 3 on P2, arXiv:1009.1775.

[44] T. Nishinaka, Multiple D4-D2-D0 on the Conifold and Wall-crossing with the Flop, arXiv:1010.6002.

[45] M. Reineke, The Harder-Narasimhan system in quantum groups and cohomology of quiver moduli, Invent. Math. 152(2) (2003), 349-368, [arXiv:math/0204059].

[46] J. Manschot, BPS invariants of semi-stable sheaves on $p^{2}$ and its blowup, arXiv:1109.4861 [math-ph]. 
[47] M. Reineke, Poisson automorphisms and quiver moduli, [arXiv: $0804.3214 \mathrm{v} 2$ [math.RT]].

[48] So Okada, On Euler characteristics for large Kronecker quivers, arXiv:1106.4238v2 [math.AG].

[49] M. Reineke, J. Stoppa and T. Weist, MPS degeneration formula for quiver moduli and refined $G W /$ Kronecker correspondence, arXiv: $1110.4847 v 1$ [math.AG].

Harish-Chandra Research Institute

ChHatnag Road

JHUSI

Allahabad 211019

INDIA

E-mail address: sen@mri.ernet.in

ReCeived January 8, 2012 
